\title{
Region-Based Three-Dimensional Wavelet Transform Coding
}

\author{
submitted by \\ Kaibin Wang, B.Eng., M.Eng. \\ A thesis submitted to \\ the Faculty of Graduate Studies and Research \\ in partial fulfillment of \\ the requirements for the degree of \\ Master of Applied Science
}

Ottawa-Carleton Institute for Electrical and Computer Engineering
Department of Systems and Computer Engineering
Carleton University
Ottawa, Canada

May, 2005

(C) Kaibin Wang, 2005, 
Library and

Archives Canada

Published Heritage

Branch

395 Wellington Street

Ottawa ON K1A ON4

Canada
Bibliothèque et

Archives Canada

0-494-06805-1

Direction du

Patrimoine de l'édition

395, rue Wellington

Ottawa ON K1A ON4

Canada

\author{
You the Votre reference \\ ISBN. \\ Our file Notre reference \\ ISBN
}

NOTICE:

The author has granted a nonexclusive license allowing Library and Archives Canada to reproduce, publish, archive, preserve, conserve, communicate to the public by telecommunication or on the Internet, loan, distribute and sell theses worldwide, for commercial or noncommercial purposes, in microform. paper, electronic and/or any other formats.

The author retains copyright ownership and moral rights in this thesis. Neither the thesis nor substantial extracts from it may be printed or otherwise reproduced without the author's permission.
AVIS:

L'auteur a accordé une licence non exclusive permettant à la Bibliothèque et Archives Canada de reproduire, publier, archiver, sauvegarder, conserver, transmettre au public par télécommunication ou par l'Internet, prêter. distribuer et vendre des thèses partout dans le monde, à des fins commerciales ou autres, sur support microforme. papier, électronique et/ou autres formats.

L'auteur conserve la propriété du droit d'auteur et des droits moraux qui protège cette thèse. $\mathrm{Ni}$ la thèse ni des extraits substantiels de celle-ci ne doivent être imprimés ou autrement reproduits sans son autorisation.
In compliance with the Canadian

Privacy Act some supporting forms may have been removed from this thesis.

While these forms may be included in the document page count, their removal does not represent any loss of content from the thesis.
Conformément à la loi canadienne sur la protection de la vie privée, quelques formulaires secondaires ont été enlevés de cette thèse.

Bien que ces formulaires aient inclus dans la pagination, il n'y aura aucun contenu manquant. 
The undersigned recommend to the Faculty of Graduate Studies and Research acceptance of the thesis

\title{
Region-Based Three-Dimensional Wavelet Transform Coding
}

\author{
submitled by \\ Kaibin Wang, B.Eng., M.Eng. \\ in partial fulfillment of the requirements for \\ the degree of Master of Applied Science in Electrical Engineering
}

Thesis Supervisor

Chair, Department of Systems and Computer Engineering

Ottawa-Carleton Institute for Electrical and Computer Engineering

Department of Systems and Computer Engineering

Carleton University

Otlawa. Canada

Miy, 2005

ii 


\section{Abstract}

Region-based coding is an important feature provided in today's image coding schemes including SPIHT and JPEG2000 as it allows different regions of interest in an image to be encoded at different bit rates and hence at different qualities rather than encoding the entire image with a single quality constraint. In this thesis, we consider three-dimensional region-based coding of volumetric medical datasets and video sequences. A 3D SA-DWT is used to decompose the data with multiple, arbitrarily shaped regions to obtain the representation of the regions in the transform domain. Then. a modified 3D SPIHT coding algorithm based on an unbalanced tree structure is adopted in 3D region-based coding. This coding scheme offers good rate-distortion performance with additional features such as distortion scalability and flexibility in precise rate control. Experimental results show that the proposed algorithm outperforms the other coding schemes based on SPIHT algorithm in terms of R-D performance. 


\section{Acknowledgements}

I would like to express my sincere gratitude to my thesis supervisor. Dr. Aysegul Cuhadar, for her excellent guidance, encouragement, patience, and financial support during my study period. Her high academic standards have made the learning process a valuable experience that will benefit me the rest of my life.

I also would like to thank Dr. lan Cameron to provide the MR volumetric datasets to perform our experiments. I want also to thank Geoffrey Green for his kind and generous help during my graduate study.

Finally, I am grateful to my family. Thanks to my parents, for their encouragement and support all through my life. Special thanks to my wife Mingming and my daughter Jiayi, for their love and support all the way along. 


\section{Table of Contents}

Abstract........................................................................................................................... iii

Acknowledgements ............................................................................................ iv

Table of Contents.............................................................................................................v

List of Figures ......................................................................................................... vii

List of Tables ........................................................................................

List of Acronyms................................................................................................................ xii

Chapter 1 Introduction .............................................................................................1

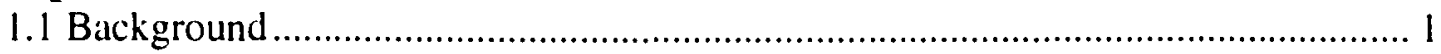

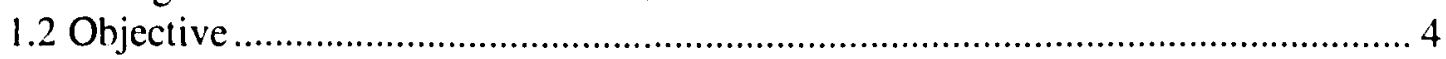

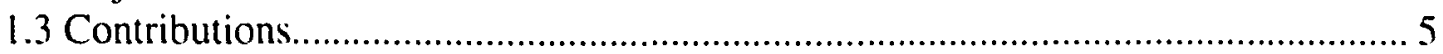

1.4 Thesis Outline ..................................................................................................... 6

Chapter 2 Subband Transform Coding and Wavelet Transform .............8

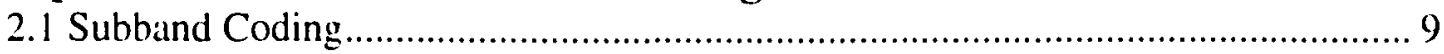

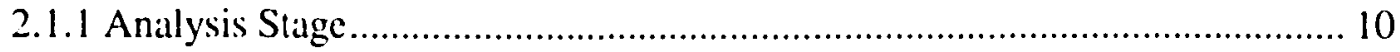

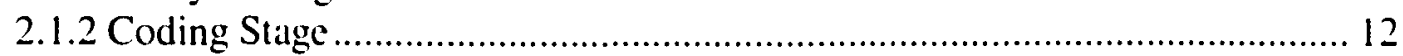

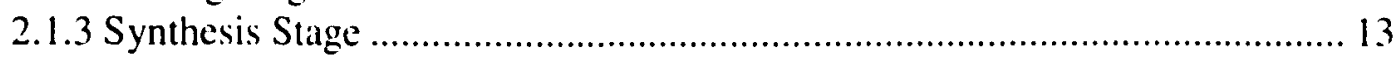

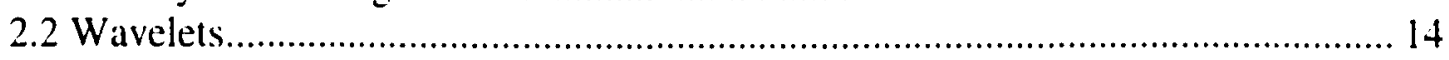

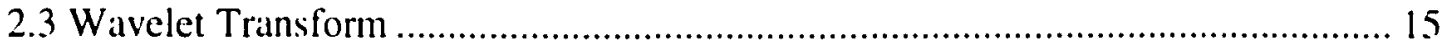

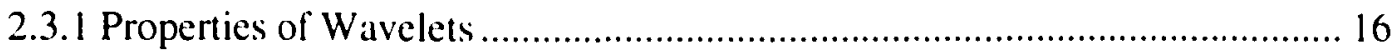

2.3.2 Representation of Spatial Hierarchies...................................................... 18

2.3.3 Representation of Frequency Hierarchies ................................................... 20

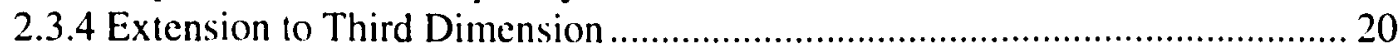

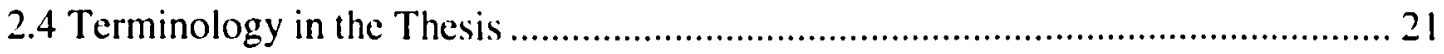

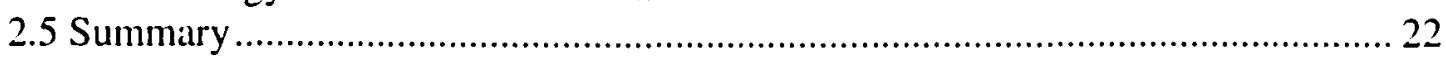

Chapter 3 Shape-Adaptive DWT and ROI Coding .............................. 23

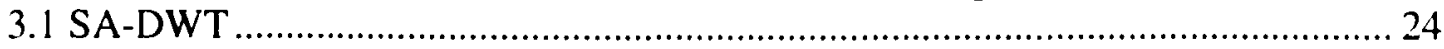

3.1.1 Extension of Finite Length Signals............................................................. 24

3.1.2 Wavelet Representation with Odd-Length Biorthogonal Wavelet Basis ....... 25 


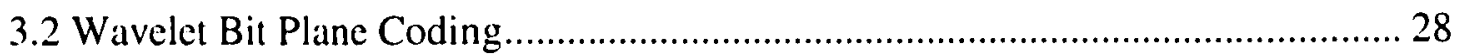

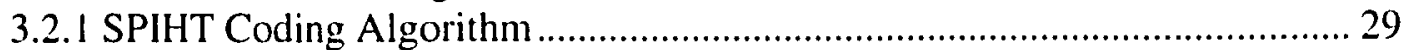

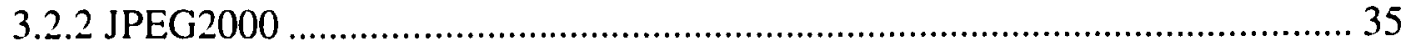

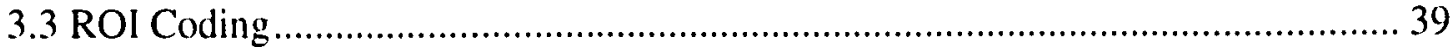

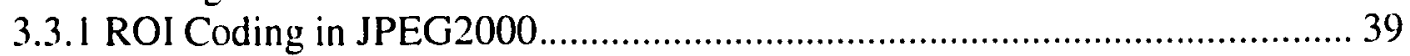

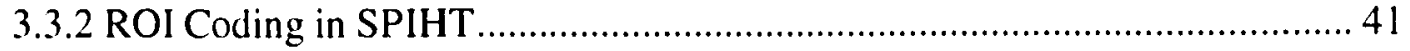

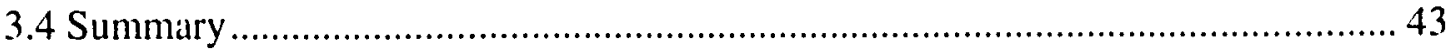

Chapter 4 SPIHT-Based Three-Dimensional Image Coding ................. 45

4.1 Three-Dimensional SPIHT .............................................................................. 46

4.1.1 3D-SPIHT Coding using Dyadic Wavelet Transform ................................... 48

4.1.2 Three-Dimensional SPIHT Coding using Wavelet Packet Transform ............51

4.2 Optimal 3D Coefficient Tree Algorithm............................................................. 54

4.3 Unbalanced Tree Algorithm for 3D ROI Coding ……....................................... 56

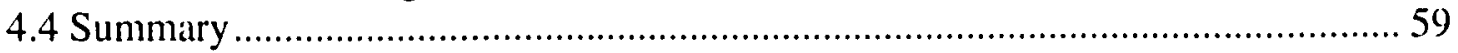

Chapter 5 Three-Dimensional MRMQ Coding ....................................... 61

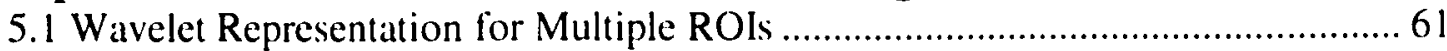

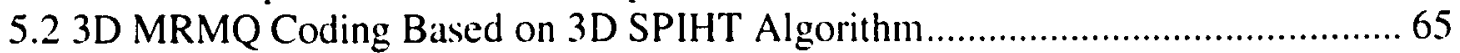

5.2.1 3D MRMQ-SPIHT Algorithm with Sets of Descendant Labels .................... 65

5.2.2 3D MRMQ-SPIHT Algorithm with Unbalanced Tree ................................... 67

5.3 Bit Stream Structure for Multiple ROIs............................................................ 71

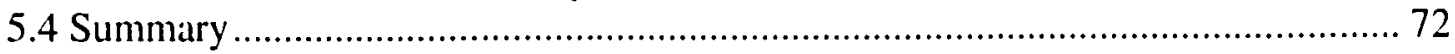

Chapter 6 Experimental Results ........................................................... 73

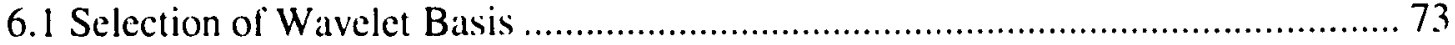

6.2 Compression of Volumetric Medical Datasets .................................................... 74

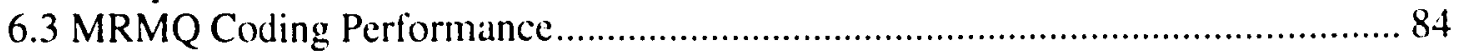

6.4 Compression of Video Sequences ................................................................... 87

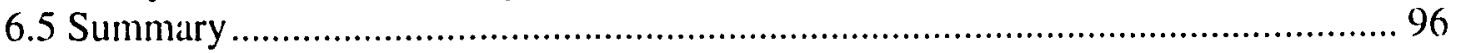

Chapter 7 Conclusions ................................................................................. 98

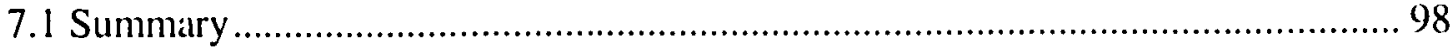

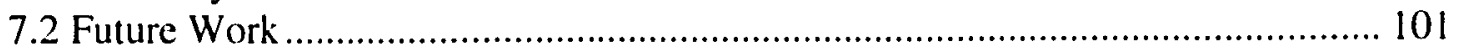

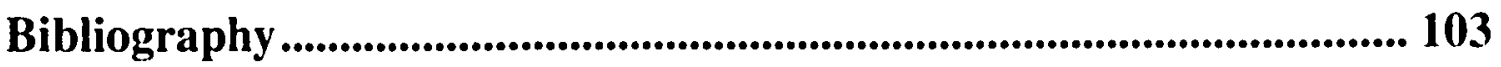




\section{List of Figures}

Figure 2.1 Two-channel subband coding scheme .................................................. 9

Figure 2.2 One dimensional ideal two-channel filter bank structure ....................... 11

Figure 2.3 Two dimensional subband analysis ................................................... 12

Figure 2.4 Two-dimensional subband synthesis................................................... 13

Figure 2.5 Three-levels 2-D DWT decomposition ................................................. 18

Figure 2.6 Two-levels octave-band decomposition of an angiography with an

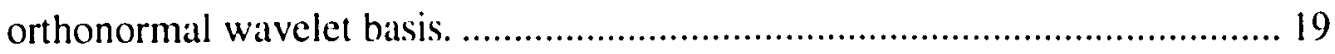

Figure 2.7 Frequency hierarchy of a three-level 2D DWT decomposition .............. 20

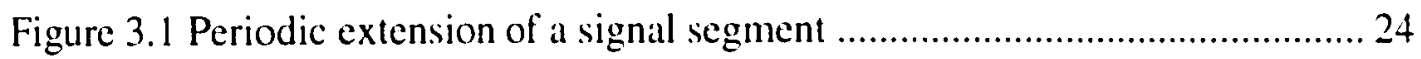

Figure 3.2 Symmetric extension of a signal segment ............................................ 25

Figure 3.3 Analysis of an odd-length signal segment using odd-symmetric

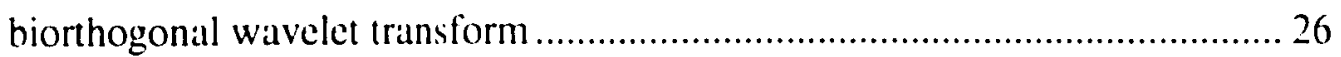

Figure 3.4 Three-levels decomposition of an arbitrary shape ROI of an angiography image

Figure 3.5 Parent-children relationship in octave-band decomposition .................... 31

Figure 3.6 Flowchart of SPIHT algorithm ......................................................... 33

Figure 3.7 Two tier coding scheme employed in JPEG2000................................... 38

Figure 3.8 Scaling and Maxshift methods used in JPEG2000 ................................41

Figure 3.9 Parent-children relationship in SA-DWT subbands................................ 43

vii 
Figure 4.1 3D SPIHT coding scheme ………………............................................ 46

Figure 4.2 Three-levels 3D dyadic decomposition ............................................... 48

Figure 4.3 Parent-children relationship in three dimensions ................................ 50

Figure 4.4 Three-dimensional packet decomposition............................................ 52

Figure 4.5 Symmetric tree structure in packet wavelet decomposition .................... 53

Figure 4.6 Packet tree structure in packet wavelet decomposition........................... 54

Figure 4.7 Optimal 3D coefficient tree structure .................................................. 55

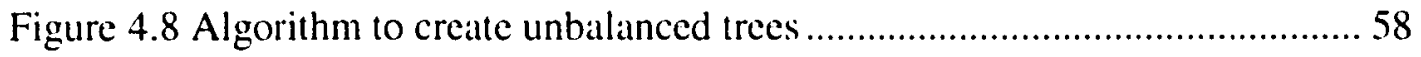

Figure 4.9 Modified 3D SPIHT algorithm based on unbalanced trees...................... 60

Figure 5.1 Subsampling strategy for multiple regions [25] .................................. 64

Figure 5.2 Algorithm to construct MRMQ unbalanced trees ................................. 68

Figure 5.3 Flowchart of 3D MRMQ-SPIHT algorithm ........................................ 70

Figure 5.4 Bitstream structure in 3D MRMQ coding.......................................... 72

Figure 6.I Original slice 10 (left) and slice 20 (right) of image sequence .............. 75

Figure 6.2 Pre-segmented slice number 10 (left) and slice number 20 (right) of

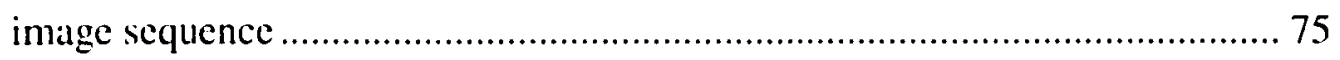

Figure 6.3 Comparison of ROI PSNRs at rate 0.1 bpp......................................... 79

Figure 6.4 Comparison of ROI PSNRs at rate 0.4 bpp.......................................... 80

Figure 6.5 Average ROIs' PSNR of different algorithms at rate from 0.1 to $1.0 \mathrm{bpp}$

viii 
Figure 6.6 Reconstructed slice 32 at rate $0.1 \mathrm{bpp}$ (left, ROI PSNR is $36.03 \mathrm{~dB}$ ) and $0.5 \mathrm{bpp}$ (right, ROI PSNR is $44.06 \mathrm{~dB}$ ) with $\mathrm{GOF}=64$ using UT-SPIHT 82

Figure 6.7 Reconstructed slice 32 at rate $0.1 \mathrm{bpp}$ (left, ROI PSNR is $34.06 \mathrm{~dB}$ ) and $0.5 \mathrm{bpp}$ (right, ROI PSNR is $41.76 \mathrm{~dB}$ ) with $\mathrm{GOF}=16$ using UT-SPIHT 82

Figure 6.8 Zoomed rectangle of reconstructed slice 32 at rate $0.1 \mathrm{bpp}$ (left) and $0.5 \mathrm{bpp}$ (right) with GOF=64 using UT-SPIHT 83

Figure 6.9 Zoomed rectangle of reconstructed slice 32 at rate $0.1 \mathrm{bpp}$ (left) and

0.5 bpp (right) with GOF $=16$ using UT-SPIHT 83

Figure 6.10 Comparison of ROI PSNR 85

Figure 6.11 Reconstructed image slices using MRMQ-SPIHT algorithm by bit allocation among $\mathrm{ROI}$ and background at ratc $0.2 \mathrm{bpp}$ 86

Figure 6.12 Reconstructed image slices by conventional 3D SPIHT at rate 0.2 bpp 86 Figure 6.13 Pre-segmented frame 10 and frame 20 of "Akiyo" 88

Figure 6.14 Results by different 3D compression methods at bit rate 0.5 bpp or $380.16 \mathrm{~kb} / \mathrm{s}$ 89

Figure 6.15 Comparison of performance with different 3D coding methods 90

Figure 6.16 Performance of different GOF sizes for 'Akiyo' using UT-SPIHT algorithm 91

Figure 6.17 Comparison of performance for 'Akiyo' at different temporal transform levels $(\mathrm{GOF}=16)$ 92 
Figure 6.18 Comparison of performance for 'Akiyo' at different temporal transform levels $(\mathrm{GOF}=32)$ 93

Figure 6.19 Comparison of performance for 'Akiyo' at different temporal transform levels $(\mathrm{GOF}=64)$ 9.3

Figure 6.20 Rate-distortion performance for 'Carphone' video using different algorithms 94

Figure 6.21 Comparison of ROI PSNR by MRMQ-SPIHT and conventional dyadic 3D SPIHT on Akiyo video 95

Figure 6.22 Reconstructed "Akiyo" frames of number 10 and 20 at rate $228.096 \mathrm{~kb} / \mathrm{s}$ with $90 \%$ bits for ROI and $10 \%$ bits for background 96

Figure 6.23 Reconstructed "Carphone" frames of number 5 and 15 at rate 228.096 $\mathrm{kb} / \mathrm{s}$ with $90 \%$ bits for ROI and $10 \%$ bits for background 96 


\section{List of Tables}

Table 6.1 Average ROI PSNR (dB) at rate 0.1 bpp with different interslice transform

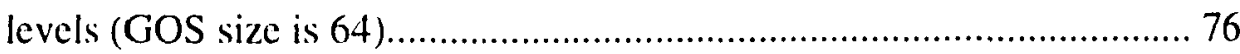

Table 6.2 Average ROI PSNR (dB) at rate 0.4bpp with different interslice transform levels (GOS size is 64)

Table 6.3 Average ROI PSNR (dB) at 0.I lopp for different GOS sizes..................... 77

Table 6.4 Average ROI PSNR (dB) at 0.4bpp for different GOS sizes.................... 77

Table 6.5 Comparison of average ROIs" PSNR $(\mathrm{dB})$ with different 3D coding

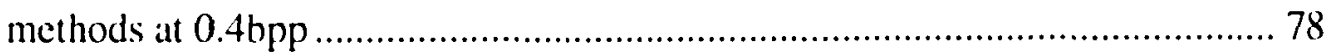




\section{List of Acronyms}

$\begin{array}{ll}\text { 2D } & \text { Two Dimensional } \\ \text { 3D } & \text { Three Dimensional } \\ \text { CB-AC } & \text { Context-based arithmetic coding } \\ \text { CT } & \text { Computerized Tomography } \\ \text { DCT } & \text { Discrete Cosine Transform } \\ \text { EBCOT } & \text { Embedded Block Coding with Optimal Truncation } \\ \text { ESCOT } & \text { Embedded Subband Coding with Optimal Truncation } \\ \text { EZW } & \text { Embedded Zerotree Wavelet } \\ \text { GOF } & \text { Group of Frames } \\ \text { GOS } & \text { Group of Slices } \\ \text { JPEG } & \text { Joint Picture Expert Group } \\ \text { LIP } & \text { List of Insignificant Pixels } \\ \text { LIS } & \text { List of Insignificant sets } \\ \text { LSP } & \text { List of Significant Pixels } \\ \text { MRI } & \text { Magnetic Resonance Imaging } \\ \text { MRMQ } & \text { Multiple-Region Multiple-Quality } \\ \text { MSE } & \text { Mean Squared Error } \\ \text { PACS } & \text { Picture Archiving and Communication System } \\ \text { PCRD } & \text { Post-Compression Rate-Distortion optimization } \\ \text { PET } & \text { Positron Emission Tomography } \\ \text { PSNR } & \text { Peak Signal to Noise Ratio } \\ \text { ROI } & \text { Region of Interest } \\ \text { SA-DWT } & \text { Shape Adaptive Discrete Wavelet Transform } \\ \text { SPECT } & \text { Single Photon Emission Computed Tomography } \\ \text { SPIHT } & \text { Set Partitioning in Hierarchical Tree } \\ \text { US } & \text { Ultrasound }\end{array}$




\section{Chapter 1}

\section{Introduction}

\subsection{Background}

Several of today's imaging technologies generate three-dimensional (3D) datasets. Medical imaging modalities, such as magnetic resonance imaging (MRI), computed tomography (CT), ultrasound (US), single photon emission computed tomography (SPECT) and positron emission tomography (PET) produce multiple slices of images with each slice providing information of a different cross section of the part of the body under examination [2]. Multispectral-imaging techniques generate multiple images of the same scene at different spectral wavelengths. Applications, such as image database and retrieval (e.g. picture archiving and communication systems (PACS), image database browsing), and image transmission (e.g. telemedicine, internet browsing), require an efficient image compression scheme to reduce the cost of image storage and transmission time. For accurate diagnosis, lossless compression of medical image data is required to preserve all the information. However, lossless compression algorithms have a moderate compression ratio, typically in the range of $3: 1$. For higher compression rates, the lossy compression schemes must be employed. Furthermore, for applications such as video 
streaming over internet and video database browsing, there is a growing demand for efficient compression of video data [6]. Conventional video compression using motion compensation and discrete cosine transform (DCT) offers the advantages of high compression ratios and availability of standards for implementation. However, features required by today's applications including low computational complexity and scalability along with good rate-distortion performance are not supported with conventional video coders.

Success of the two-dimensional (2D) embedded image coding algorithms including Set Partitioning in Hierarchical Trees (SPIHT), embedded zerotree wavelet (EZW) and Embedded Block Coding with Optimal Truncation (EBCOT), led the researchers to extend these compression schemes to three dimensions for compression of video and volumetric image data. The main idea is to exploit the interslice/temporal correlation using 3D wavelet transforms and remove the interslice/temporal redundancy. In the case of video data, the motion compensation which is not easily scalable and involves an expensive searching procedure is replaced by wavelet transform applied along the temporal dimension [6]. A three-dimensional (3D) version of EZW has been proposed for compression of volumetric medical images in [2]. 3D dyadic wavelet transform is applied on several CT and MR datasets and then three dimensional EZW coding scheme is adopted to encode resulting subbands. According to the experimental results in [2], 3D EZW algorithm obtained above $20 \%$ decrease, in average, in compressed file size for representation of volumetric medical images compared with 2D SPIHT for lossless 
compression. 3D version of the original SPIHT algorithm using dyadic and packet wavelet transforms have been used for video coding in [9] [10], and also employed for volumetric image compression in [7]. C. He et al. [6] propose a modified 3D coefficient tree structure and obtained better coding performance than 3D SPIHT algorithm based on conventional coefficient tree structure. 3D Embedded Subband Coding with Optimal Truncation (ESCOT) algorithm is directly extended from EBCOT algorithm (used in JPEG2000) for video coding [11], and also applied in volumetric medical data compression [4]. State-of-the-art rescarch in this field focuses on progressive coding of the entire image sequence rather than slice-by-slice coding either in a lossless or lossy fashion according to the required quality constraints.

For certain applications, only specific regions in the volumetric data or video are of interest. For example, in MR imaging of the skull, the physician is mostly concerned about the features inherent in the brain region. In videoconferencing, the speaker's head and shoulders are of main interest and need to be coded at higher quality whereas the background can be either discarded or encoded at a much lower rate. High compression ratios can be achieved by allocating more bit rate for region(s) of interest (ROI) and less bit rate for the remaining regions, i.e. the background. Region-based image coding schemes using heterogeneous (multiple) quality constraints are especially attractive because they not only can well preserve the diagnostic features in region(s) of interest. but also meet the requirements of less storage and shorter transmission time for medical imaging applications and video transmission. 


\subsection{Objective}

The aim of this thesis is to develop 3D region-based image/video coding algorithms. First, we focus on developing a compression scheme for volumetric medical images. Then, the proposed algorithm will be extended for the compression of video data. Previously, regular cuboids are used for specifying the ROIs in volumetric image coding in [8]. Several different types of volume cropping for the specification of a 3D ROI are defined. such as sub-volume, fence, inverted fence, cross and inverted cross. Conventional wavelet transform is applied on CT and MR image datasets and the coefficients belonging to the ROIs are scaled up. Subsequently, conventional SPIHT coding algorithm is used to encode the resulting subbands.

In this thesis, we adopt arbitrary-shape representations for the ROIs, since it is more general and no information from the background region is required in the representation and coding of the ROIs. To represent the arbitrary-shape region, shape-adaptive discrete wavelet transform (SA-DWT) is used to decompose the volumetric images and video data. Note that in conventional embedded image coders, the spatio-temporal orientation trees are heterogeneous when applied to region-based coding, i.e. the coefficients in a particular tree may belong to different ROIs. In this thesis, we propose a region-based coding method that uses unbalanced trees instead of conventional spatio-temporal orientation trees for coding of volumetric images and video data. 


\subsection{Contributions}

The contributions of the thesis are summarized as following:

- A 3D SPIHT coding algorithm based on optimal coefficients tree [6] originally developed for video coding is implemented. We compare its R-D performance for volumetric image coding to conventional 3D SPIHT using both dyadic and packet wavelet decompositions. Experimental results verify that the 3D SPIHT coding algorithm using optimal coefficient tree structure outperforms the conventional 3D SPIHT.

- We propose a 3D version of unbalanced tree structure developed previously for region-based image coding [1]. This method avoids the heterogeneity of the spatiotemporal orientation tree structure such that more coding gains are obtained in the ROIs. We developed a 3D coding scheme using a single wavelet both in spatial and temporal dimensions and then implemented a different version this time using different wavelets along different dimensions. We compared the rate-distortion performance of the two coding schemes for compression of MR image data.

- A main advantage of the proposed technique is that it supports multiple-region multiple-quality (MRMQ) coding using unbalanced orientation trees. By this method. total bit budget can be allocated among multiple ROIs and background accurately, depending on the quality constraints. We implemented a 3D MRMQ coding algorithm and proposed a bitstream structure for multiple ROIs. Experimental results show that this technique offers reasonably well rate-distortion performance for coding multiple ROIs. 
- The proposed algorithm can also be used in video compression in a similar way as the object-based video coding in new MPEG-7 standard. The algorithm uses modified 3D SPIHT coding based on unbalanced tree structure in video compression with arbitrarily shaped ROI(s). This algorithm supports MRMQ coding in video compression, which can allow more bits to be used for encoding the foreground and remaining bits for the background based on the available bit budget.

\subsection{Thesis Outline}

In Chapter 2, we provide an overview of subband and wavelet image transform and coding. The properties of wavelet transform are discussed for the purposes of embedded image compression. Related terminology is also defined at the end of this chapter.

In Chapter 3, we discuss shape-adaptive discrete wavelet transform. We describe the wavelet representation of images by odd-length biorthogonal wavelet basis. Then. the state-of-the-art bit plane coders. SPIHT and JPEG2000, and the ROI coding methods used by conventional SPHIT and JPEG2000 are discussed.

The extension of conventional 2D SPIHT coding to three dimensional data is presented in Chapter 4. Two 3D wavelet decomposition schemes, dyadic and packet transforms, are introduced next. Then, we discuss the implementation of a 3D optimal coefficient tree algorithm. Finally, we describe a 3D ROI image coding technique based on unbalanced tree structures. 
In Chapter 5, we describe the wavelet representation of multiple ROIs and present the 3D MRMQ coding algorithm.

Experimental results are given in Chapter 6. R-D performance of volumetric medical datasets and video sequences using the proposed ROI coding technique is compared with that of state-of-the-art 3D coding methods based on original SPIHT algorithm.

Finally, summary and future work are given in Chapter 7. 


\section{Chapter 2}

\section{Subband Transform Coding and Wavelet}

\section{Transform}

In this chapter we give an overview of subband and wavelet image transform. Subband transform is a simple yet powerful technique for data compression to achieve energy compaction. The wavelet transform can be viewed as a special case of subband transform based on approximation theory instead of frequency analysis, which has shown to be more suitable for image compression [11] [15] [16]. Although arbitrary-shape wavelet representations exhibit similarities to conventional wavelet transform, some important differences should be examined for improving the performance of the subsequent regionbased coding.

The chapter is organized as follows: In section 2.1, subband transform coding is described. A brief theory of wavelets and wavelet transform are discussed in section 2.2 and 2.3, respectively. Finally, related terminology is given in the last section. 


\subsection{Subband Coding}

Subband transform coding technique partitions the source signal into different frequency subbands using digital filters applied in a cascade of stages so that the source signal can be represented only by a few frequency components [16]. The output of the filters are subsequently encoded and transmitted over the communication channel. Such energy compaction makes it more efficient to code the frequency components of a signal than the time domain signal itself. The basic subband coding scheme implemented using a twochannel filter bank is shown in Figure 2.1 as an example.

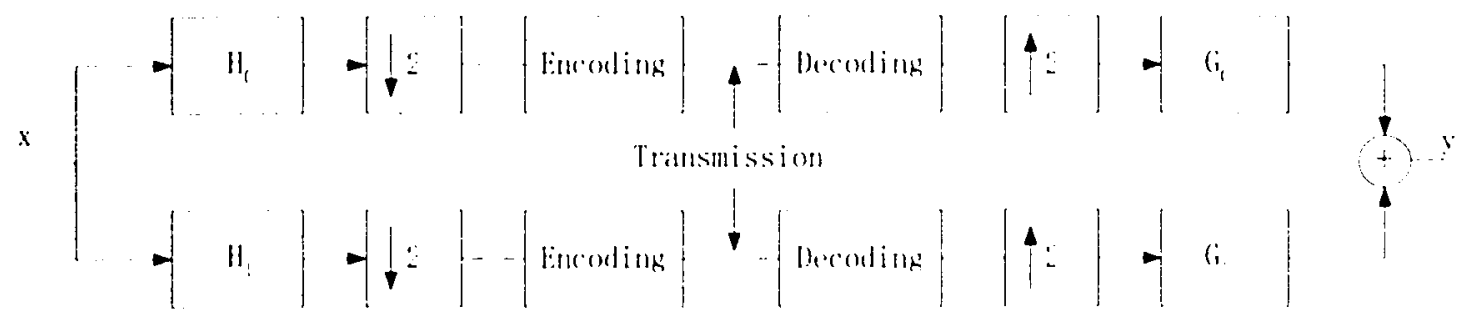

Figure 2.1 Two-channel subband coding scheme

In this scheme, the input signal is passed through a bank of two filters, called the analysis filter bank which covers the range of frequencies that make up the input signal. The output of the filters is downsampled by two to produce two new representations of input signals based on filter functions. Filters $H_{0}$ and $H_{1}$ are ideal lowpass and highpass filters. The downsampling operation is justified according to the Nyquist rule and its generalization. That is, we only need to keep twice as many samples per second as the range of frequencies to restore a signal without aliasing. For two-channel subband 
decomposition, the frequency range of the input is twice as long as that of the output of the filters, so we can reduce the number of samples by a factor of two. After downsampling, a coding scheme is used to encode the output. In the synthesis stage, the coded samples from each subband are decoded and then upsampled by inserting zeros between consecutive samples to bring the number of samples back to the original rate. Next, these upsampled signals are filtered by synthesis lowpass and highpass filters $G_{0}$, and $G_{1}$ respectively and then added together to reconstruct the input data.

\subsubsection{Analysis Stage}

For decomposing a source signal into its subband components, we can choose multiple filter banks with different characteristics. However, in image coding a more popular method is to apply two-channel filter bank structure repeatedly to form a cascaded structure [13] [16] [17]. A two-level hierarchical structure is as shown in Figure 2.2. The output from the low-pass filter is then input to the next stage of the filter bank. We can thus obtain multiple representations of a source signal. The advantage of this structure is that it is implemented by simply repeating the two-channel subband analysis, resulting in efficient signal decomposition. 


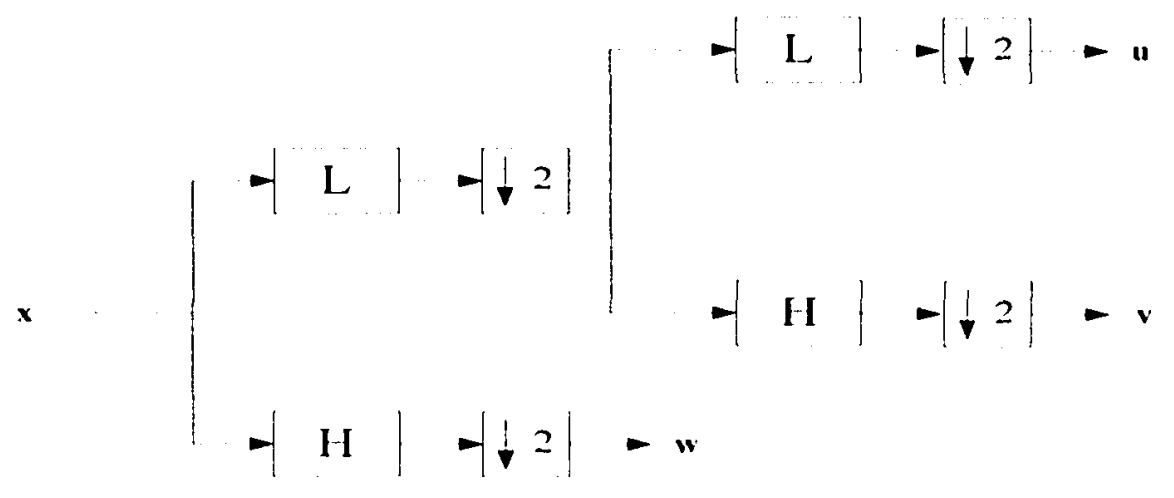

Figure 2.2 One dimensional ideal two-channel filter bank structure

In image compression, such a decomposition scheme should be extended to twodimensions. By choosing separable filters. a two-dimensional subband coding scheme can be implemented as a straightforward extension of the one-dimensional version. This is performed by applying the same filters along the two dimensions iteratively. For an $\mathrm{M}$ x $M$ image, $M$ one-dimensional transforms are carried out along the rows (one for each row). This results in two $\mathrm{M} \times \mathrm{M} / 2$ subimages/subbands, one corresponding to the lowpass filtered components and the other corresponding to the high-pass filtered rows of the image. Next, each of these subbands is filtered further along the columns to partition the resulting data into four $M / 2 \times M / 2$ subbands (low-pass row and low-pass column, lowpass row and high-pass column, high-pass row and low-pass column, high-pass row and high-pass column). This constitutes one stage of the subband decomposition of an image, and is depicted in Figure 2.3. In this thesis, we are concerned with 3D datasets, so one stage of decomposition results in eight sub-volumes. This is accomplished by transforming each slice of the volumetric dataset using two-dimensional transform and 
then applying one-dimensional transform along the slices. The reader is referred to [16][22] for a more detailed discussion of subband transform and coding.

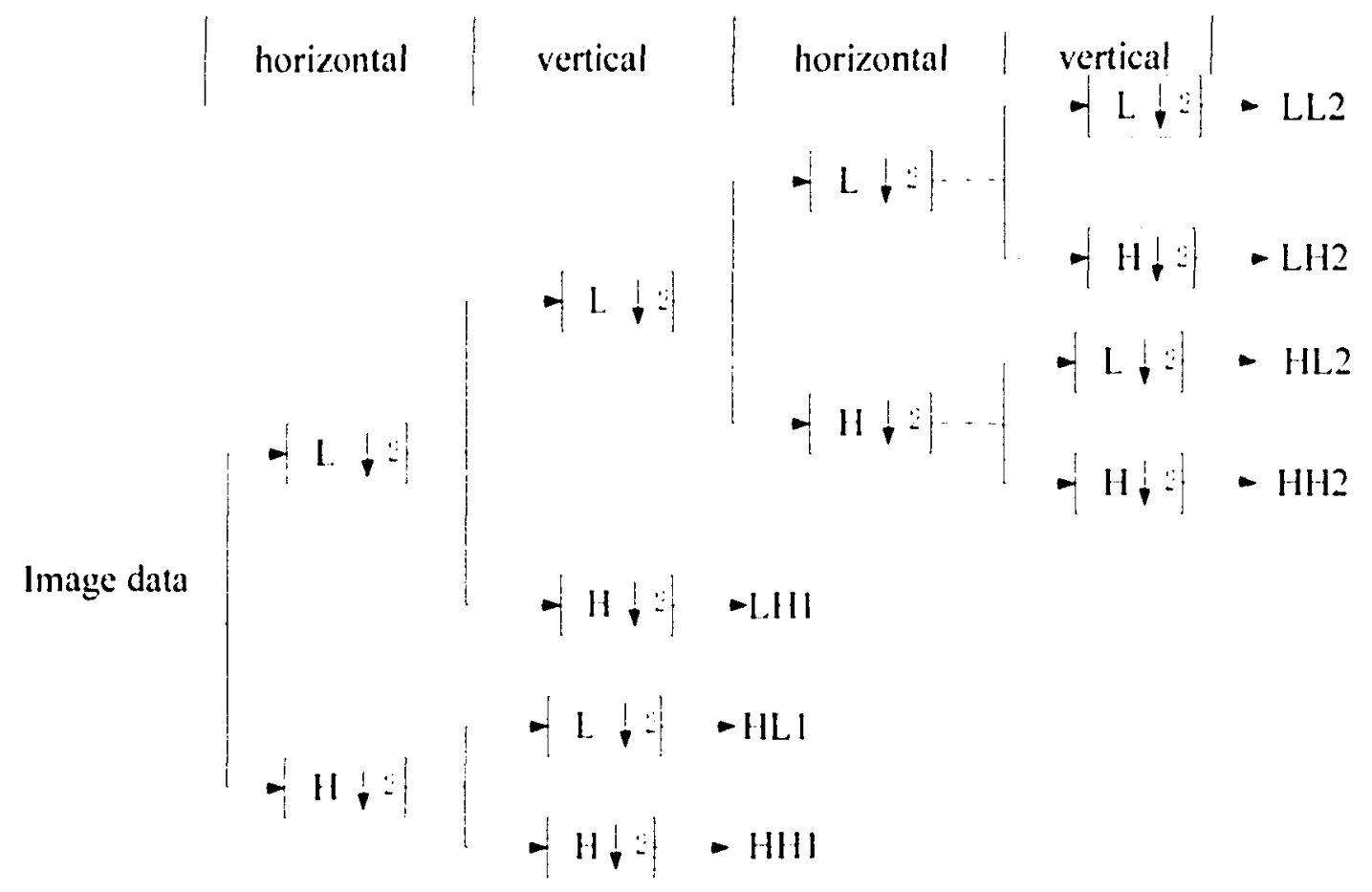

Figure 2.3 Two dimensional subband analysis

\subsubsection{Coding Stage}

Once the image is decomposed into subbands, an encoding scheme is used to compress the transform coefficients. Traditionally, scalar quantization, vector quantization and differential encoding algorithms are used in this stage [16]. Two state-of-the-art coding schemes based on wavelet transform, SPIHT and JPEG2000, employ zerotree coding which will be discussed in the following chapter. 


\subsubsection{Synthesis Stage}

In the previous section, we described the subband decomposition of images into different frequent components. In order to reconstruct the input image, a synthesis scheme is needed to restore the original input. The process of decomposition can be reversed, so it is possible to obtain reconstructed image given that the decomposed components are available. Such synthesis process can be realized by up-sampling followed by filtering, and then combining the results at the output of the filters. For the analysis scheme given in Figure 2.1, corresponding synthesis scheme is shown in Figure 2.4.

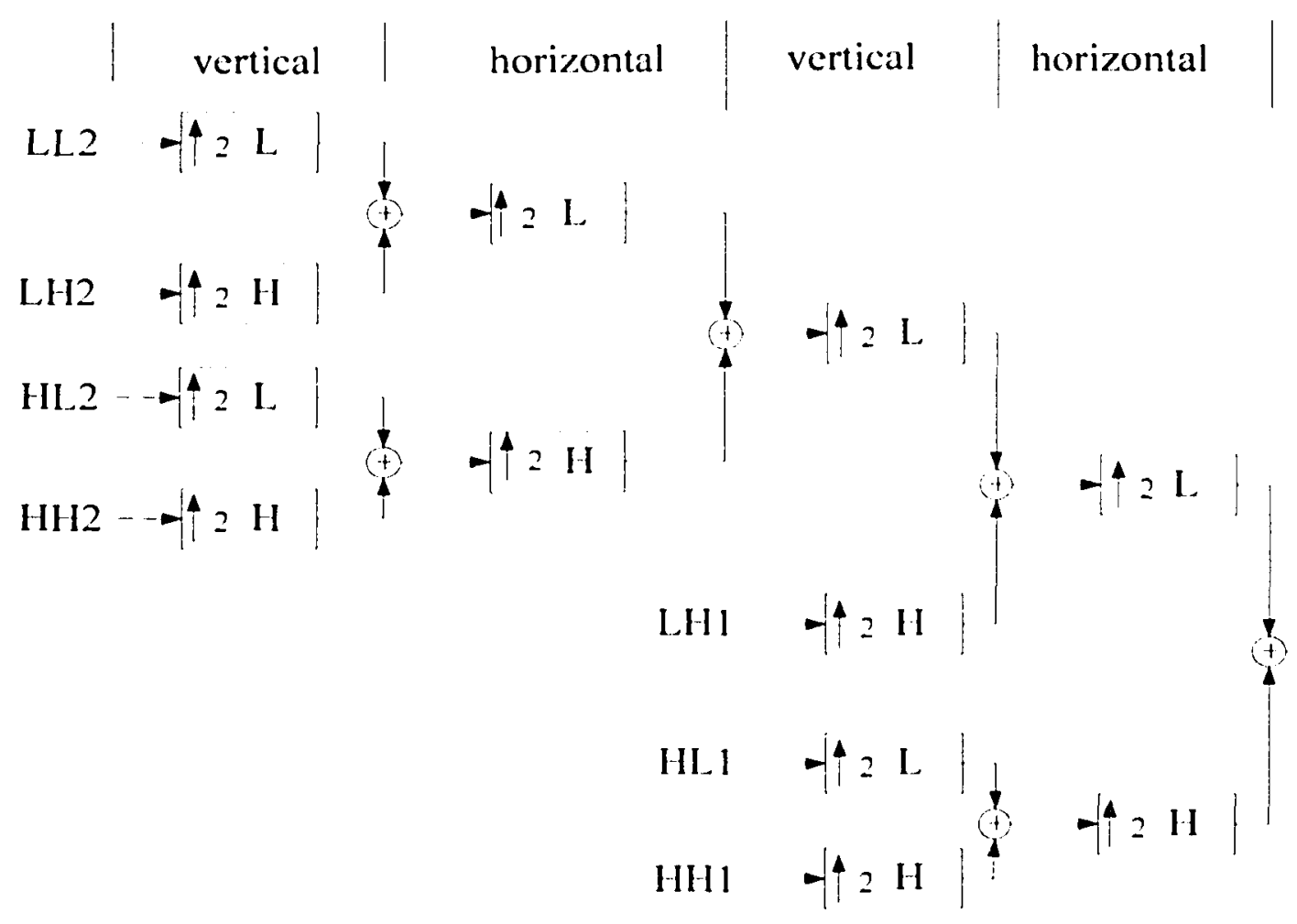

Figure 2.4 Two-dimensional subband synthesis 


\subsection{Wavelets}

The wavelet transform can be viewed as a special case of sub-band decomposition and has been extensively studied in image and video coding. Wavelets provide an efficient means for approximating source signal with a small number of basis elements [11]. In general, the wavelets are a set of functions that are generated from a single function, $\psi(t)$, called the mother wavelet. The family of wavelets can be defined as:

$$
\psi_{u t}(t)=|a|^{-1 / 2} \psi\left(\frac{t-\tau}{a}\right)
$$

Where $a$ and $\tau$ are time dilation and translation parameters, respectively.

For discrete application, the dilation parameter a takes the values $2^{k}$ and the translation parameter $\tau$ is chosen as $2^{k} m$, where $k$ and $m$ are both integers. In this case, equation 2.1 becomes:

$$
\psi_{k, m}(t)=2^{-\frac{1}{t}} \psi\left(2^{-k} t-m\right)
$$

These wavelets are used to decompose the image data into a superposition of dilated I shifted wavelets. So $x(t)$ can be written as:

$$
x(t)=\sum_{k, m} \alpha_{k, m} \psi_{k, m}(t)
$$


Here $\alpha_{k, m}$ are called the wavelet transform coefficients of $x(t)$ and can be obtained with equation 2.4 .

$$
\alpha_{k, m}=2^{-\frac{1}{2}} \int x(t) \psi\left(2^{-k} t-m\right) d t
$$

The wavelet transform is suitable for the analysis of natural images because it provides a good representation of the image data in terms of both spattial and frequency localization. Natural images are locally smooth, and their frequency characteristics vary in space. The Fourier transform and DCT are able to capture the global frequency characteristics of a signal, but they do not take into account the fact that different regions in an image may have different frequency characteristics. The scaling property of the wavelet transform provides information about frequency characteristics and where these characteristics are localized in space. The coefficient $\alpha_{h, m}$ characterizes the projection of $x(t)$ onto the basis function, $\psi_{k, m}(t)$. For different $k, \psi_{k, m}$ represents different frequency characteristics. $m$ is the translation parameter of the dilated mother wavelet. Therefore, $\alpha_{k, m}$ can be considered as the combined space-frequency characteristics of the signal. Details of wavelet transform are given in $[16]-[21]$.

\subsection{Wavelet Transform}

For image coding, the wavelet transform is used to decompose the image data into different frequency subbands. Therefore, the wavelet transform is equivalent to 
computing the output of a band-pass filter bank specified by the wavelets of $\psi_{k, . m}(t)$ [16] [17] [19]. Thus, the main application of wavelet theory is in the design of filters for subband coding. Some considerations about wavelets are briefly discussed next.

\subsubsection{Properties of Wavelets}

As the number of vanishing moments of a wavelet increases, the wavelet becomes smoother. Wavelet transform using a smooth wavelet provides a good approximation of smooth signals. For images with mostly smooth regions, a large number of vanishing moments is desirable for the wavelet to achieve high energy compaction. Where the image is smooth, most of the wavelet coefficients are zero, therefore a small number of coefficients can be used to represent the image data. This is the main objective of subband image coding.

Orthonormality is another desired property of the wavelet transform. A wavelet transform based on an orthonormal wavelet preserves the conservation of energy in the transform domain. This is especially important if a lossy coding scheme is applied to the transformed data. The orthonormal wavelet is implemented as a unitary transform. This guarantees that the distortion in the transform domain is the same as in the reconstructed image domain $[17,19]$ (i.e. the distortion introduced by quantizing transform coefficients will be the same as that of the quantized components after inverse transformation.) 
Therefore, control of distortion in the reconstructed image domain can be achieved by controlling the distortion in the transform dontiin.

Besides smoothness and orthonormality, short filter length and linear phase properties are desirable in choosing a wavelet basis for image compression. Short filters make transform computation fast. Also, long filters tend to spread encoding errors. Without the linear phase property, the phase distortion around edges would be visible. In addition, using a linear phase wavelet make it possible to cascade filters in a subband coding scheme without phase compensation. A symmetric wavelet, which has a property of linear phase, can remove artificial oscillations due to image edges. Thus, the use of symmetric wavelet is often needed in image coding.

Since linear phase and orthonormality are mutually exclusive, biorthogonal wavelets are usually employed instead. The linearity and smoothness properties can still be achieved. However with biorthogonal bases used in the filter design, the transform is no longer orthonormal. Therefore, distortion control in the reconstructed image domain can not be achieved by handling the distortion in the transform domain. Nevertheless, we can choose the biorthogonal basis close to orthonormal so that distortion in the transform domain approximately corresponds to the distortion in the reconstructed data [16] [17]. 


\subsubsection{Representation of Spatial Hierarchies}

There exist both spatial and frequency hierarchies between different subbands in the discrete wavelet transform. The spatial hierarchy for an image can be visualized as shown in Figure 2.5.

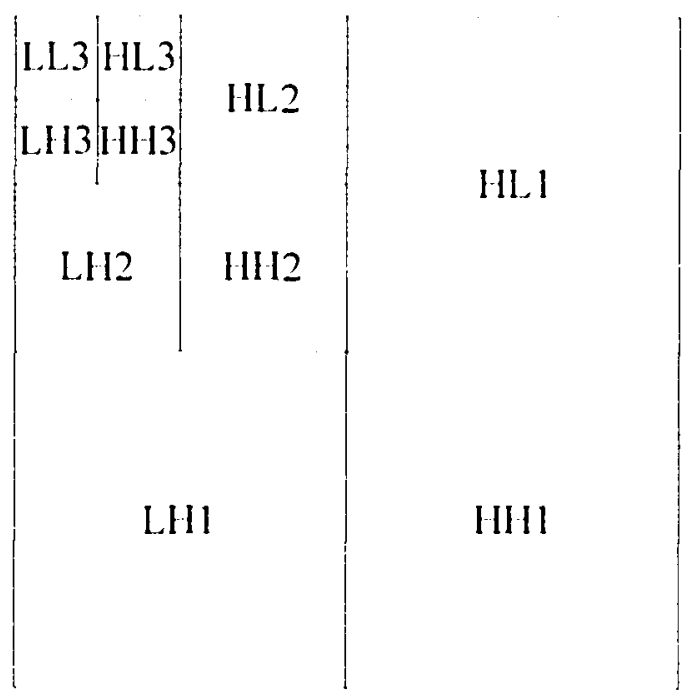

Figure 2.5 Three-levels 2-D DWT decomposition

In Figure 2.5 the letters correspond to the subbands where filters applied in horizontal and vertical direction respectively. For example, subband LHI is obtained by applying lowpass filter on image data in horizontal direction first and then filtering output by highpass filter in vertical direction. To illustrale the spatial correlation, two-level octaveband decomposition for a $512 \times 512$ grey scale image (Angio) is shown in Figure 2.6. The edges in the image running in vertical direction are evident in the HLI subband of the transformed image. The four subbands in the upper left corner of the DWT 
decomposition in Figure 2.6 are all produced by applying the lowpass and highpass filters to the approximation subband resulting from the first decomposition stage. It can be seen that the resulting subband HL2 also contains this edge. The subband data have been rescaled so that the largest coefficient in each subband maps to 255 and the smallest to 0 . Hence, there is a correlation between the HL1 and HL2 subbands. The same also applies for the $\mathrm{LH} 2, \mathrm{LH} 1$ and $\mathrm{HH} 2, \mathrm{HH} 1$ subbands, which are subbands at different levels but containing features with the same orientation. This correlation should be exploited by the coding scheme employing the DWT.

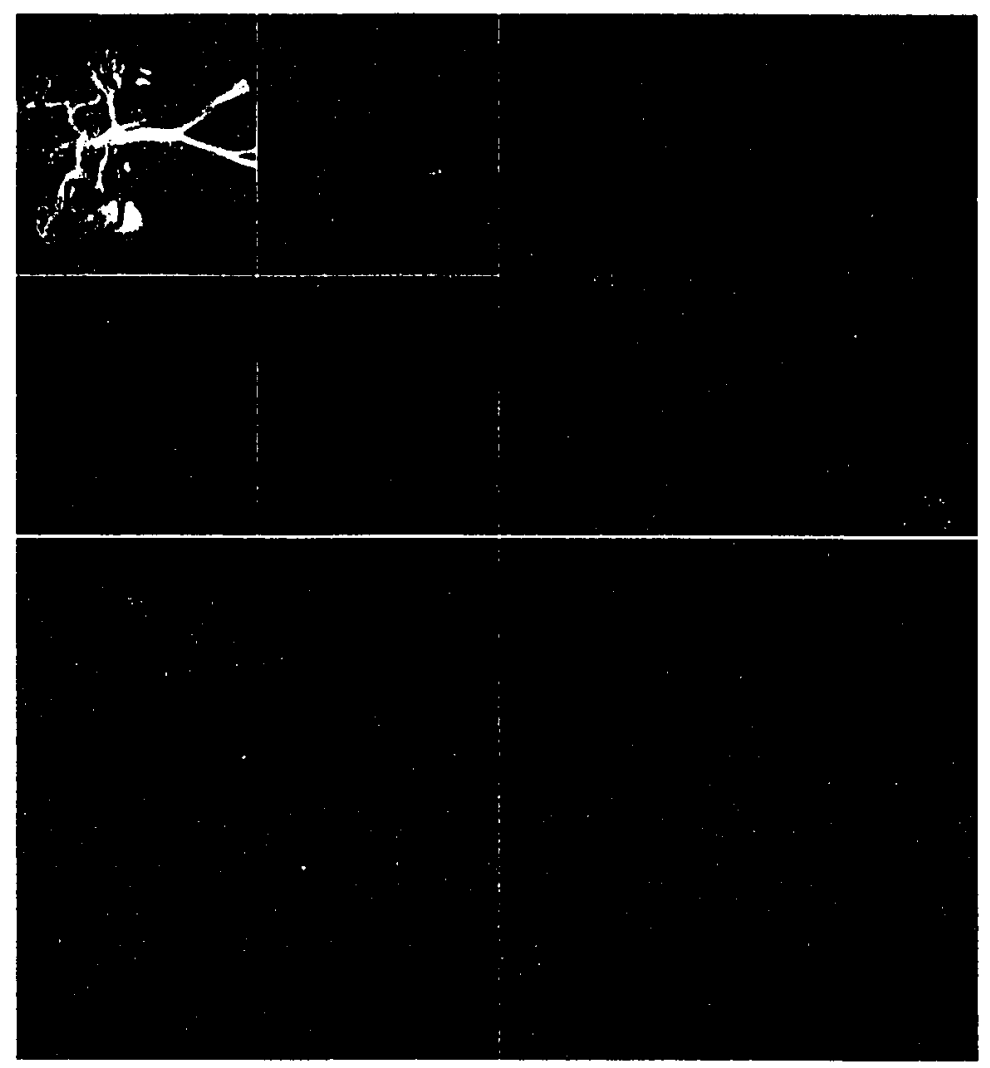

Figure 2.6 Two-levels octave-band decomposition of an angiography with an orthonormal wavelet basis. 


\subsubsection{Representation of Frequency Hierarchies}

In addition to the spatial hierarchy, there is also frequency hierarchy among subbands. A tree structure is used to illustrate this hierarchical relationship between the subbands. The frequency hierarchy among the subbands from Figure 2.5 is depicted in Figure 2.7. For example, LL3 subband is parent of LH3 subband and LH3 subband is parent of LH2 subband and so on. They are different frequency subbands with the same characteristics.

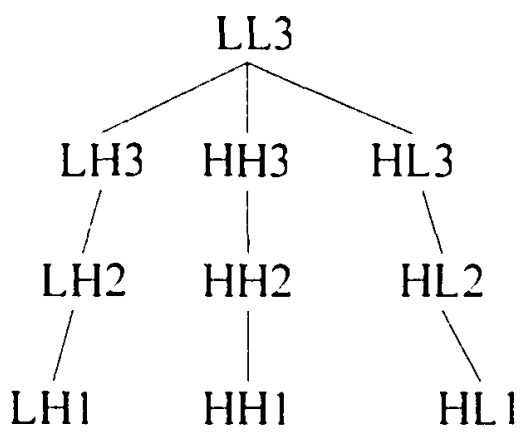

Figure 2.7 Frequency hierarchy of a three-level 2D DWT decomposition

\subsubsection{Extension to Third Dimension}

Both the spatial and frequency relationships between subbands have been illustrated in two dimensions; extension to third dimension is straightforward. In three dimensions the spatial hierarchy becomes a hierarchy of sub-volumes. At each stage the desired subbands are split up into eight sub-volumes structured into a cube. The frequency hierarchy trees will be similar to those shown in Figure 2.7, but with a branching factor of eight. 


\subsection{Terminology in the Thesis}

The definitions of the terminology used in this thesis are given below. Images and image volumes/video sequences will be viewed as special cases of two- and three-dimensional signals where pixel values represent signal samples.

The signal energy is defined as:

$$
E=\sum x_{1}^{2}
$$

and orthogonality between signals will be defined as

$$
x \perp y \Leftrightarrow \sum x_{1} y=0
$$

When lossy compression is employed, we need to measure the difference (distortion) between the original signal and the reconstructed one. A common metric for measuring distortion is the Mean Squared Error, or MSE. In the MSE measurement the total squared difference between the original signal and the reconstructed one is averaged over the entire signal and can be computed as follows:

$$
M S E=\frac{1}{N} \sum_{i=11}^{N-1}\left(\hat{x}_{i}-x_{i}\right)^{2}
$$


where $N$ is the total number of samples of signal, and $\hat{x}_{i}$ is the reconstructed value of $x_{i}$. Another distortion measure is the Peak Signal to Noise Ratio, or PSNR. Since the MSE is sensitive to the range of signal scale, the PSNR is used to incorporate the maximum amplitude of the original signals into the metric, making this measurement independent of the range of the data. PSNR is usually measured in decibels as:

$$
P S N R=10 \log _{10}\left(\frac{x_{\text {Prat }}^{2}}{M S E}\right)[d B]
$$

For 8 bit images or image volumes for instance, $x_{\text {prat }}=255$.

\subsection{Summary}

In this chapter, we gave an overview of the subband coding and wavelet transform. Some properties of wavelets are discussed from the viewpoint of image compression. We also discussed the inherent correlation between the subbands of a wavelet transformed image. Next, we will discuss the shape-adaptive discrete wavelet transform and region-based coding. 


\section{Chapter 3}

\section{Shape-Adaptive DWT and ROI Coding}

In the previous chapter, wavelet transform and its use in image compression were presented. To support region of interest (ROI) coding, it is necessary to identify the wavelet transform coefficients associated with the ROI. Commonly, the number of transform coefficients that are related to the reconstruction of ROI is different to that of the ROI in image domain. We have to keep track of the coefficients that are involved in the reconstruction of ROI through each stage of decomposition. Shape adaptive discrete wavelet transform (SA-DWT) provides a simple solution to this problem. SA-DWT has two significant features. One is that the number of coefficients after SA-DWT is identical to the number of pixels in the ROI of the original image: the other is that spatial correlation and locality properties of wavelet transform and self-similarity across subbands are well preserved after SA-DWT [3]. These features make bit-plane coding. such as the one used in SPIHT, still applicable for encoding ROIs in an image. It is shown that SA-DWT is capable of achieving high coding efficiency for arbitrary shape region-based coding [3]. 
In the following section, SA-DWT is described. State-of-the-art image coders, SPIHT and JPEG2000, are presented in section 3.2. Finally, in section 3.3 we discuss how these coders can be extended to support region-based coding.

\subsection{SA-DWT}

\subsubsection{Extension of Finite Length Signals}

An image is of a finite length signal. When wavelet transform is applied to an image, the pixels outside the image borders need to be defined in relation to the pixels along the image borders. This operation is called boundary extension. Two common boundary extension methods used are: periodic extension and symmetric extension.

Periodic extension of a signal segment is illustrated in Figure 3.1. The signal segment is repeated at the boundaries (leading and tailing). The drawback of this method is that there can be discontinuities and less correlation between pixels along the extension boundaries if the signal segment is long. Such transition at the segment boundary will bring out large high frequency coefficients that can considerably degrade the compression efficiency [3] [13].

$$
\text { d } \quad c: c|c| a|b| c|\ldots| d|c| c|a| c: c:
$$

Figure 3.1 Periodic extension of a signal segment 
Symmetric extension can be achieved in different ways [3]. Figure 3.2 illustrates three kinds of symmetric extension of a signal segment. In this thesis, type B symmetric extension is adopted. In this scheme, the signal segment is extended symmetrically at its leading and tailing boundaries. The neighboring pixels at signal extension boundaries have the same close correlation as the neighboring pixels close to the boundaries in the original segment so that smooth transitions can be achieved along the segment boundaries.

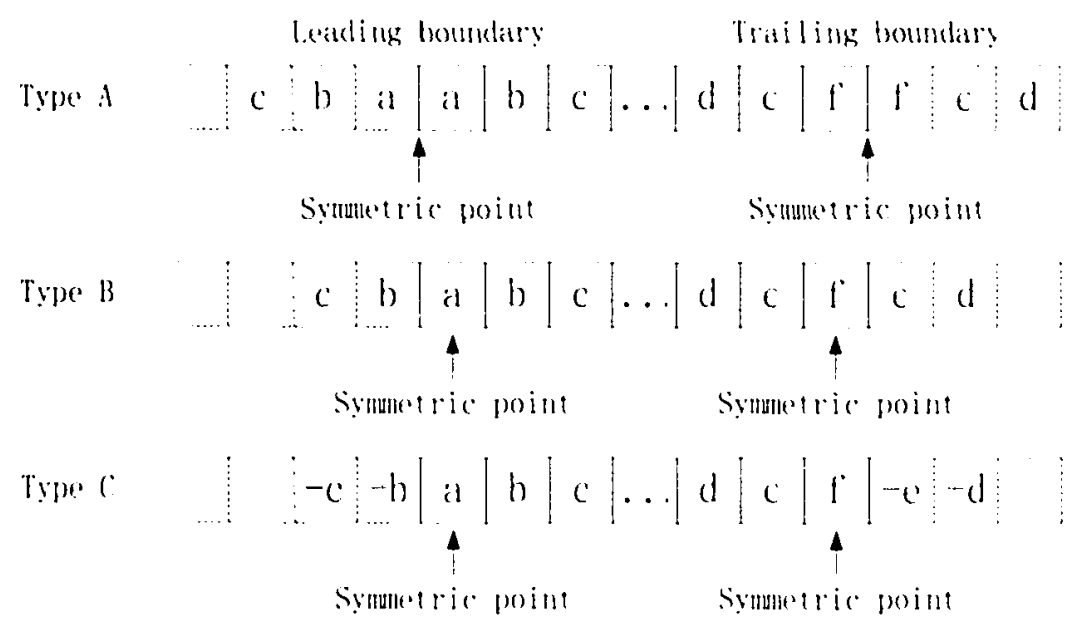

Figure 3.2 Symmetric extension of a signal segment

\subsubsection{Wavelet Representation with Odd-Length Biorthogonal Wavelet Basis}

As discussed in the previous chapter, biorthogonal wavelets will be used in our ROIbased volumetric image compression because they are of linear phase and phase distortion by magnitude distortion of transformed coefficients can be removed [3]. Figure 
3.3 shows finite odd-length signal analysis by symmetric extension and using odd-length biorthogonal wavelet.

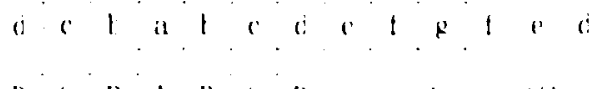

D) $(B \cap B(D)$

1) $(B \quad B \quad B \quad D$

D) $(B \quad A B(B)$

1) $(B A B(B)$

Figure 3.3 Analysis of an odd-length signal segment using odd-symmetric biorthogonal wavelet transform

To calculate the wavelet representation of finite length signal $x[n]$ with length $\mathrm{N}$ $(x[n]=[\operatorname{abcdefg} \mid$ in Figure 3.3), $x[n]$ is extended to infinite length signal $x[n]$ using type B symmetric extension. $\tilde{x}[n]$ can be expressed by equation 3.1 .

$$
\tilde{x}[n+l(2 N-2)]=\left\{\begin{array}{lr}
x[n], & \text { if } l=0 \\
x[2 N-2+n], & \text { if } l \in Z, \neq 0
\end{array}\right.
$$

The prototypes of odd-symmetric biorthogonal lowpass and highpass analysis filters are given as [DCBABCD] and [TSRST] respectively. Filtering extended signal $x[n]$ with these filters and subsampling the result, we obtain representation of the signal $x[n]$ in wavelet domain. Due to the symmetry of extended signal $\tilde{x}[n]$ and analysis filters, the wavelet domain representation $\tilde{y}[n]$ also has the same symmetric form as $\tilde{x}[n]$, and can 
be expressed in terms of $y[n]=\{k, t, l, u, m, v, n\}$. In order to achieve more signal processing gain, global even-subsampling for lowpass coefficients and odd-subsampling for highpass coefficients are applied instead of choosing locally fixed even-subsampling for lowpass coefficients and locally fixed odd-subsampling for highpass coefficients [3].

An important feature of SA-DWT is that the spatial correlation, locality and shape of the ROI are all well preserved. An example of 2D SA-DWT of an arbitrary-shape region is illustrated in Figure 3.4. SA-DWT retains the multi-resolution property of wavelet transform, which is decomposition of an image into different resolution levels [13] [15] [16]. A low resolution reconstruction of an arbitrary shape ROI can be obtained using lowest frequency subband coefficients; incorporating higher frequency subband coefficients progressively, higher resolution reconstruction can be obtained. 


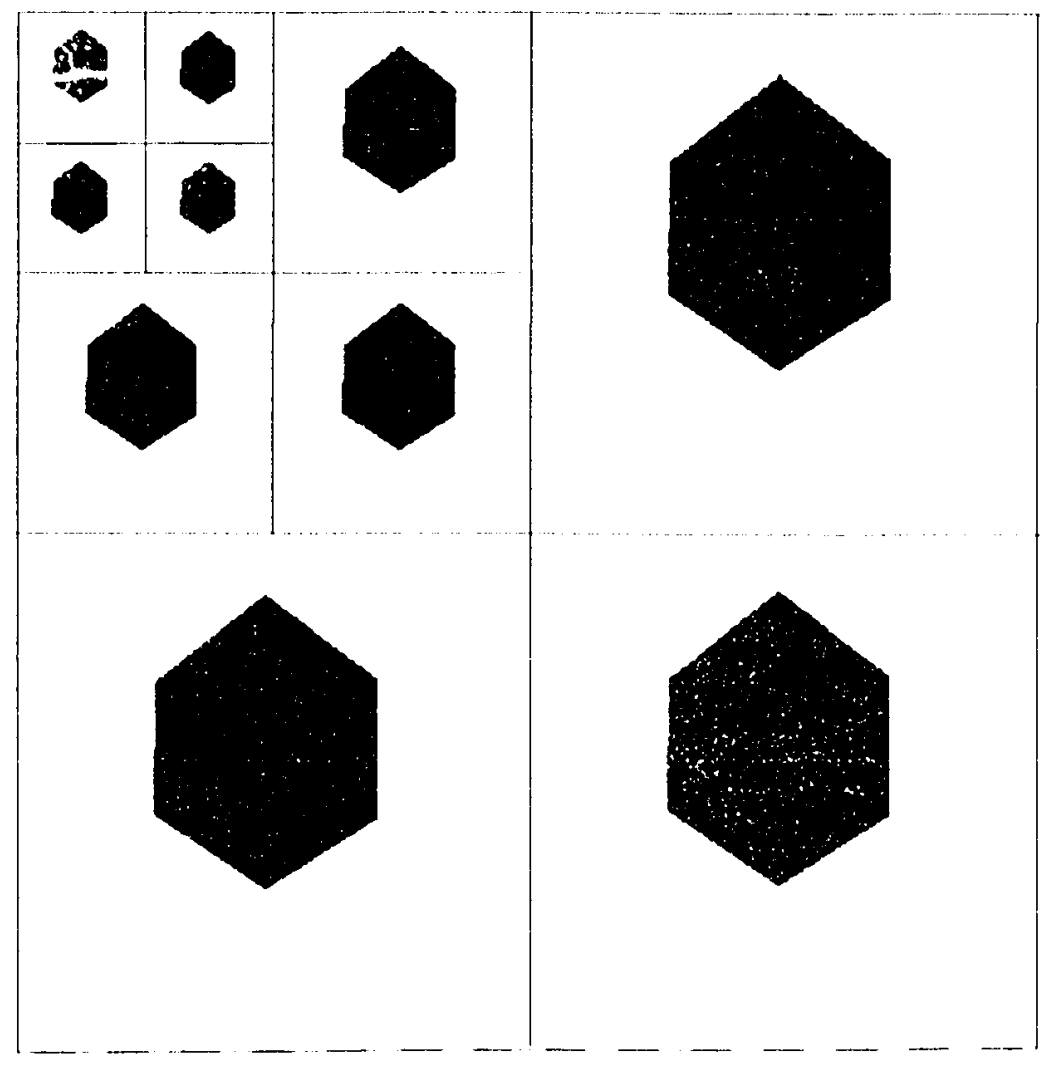

Figure 3.4 Three-levels decomposition of an arbitrary shape ROI of an angiography image

\subsection{Wavelet Bit Plane Coding}

As discussed in previous chapter, wavelet theory provides a way to design filters used in subband coding which splits the spectrum of an image into several frequency subbands. These subbands then can be encoded separately. Energy compaction and multi-resolution property are achieved by hierarchical subband decomposition scheme. For image compression applications, quantization and coding of frequency coefficients are needed to achieve compression. Quantization of coefficients results in information loss and thus 
this step is included only in a lossy compression scheme. At the receiver end, the compressed bit stream will be decoded and inverse wavelet transform will be applied to reconstruct the source image.

State-of-the-art coding schemes, SPIHT [24] and JPEG2000 [13] [14], both use wavelet transform prior to coding and produce embedded bit streams that provide the features of resolution and distortion scalability. Thus, we can reconstruct the source image at low spatial resolution and low quality from part of the embedded bit stream. By progressively decoding more bits, higher spatial resolution and better quality image will be obtained. SPIHT exploits inter-scale dependencies between wavelet coefficients based on set partitioning sorting and spatial orientation tree. JPEG2000 is the still image compression standard developed by Joint Photographic Experts Group (JPEG). JPEG2000 takes advantage of intra-scale dependencies to achieve high compression ratios.

\subsubsection{SPIHT Coding Algorithm}

Set Partitioning in Hierarchical Trees (SPIHT) algorithm is based on embedded zerotrec wavelet (EZW) coding method; it employs spatial orientation trees and uses set partitioning sorting algorithm [24].

An octave-band decomposition structure is used in SPIHT. Cocfficients corresponding to the same spatial location in different subbands in the pyramid structure 
display self-similarity characteristics. Note that, SPIHT defines parent-children relationships between these self-similar subbands to establish spatial orientation trees. Such parent-children relationships in two-dimensional case can be expressed as in equation 3.1. An example showing octave-band decomposition is depicted in Figure 3.5.

$$
\begin{aligned}
& \text { parent }=(x, y) \\
& \text { children }=[(2 x, 2 y),(2 x+1,2 y),(2 x, 2 y+1),(2 x+1,2 y+1)]
\end{aligned}
$$

The arrows in the figure indicate the parent-children relationship in subband pyramid. The start of arrow line is parent coefficient, and end of arrow indicates four children coefficients. The coefficients in the shaded area are all the descendants of root coefficient, A. It is assumed that lower frequency subbands have more energy and if the magnitude of a parent coefficient in a lower frequency subband is less than a threshold, it is highly likely that magnitudes of its descendants are also less than that threshold. Thus, significance map of transformed coefficients with a preset threshold can be efficiently coded by a zero-tree coding scheme. For most images, the assumption used for the construction of spatial orientation tree is satisfied. 


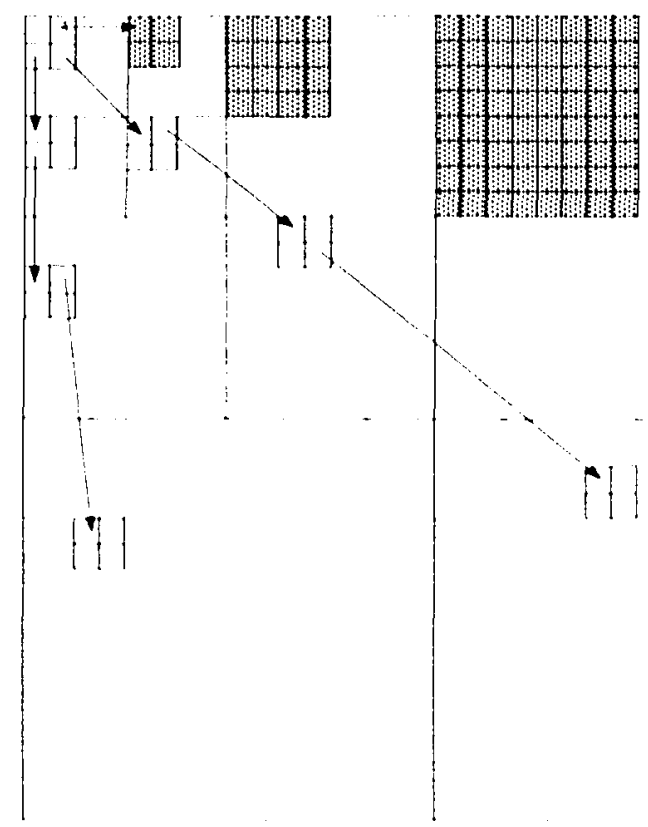

Figure 3.5 Parent-children relationship in octave-band decomposition

The next step is the set partitioning sorting algorithm, which consists of two passes: sorting pass and refinement pass. In sorting pass, the ordering information of the coefficients is found according to the results of a magnitude test. In the refinement pass. the quantization for each significant coefficient is refined in a successive manner. A quantization threshold is used in coefficient magnitude test. The threshold is initialized as

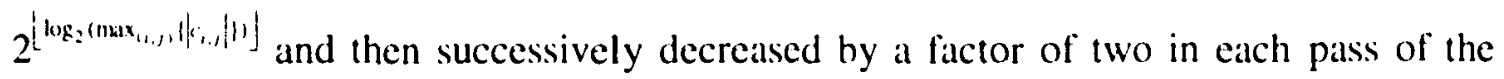
algorithm. When the bit budget is reached, the algorithm will stop.

Three ordered lists, in which each element indicates coordinate pair $(i, j)$ of a coefficient, are used to implement the coding algorithm. They are named as List of Insignificant Pixels (LIP), List of Significant Pixels (LSP) and List of Insignificant Sets 
(LIS). Magnitude test is performed to decide whether a coefficient is significant or not. A coefficient is said to be significant at a given threshold if its magnitude is larger than or equal to that threshold. The construction of LIP, LIS and LSP takes place according to the significance of the coefficients with respect to the threshold value.

- LIP contains coordinates of coefficients that are insignificant with respect to the current threshold

- LSP contains the coordinates of coefficients that are significant at the same threshold

- LIS contains coordinates of the roots of the spatial orientation parent-children trees

The flowchart given in Figure 3.6 illustrates how the SPIHT algorithm works. 


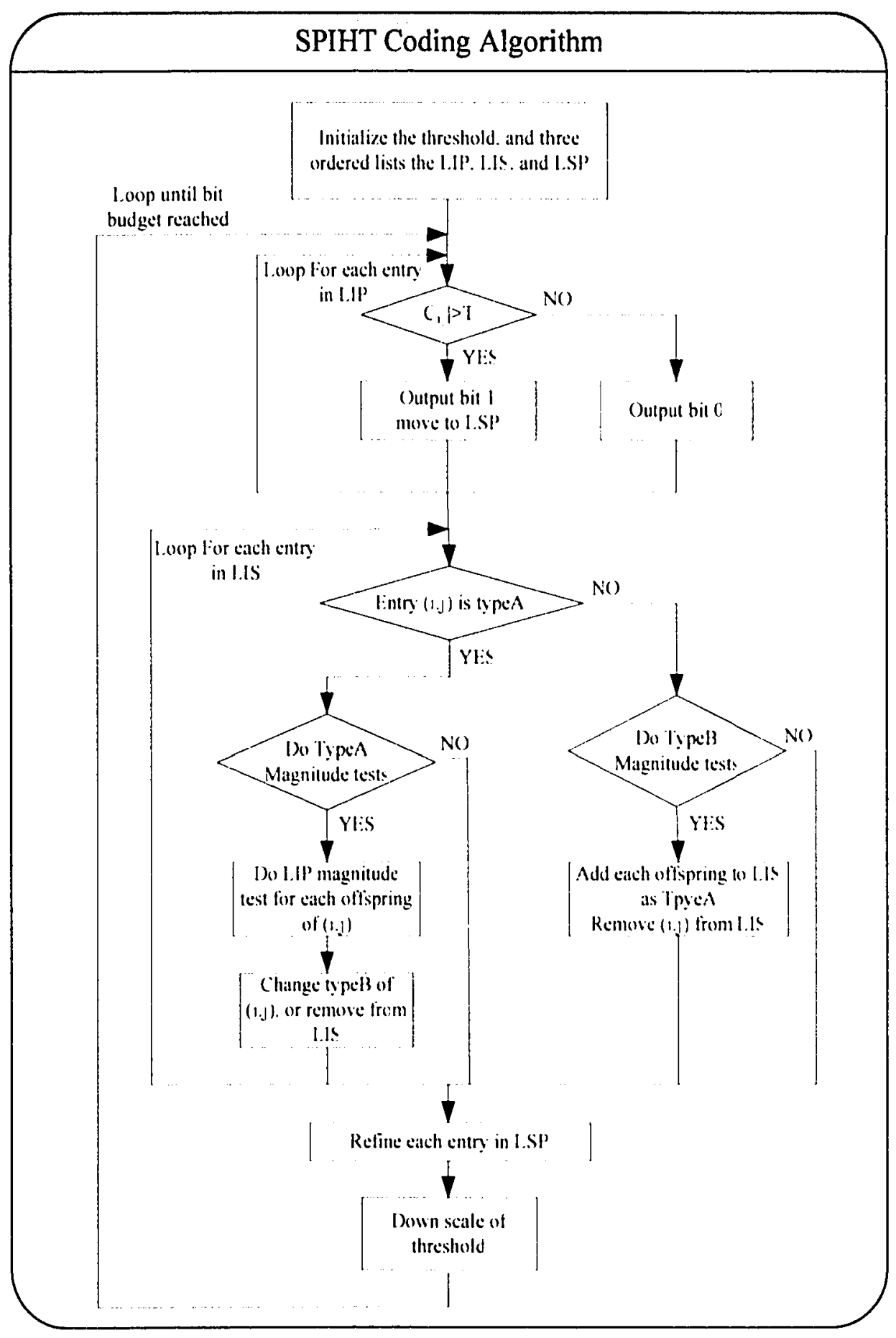

Figure 3.6 Flowchart of SPIHT algorithm 
1. In the sorting pass, the LIP is scanned to determine whether an entry is significant at the current threshold. If an entry is found to be significant, output a bit ' $l$ ' and another bit for the sign of the coefficient, which is marked by either ' 1 ' for positive or ' 0 ' for negative. Now the significant entry is moved to the LSP. If an entry in LIP is insignificant, a bit ' 0 ' is output.

2. Entries in LIS are processed. When an entry is the set of all descendants of a coefficient, named 'type $A$ ', magnitude tests for all descendants of the current entry are carried out to decide whether they are significant or not. If the entry is found to be as significant, the direct offsprings of the entry are undergone magnitude tests. If direct of fspring is significant, it is moved into LIP; otherwise it is moved into LSP. If the entry is deemed to be insignificant, this spatial orientation tree rooted by the current entry was a zero-tree, so a bit ' 0 ' is output and no further processing is needed. Finally, this entry is moved to the end of LIS as 'type $\mathrm{B}$ ', which stands for the set of all descendants except for the immediate offsprings of a coefficient, if there are still descendants of its direct offsprings. Alternatively, if the entry in LIS is type B, significance test is performed on the descendants of its direct offspring. If significance test is true, the spatial orientation tree with root of type B entry is split into four sub-trees that are rooted by the direct offspring and these direct offsprings are added in the end of LIS as type A entries. The important thing in LIS sorting is that entire sets of insignificant coefficients, zero-trees, are represented with a single 0 . The purpose 
behind defining spatial parent-children relationships is to increase the possibility of finding these zero-trees.

3. Finally, refinement pass is used to output the refinement bits ( ${ }^{\text {th }}$ bit) of the coefficients in LSP at current threshold. Before the algorithm proceeds to the next round, the current threshold is halved.

\subsubsection{JPEG2000}

JPEG2000 is the recent still image compression standard developed by Joint Photographic Experts Group (JPEG). JPEG2000 exploits intra-band dependency and adopts EBCOT algorithm [14] for coding bit planes of wavelet coefficients. The EBCOT algorithm, which is a context-based rather than tree-based method, offers excellent R-D performance and achieves spatial and distortion scalability with low complexity.

In EBCOT, each subband is partitioned into (fixed size) code blocks, which are numbered in raster scan order. Each code block is coded independently by employing fractional bit plane coding method and context based arithmetic coding. So a separate embedded bit stream is generated for each code block. R-D optimization over all code blocks is achieved by post-compression rate-distortion (PCRD) operation, which truncates each of the embedded bit streams of the code block to minimize the overall distortion subject to a given bitrate. PCRD optimization trades off rate-control flexibility to achieve optimal embedding. Below is a brief description of the bit plane coding 
primitives and coding passes of JPEG-2000. An overview of PCRD optimization method follows.

In each code block, EBCOT uses context-based arithmetic coding (CB-AC) to encode the bits for coefficients. Information to be encoded by $C B-A C$ can be classified into three cattegories:

- Significance information for each coefficient,

- Sign information for a significant coefficient,

- Refinement information if a coefficient is found to be significant at some bit plane level.

EBCOT first partitions each code block into sub-blocks, and employs quadtree coding to locate the sub-blocks which become significant at some bit plane level. In doing so, the number of bits to be processed by CB-AC is significantly decreased and the complexity reduced. Once a sub-block is found to be significant, the coefficients within that subblock are scanned according to fractional bit plane coding order, and each information bit is encoded by one of following four different bit coding schemes:

1. Zero Coding (ZC). The significant bits for coefficients are encoded with this primitive, based on the significance of their eight immediate neighbors.

2. Run-length Coding (RLC). If a run of four insignificant coefficients with insignificant neighbors is observed, the significance of this low activity region is 
represented by a single bit. A separate coding context is used to describe this bit.

3. Sign Coding (SC). This primitive is used to encode the sign information of a coefficient immediately after it is considered to be significant, by exploiting the signs of neighboring coefficients.

4. Magnitude Refinement (MR). This primitive is used to code next refinement bits for coefficients already identified as significant.

A coding method, called fractional bit plane coding, is employed to ensure fine cmbedding within a bit plane level. Each bit plane is processed in three kinds of coding passes, namely, Significance Pass, Refinement Pass and Cleanup Pass. To generate near optimal bit stream, a particular pass should be executed only if it results in the largest reduction in distortion for the smallest increase in bit rate. The coefficients that are predicted to be significant are encoded prior to other significance and refinement bits. For this reason, Significance Pass is executed before other passes. For each insignificant coefficient, the significance of its neighbors is tested. and insignificant coefficients with significant neighbors are encoded before magnitude refinement pass. Refinement pass is followed by cleanup pass which processes remaining (insignificant) bits and sign information for those coefficients that has not been processed during previous two passes.

PCRD Optimization is applied to minimize the distortion subject to a given bit rate over the bit streams of each code block. These bit streams are truncated in a certain way and efficiently interleaved to create a packet-stream. EBCOT adopts the convention of 
using ending points for each successive fractional bit plane coding phases as candidate truncation points.

During the bit plane coding, the slope of R-D curve is recorded for each fractional bit plane coding phase and this information is used to determine the feasible truncation points. The set of feasible truncation points is simply the subset of possible truncation points lying on the convex hull of the R-D curve. These points can be identified by employing convex hull analysis. Finally, the bit streams coming from each code block are interleaved according to the R-D slopes for each feasible truncation point, ordered from high to low R-D slope.

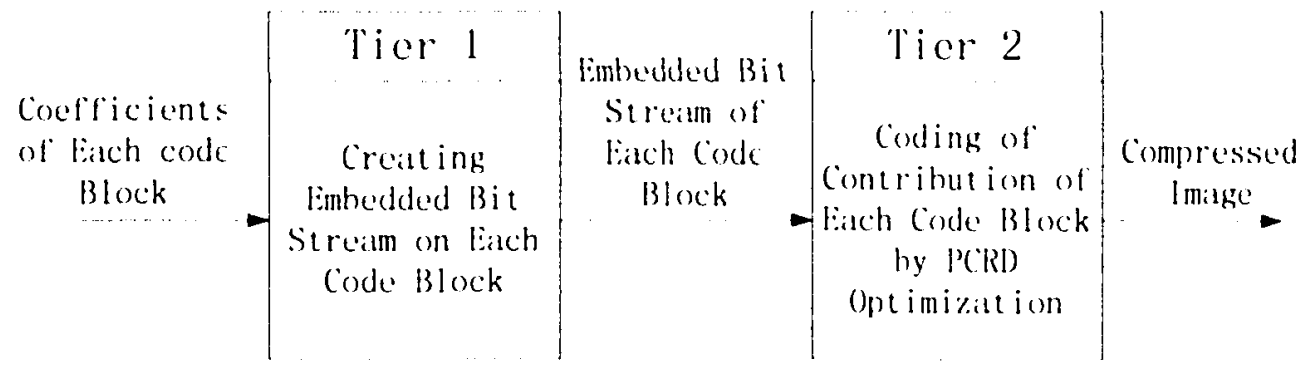

Figure 3.7 Two tier coding scheme employed in JPEG2000

In summary, the coding algorithm consists of two tiers as shown in Figure 3.7. In the first tier, a separate bit stream is obtained for each code block by bit plane coding modules while the (operational) R-D slopes for each fractional bit plane coding phase have been collected. The second tier analyzes the collected R-D curves of compressed bit streams and determines the contribution of each code block to the overall bit stream in 
the R-D optimal sense. This two-tiered scheme results in excellent R-D performance with low computational cost. However, since the optimization is performed after collecting the compressed representations of all code blocks, the precise rate control is traded off to PCRD optimization.

\subsection{ROI Coding}

Region-of-interest (ROI) coding is an important feature in current image compression codees, which allows different regions of an image to be compressed at different qualities. ROI coding provides a way to encode the significant regions in an image at higher precisions (up to lossless) than the background. Especially in medical imaging. ROI coding can help compression methods to focus on those regions that are important for diagnostic purposes [8] [23]. SPIHT and JPEG2000 both support ROI coding but they use different techniques to incorporate this feature.

\subsubsection{ROI Coding in JPEG2000}

In JPEG2000 codec, in order to encode region-of-interest with higher quality than the remaining, i.c. background, at a certain bit rate constraint, the algorithm need to identify the wavelet coefficients that are involved in the reconstruction of the pixels in the ROI.

JPEG2000 uses region mapping which trace the positions of pixels in an ROI in image domain back into transform domain by inverse DWT. Starting from linal 
reconstruction level, the sets of locations of coefficients which will be used to restore the ROI pixels can be simply found by single inverse DWT stage. By progressively tracing back until final level of inverse DWT, the sets of coefficients involved in reconstruction of the ROI are determined. Thus, some of the transform coefficients are identified as more important for ROI coding and the remaining coefficients are treated as irrelevant to the reconstruction of the ROI. The coefficients of greater importance are called ROI coefficients, the rest are called background coefficients.

ROI coding mechanism is applied in the tier-1 coding stage of JPEG2000. Before bit plane coding is performed, ROI coefficients are scaled up by a number of a power of 2 . Such scaling up can be easily implemented by left bit shifting. Suppose that the background coefticients have the largest magnitude with most significant bit (MSB) at $\mathrm{N}$ 1. There are two methods for performing the scaling up operation. One is left shifting of ROI coefficients with an integer number between 1 and $N-1$. Doing so is equivalent to assigning priority to the ROI coefficients; hence more bits will be used to code the ROI coefficients than the background coefficients. Alternatively, one can perform left shifting on all ROI coefficients with $\mathrm{N}$ bits, called Maxshift, such that all ROI coefficients lie in the bit plane more significant than the background coefficients. Maxshift method can be viewed as a specific case of scaling method. As a consequence, all information about the ROI is coded before the background coefficients. Figure 3.8 illustrates scaling and Maxshift methods. 


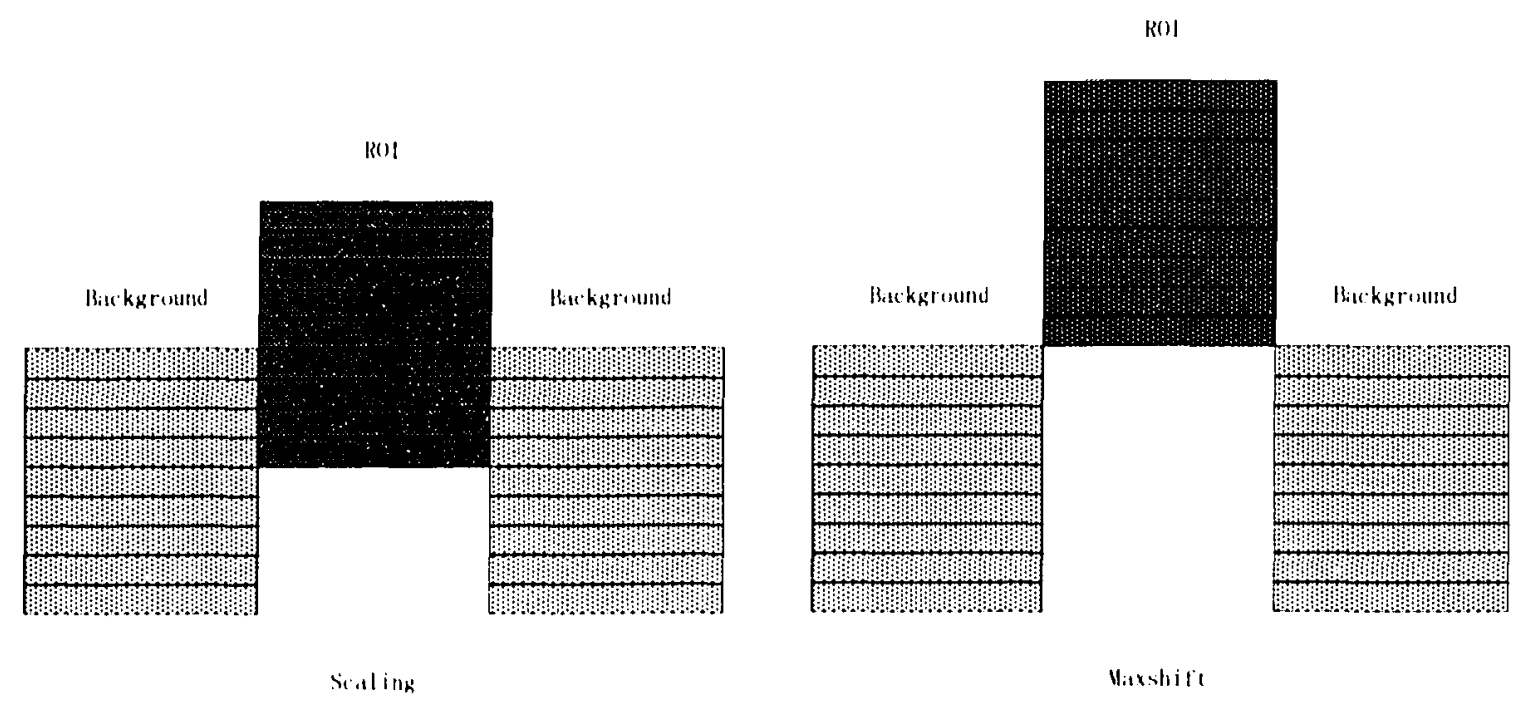

Figure 3.8 Scaling and Maxshift methods used in JPEG2000

\subsubsection{ROI Coding in SPIHT}

In contrast to JPEG2000 which uses conventional wavelet transform and scaling method for ROI coding, ROI-SPIHT employs shape-adaptive discrete wavelet transform, previously discussed in the beginning of this chapter, for wavelet decomposition of an image containing and ROI.

Recall that SA-DWT keeps the number of transform coefficients which are related to reconstruction of ROI (ROI coefficients) the same as the number of pixels in the ROI of the image. Also important features such as spatial correlation, locality and self-similarity across subbands are all well preserved in the SA-DWT [3]. The conventional SPIHT coding algorithm can be used in coding of ROI coefficients with a modification. The coding algorithm needs to keep track of the locations of wavelet coefficients according to 
the shape of the ROI. To obtain the information about ROI coefficients, a mask image which specifies the ROI is decomposed by the same SA-DWT used in the wavelet decomposition of the image. In each decomposition stage, each subband of the decomposed mask contains information for specifying the ROI in that subband. By successively decomposing the approximation coefficients (LL subband) for a number of decomposition levels, information about ROI coefficients is obtained.

Once the ROI coefficients are identified, SPIHT coding algorithm will applied on these coefficients to create the embedded bit stream. In order to efficiently code ROI coefficients by taking advantage of the ROI information in the transform domain, an object-based extension of SPIHT algorithm is used. When the spatial orientation trees are established in the initialization step of SPIHT, ROI information obtained from decomposition of the ROI mask is used to mark the spatial orientation tree. If all coefficients or some coefficients in a spatial orientation tree belong to the ROI, the corresponding tree is marked as an ROI tree. If all coefficients in a tree are outside the ROI, this tree is identified as a background tree. The background tree is skipped at the initialization stage. Also when a node and all its descendants in a spatial orientation tree are outside the ROI, the tree is pruned from that node. By doing so, no information about coefficients outside the ROI needs to be coded. Figure 3.8 gives an example of the parent-children relationship after SA-DWT decomposition of an image containing a ROI. 


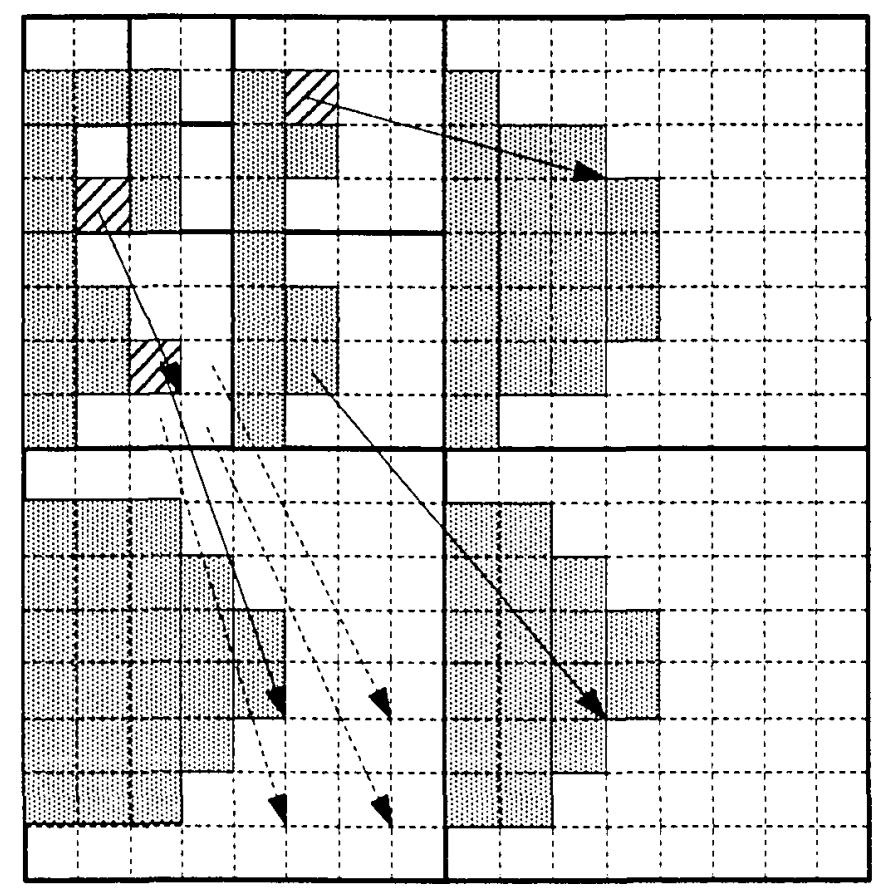

Figure 3.9 Parent-children relationship in SA-DWT subbands

In Figure 3.9, shaded blocks represent ROI coefficients, and striped blocks are the background coefficients that have descendants inside the ROI. Solid arrows represent the parent-children relationship in the coefficient tree that should be kept. Dashed arrows specify the sub-branches in the coefficient tree that can be pruned. Because there are no ROI coefficients in these sub-branches, keeping these sub-branches will not improve the performance of coding.

\subsection{Summary}

In this chapter, we reviewed the shape adaptive discrete wavelet transform used for ROI image coding. The spatial correlation, locality of wavelet transform and self-similarity 
across subbands are well preserved by SA-DWT. Then, two state-of-the-art bit plane coding algorithms, SPIHT and JPEG2000, are described. SPIHT algorithm exploits interscale dependencies of coefficients to encode subbands, and JPEG2000 makes use of intra-scale dependencies between coefficients. Finally, the ROI coding methods used in SPIHT and JPEG2000 are discussed. A SA-DWT is used in ROI-SPIHT and MAXSHIFT method is adopted in JPEG2000 for ROI coding. In the following, we present the implementation of 3D SPIHT coding algorithm and the corresponding ROI coding method. 


\section{Chapter 4}

\section{SPIHT-Based Three-Dimensional Image}

\section{Coding}

In this chapter, we present three-dimensional extension of the original SPIHT coding algorithm. Due to its success in still image compression, it is reasonable to extend the 2D SPIHT to three dimensions and expect to obtain good R-D performance for the compression of volumetric datasets and video sequences. The main objective in applying three-dimensional coding schemes is to exploit the correlations between image pixels across the third dimension (inter-slice or temporal direction) to achieve better compression ratio.

In section 4.1, an overview of three-dimensional SPIHT (3D SPIHT) coding algorithm is presented. In section 4.2, three-dimensional SPIHT coding algorithm using dyadic and packet wavelet transforms are discussed. A relevant technique based on 3D SPIHT using optimal coefficient tree structure is discussed in section 4.4. Finally, 3D SPIHT ROI coding method, based on unbalanced tree structure is presented. 


\subsection{Three-Dimensional SPIHT}

3D SPIHT coding scheme is developed by Kim et.al. in [7] for video coding. This algorithm is later used to compress volumetric medical image data by Xiong et al in [8]. A new method based on optimal 3D coefficient tree structure proposed for video coding in [6], establishes 3D coefficient trees according to the relationships of coefficients in the spatial and temporal dimensions.

3D SPIHT coding scheme is extended from 2D SPIHT algorithm. Figure 4.1 shows a general scheme for the 3D SPIHT coder.

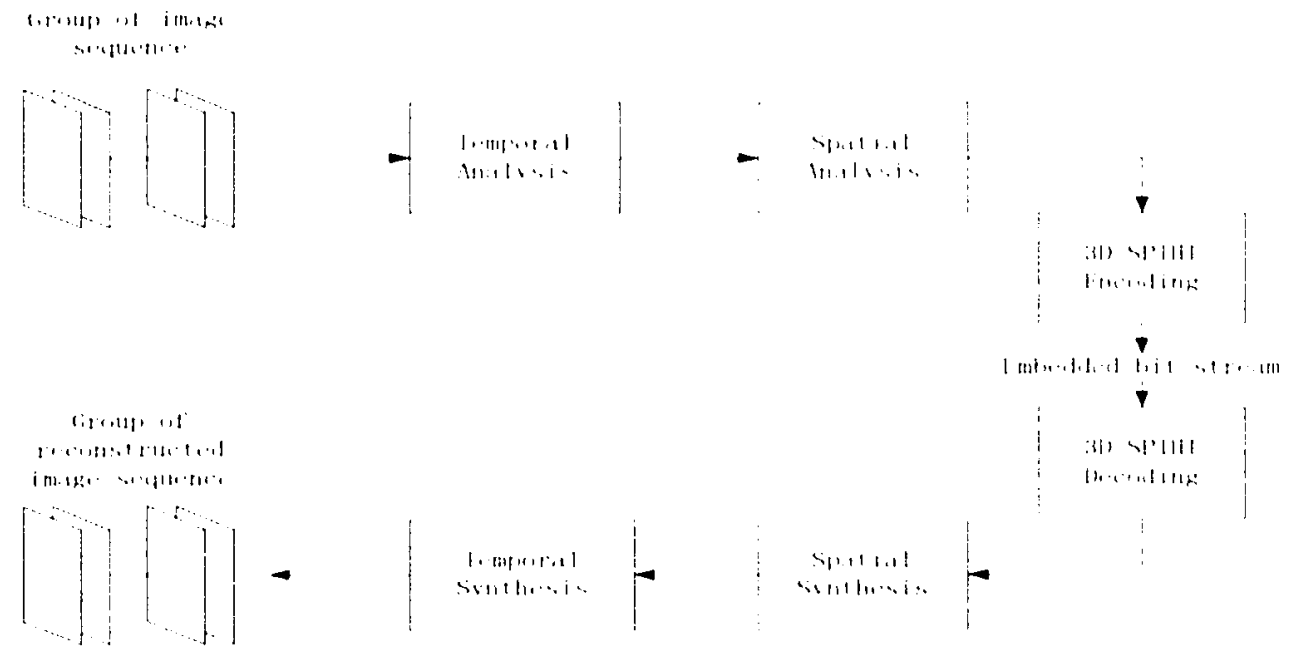

Figure 4.1 3D SPIHT coding scheme

An image sequence is typically divided into a group of frames/slices (in video, each image in the sequence is called a frame; in volumetric datasets, each image in the volume is called a slice), called GOF/GOS. The length of the GOS/GOF size has an immediate 
effect on the compression algorithm. Large GOF/GOS sizes results in better compression ratios but require more memory both at the encoder and the decoder and causes coding delays [6] [9]. If the size of GOF/GOS is small, the boundary effect becomes significant, that is the PSNR drops severely at the GOF/GOS boundaries [4]. This can degrade the overall coding performance. Typically, every 16 or 32 frames [6] [9] [10] are grouped together in video compression and every 64 or 128 frames (if available) are put together for compression of volumetric data since decoding speed is not a major concern [2] [4].

3D dataset is decomposed using three-dimensional wavelet transform into threedimensional subbands, then modified SPIHT coding algorithm is used to create compressed bit stream using bit plane coding. 3D SPIHT decoder extracts information from the bit stream to create the reconstructed image sequence. Recall that the compressed bit stream is embedded and scalable by quality. Wavelet transform on threedimensional data can be carried out by calculating one-dimensional transform along the temporal dimension and then applying the two-dimensional spatial transform on each resulting frame. At the next decomposition level, wavelet transform is applied on the lowest subband and this process repeated in a cascaded manner, until desired number of levels is achieved. Alternatively 3D wavelet transform can be calculated by first performing one-dimensional transform along the temporal dimension until the required number of temporal decomposition levels is obtained and then carrying out the twodimensional transform on each temporal-transformed frame up to the desired number of spatial decomposition levels [9]. 


\subsubsection{D-SPIHT Coding using Dyadic Wavelet Transform}

In order to take the advantage of the dependencies of the neighboring pixels between the frames in an image sequence, the wavelet transform should be applied on image sequence along temporal dimension in addition to two-dimensional spatial decomposition on each frame or slice. By doing so, signal energy will be compacted in low frequency subbands of transformed frame.

Similar to two-dimensional spatial decomposition, three-dimensional dyadic decomposition scheme applies temporal and spatial wavelet transform separately and alternately. Figure 4.2 gives an example that illustrates a three level $3 \mathrm{D}$ dyadic decomposition.
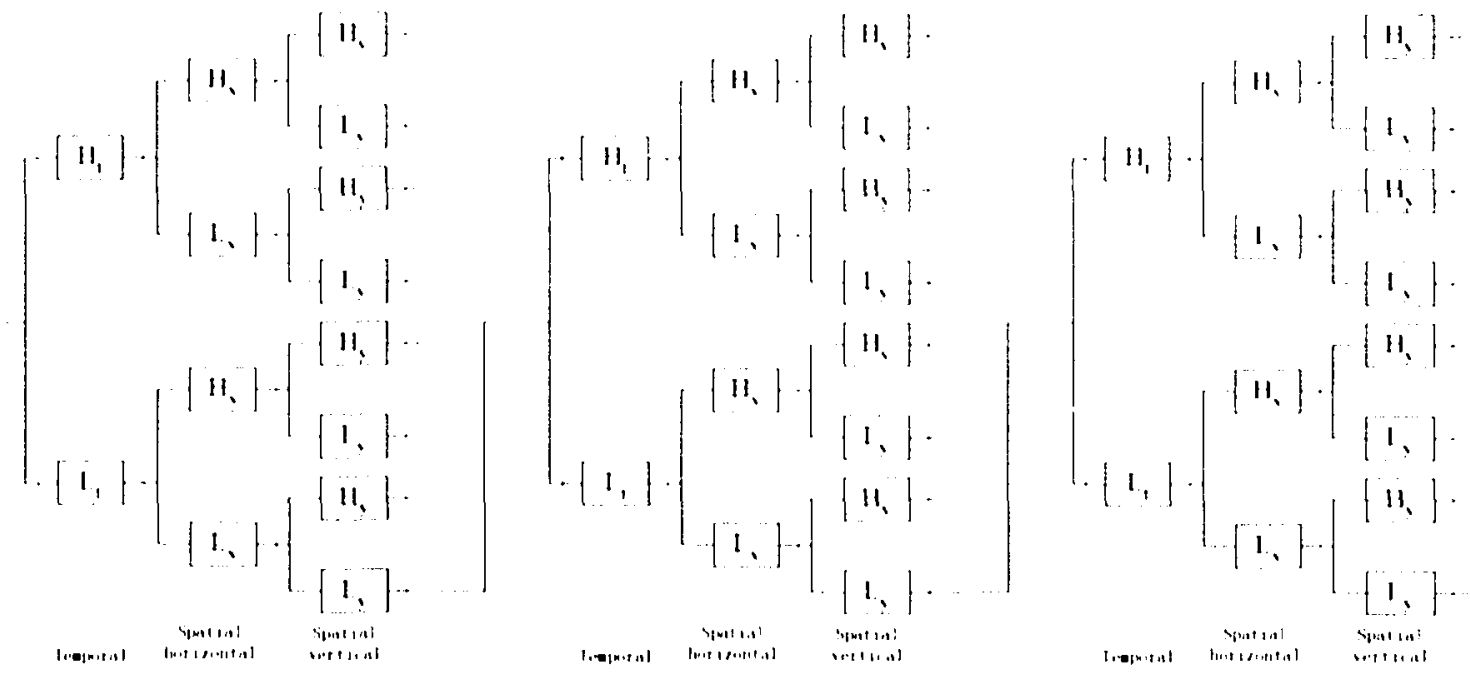

Figure 4.2 Three-levels 3D dyadic decomposition 
First, ID wavelet transform is applied on the whole data sequence along temporal dimension. The sequence is decomposed into low frequency and high frequency subbands. For the low and high frequency subbands, conventional 2D spatial decomposition is carried out to obtain eight subbands. Then, the decomposition process is repeated on the lowest frequency subband obtained at the previous stage until required number of decomposition levels is obtained. Thus, a three dimensional dyadic subband structure is obtained. As can be seen, there are a total of twenty three subbands in Figure 4.2.

In a two-dimensional dyadic wavelet transform in Figure 3.5, each coefficient is related to a set of coefficients in the next higher level subband, called coarser subband. which corresponds to the same spatial location in the subband pyramid. A coefficient in lower frequency subband is called the parent coefficient, and its corresponding coefficients in coarser subband are called children coefficients. Recall that children coefficients have a $2 \times 2$ group form in the same subband. All the coefficients descending from a coefficient are referred as descendants of this coefficient.

For three-dimensional dyadic wavelet transform, the parent-children relationship among the coefficients in the subband pyramid can be derived based on the relationship defined for two-dimensional case. A coeflicient (i, j, k) has 8 children coefficients that are grouped in the form of a $2 \times 2 \times 2$ cube in the liner level subband. These children coefficients are defined as given below: 


$$
\text { children }=\left\{\begin{array}{c}
(2 i, 2 j, 2 k),(2 i+1,2 j, 2 k),(2 i, 2 j+1,2 k),(2 i, 2 j, 2 k+1) \\
(2 i+1,2 j+1,2 k),(2 i+1,2 j, 2 k+1),(2 i, 2 j+1,2 k+1),(2 i+1,2 j+1,2 k+1)
\end{array}\right\}
$$

For a coefficient in the lowest frequency subband, it either has no children or has 8 children. Obviously, the coefficients in the highest frequency subband have no children. Figure 4.3 illustrates the parent-children relationship in three dimensions.

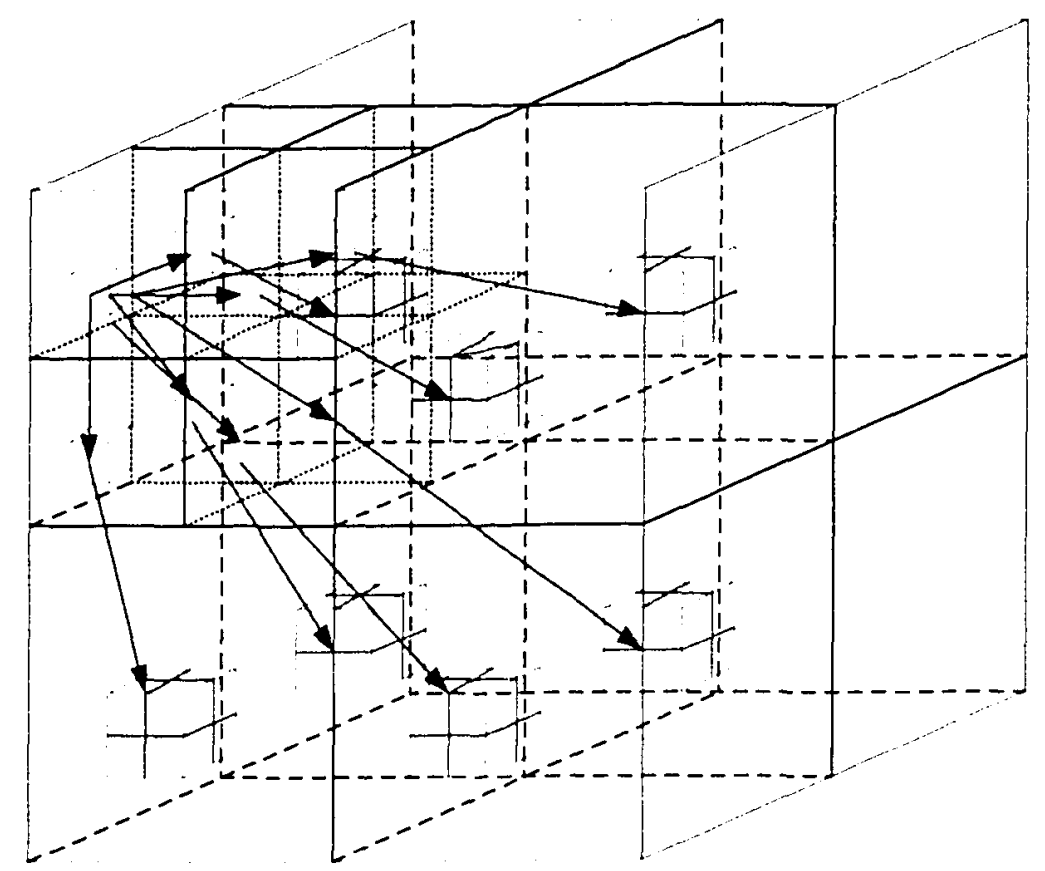

Figure 4.3 Parent-children relationship in three dimensions

After three-dimensional spatial-temporal coefficient trees are established, 3D SPIHT coding algorithm is used to sort these coefficients. Three-dimensional SPIHT coding algorithm can be developed by modifying its two-dimensional counterpart. 
Since the image sequence is decomposed by three dimensional wavelet transform, the energy of image sequence is compacted into lower frequency subbands of three dimensional decomposition pyramid structure. In fact, 3D SPIHT coding scheme exhibits the same characteristics as 2D SPIHT such as ordering of the magnitude of transformed coefficients, ordered bit plane coding, and exploitation of the dependency between parent and children coefficients in spatial-temporal orientation trees. The compressed bit stream is still embedded completely, thus progressive decoding of this single bit stream can provide reconstruction of image sequence at different quality levels.

\subsubsection{Three-Dimensional SPIHT Coding using Wavelet Packet Transform}

A problem with three dimensional dyadic decompositions of image sequence is that the number of decomposition levels and the wavelet used in the transform should be the same on both spatial and temporal dimensions. De-coupled wavelet transform, also called packet wavelet transform, solves this problem by decoupling the spatial and temporal decompositions. An example of 3 temporal decomposition levels and 3 spatial decomposition levels using packet wavelet is illustrated in Figure 4.4. 


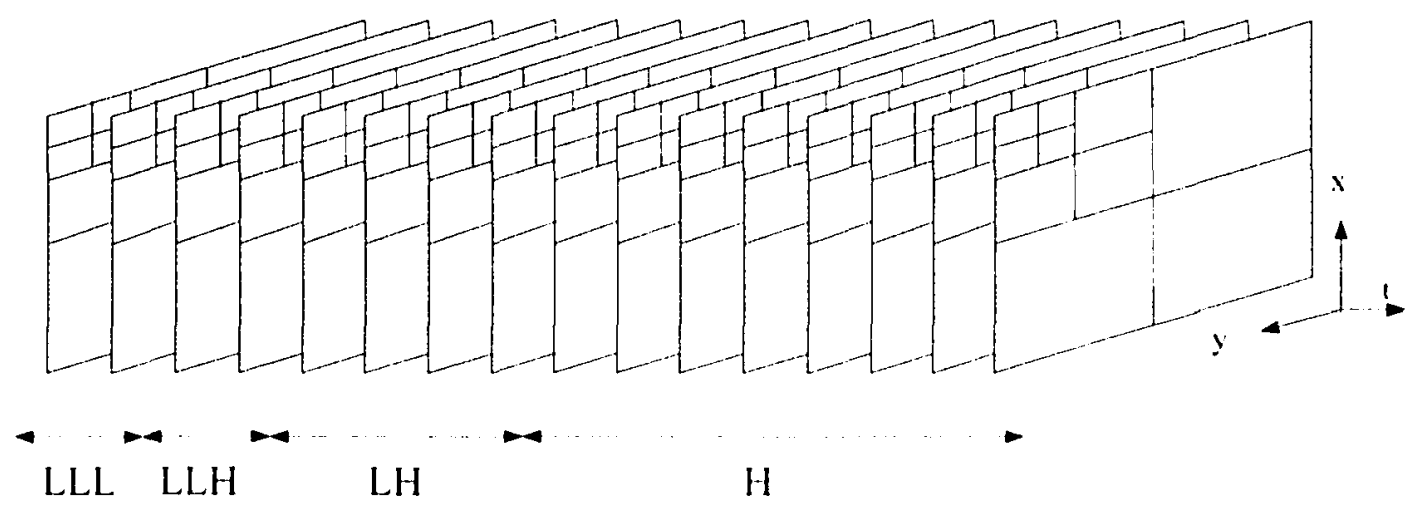

Figure 4.4 Three-dimensional packet decomposition

As shown in Figure 4.4. first three levels of temporal decompositions are applied on the image sequence. Then three levels of spatial decompositions are carried out on each temporal transformed frame; in total forty subbands are obtained. Using this scheme, different number of decompositions along spatial and temporal dimensions is allowed. Also, different wavelet transforms can be applied on spatial and temporal dimensions to exploit different characteristics of these dimensions. Clearly, packet transform provides more flexible decomposition mechanism than dyadic transform.

In [10] and [34] the symmetric spatial-temporal orientation trees used in dyadic decomposition scheme is adopted. Figure 4.5 shows this spatial-temporal orientation tree structure used in packet decomposition. It is argued that when the symmetric tree structure is used for packet decomposition, the coefficients in the spatial-temporal orientation tree may no longer be related to the same location because the subbands obtained as a result of packet transform are no longer symmetric. For example, in Figure 
4.6, $\mathrm{C}$ represents the children of $\mathrm{B}$ but $\mathrm{B}$ corresponds to the middle frame of GOF. $\mathrm{C}$ is related to the end and beginning frame of the GOF. To solve this problem, a packet tree structure is used in [9]. Figure 4.6 illustrates the packet tree structure.

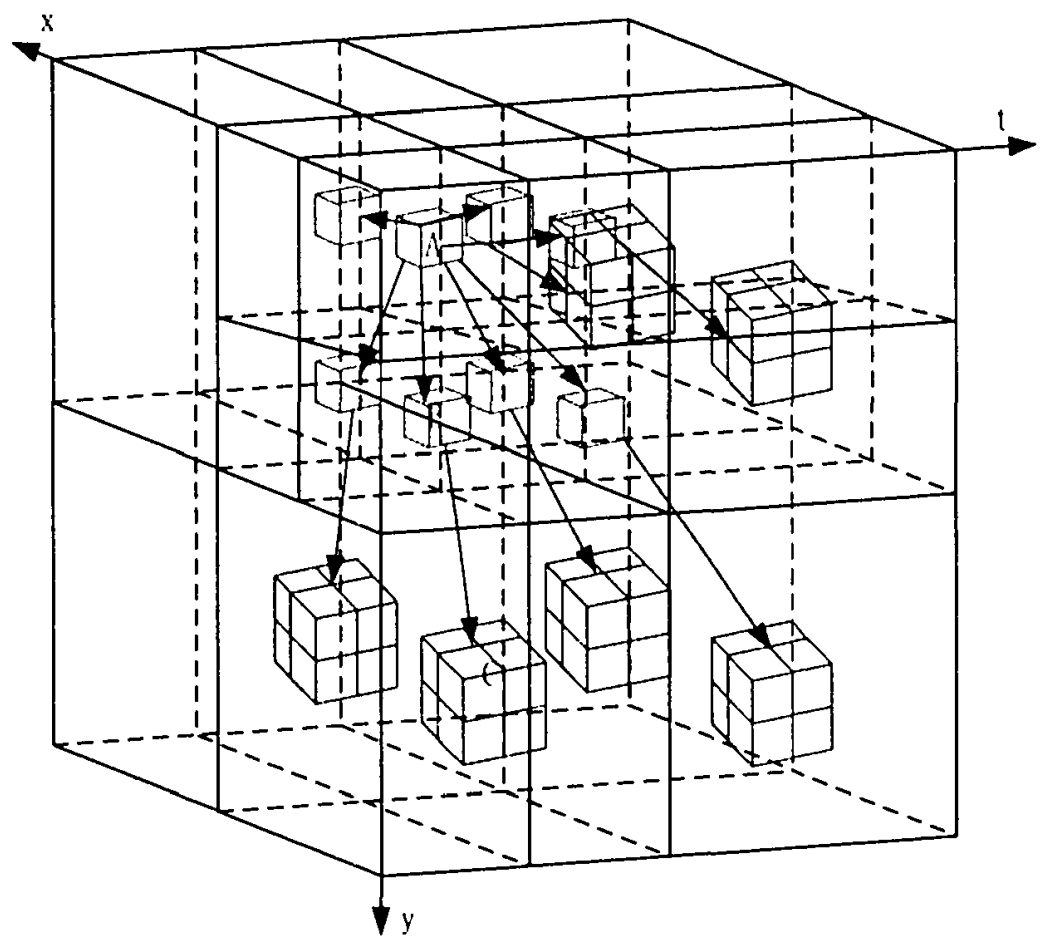

Figure 4.5 Symmetric tree structure in packet wavelet decomposition 


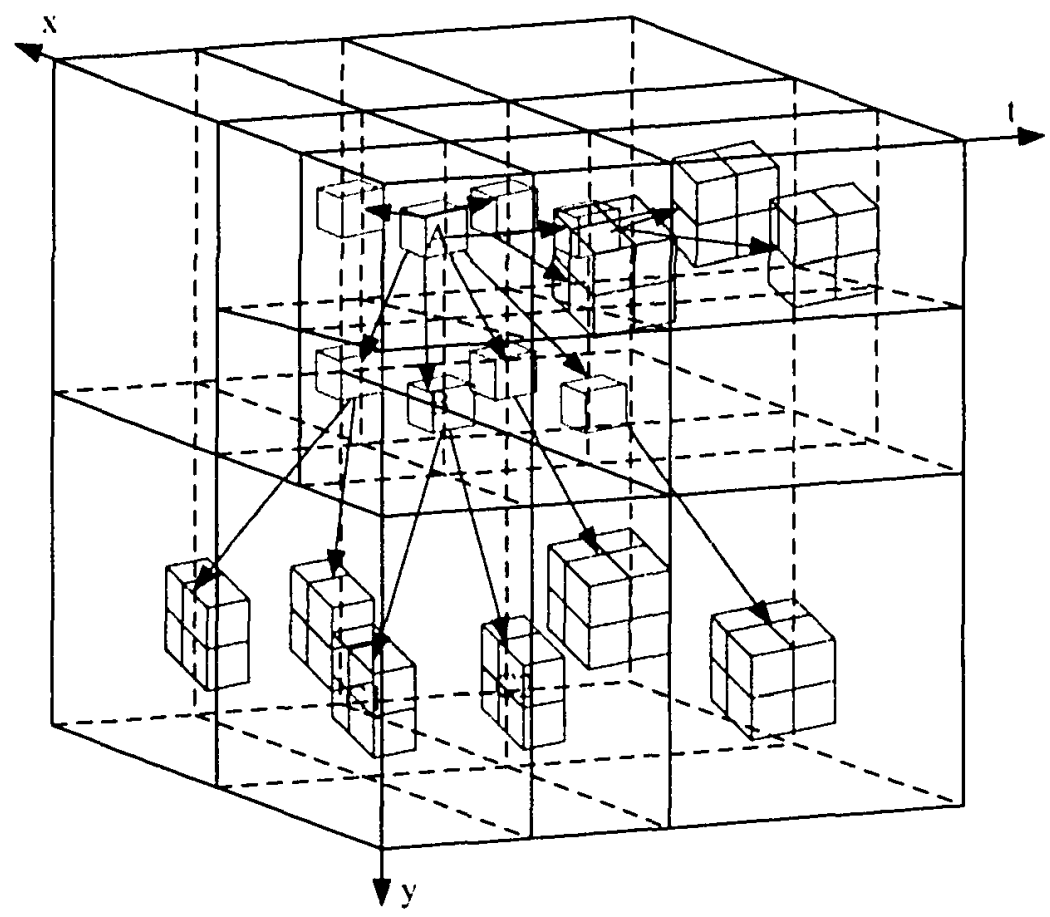

Figure 4.6 Packet tree structure in packet wavelet decomposition

\subsection{Optimal 3D Coefficient Tree Algorithm}

A new spatio-temporal orientation tree structure, called optimal 3D coefficient tree structure, is proposed in [6] to create the coefficient trees for compression of video. Optimal 3D coefficient tree exploits the dependency among subbands to make the coefficient trees longer so that coefficients in these trees exhibit spatial correlation and are of better zero clustering than in symmetric trees or packet trees. The optimal 3D coefficient tree structure is illustrated in Figure 4.7. 


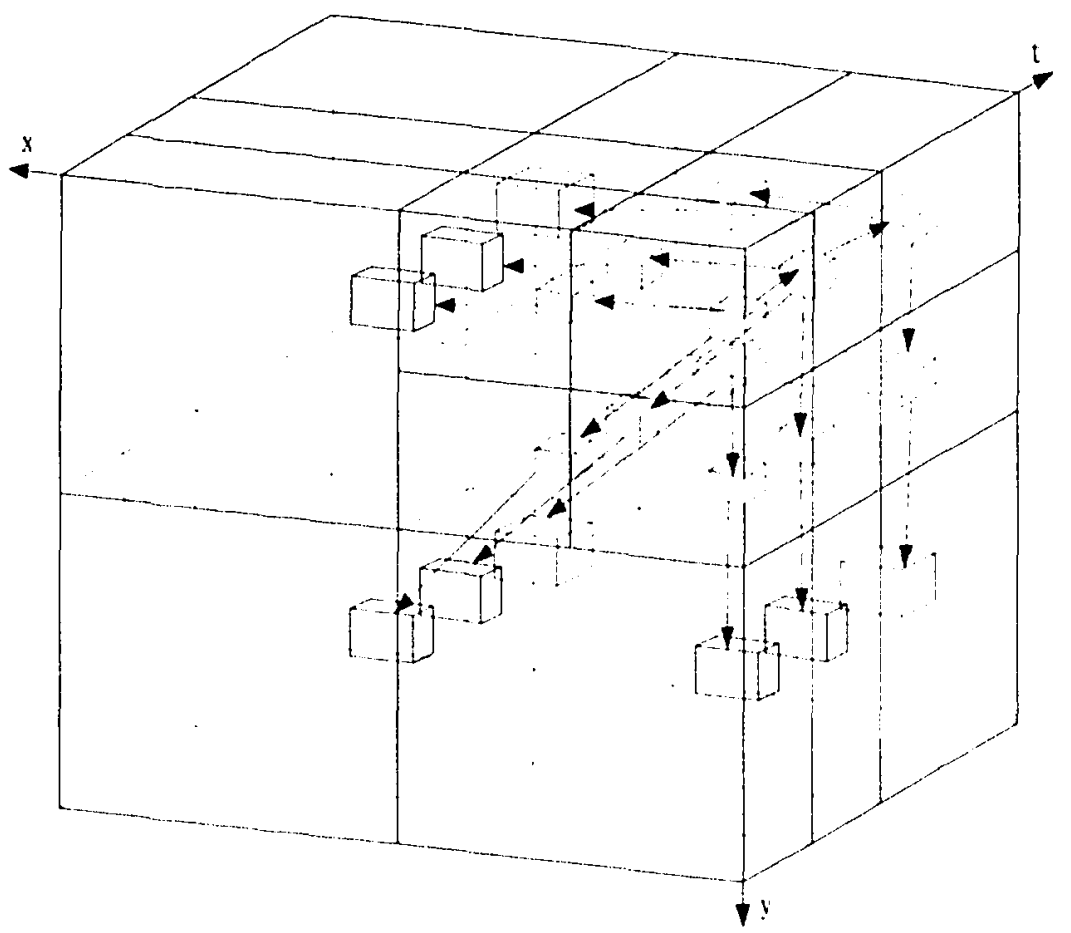

Figure 4.7 Optimal 3D coefficient tree structure

Assuming Fs, Rs, Cs are the frame size, row size and column size of the lowest frequency wavelet subband respectively, the children coefficients of a coefficient at frame $i$, row $j$, and column $\mathrm{k}$ in wavelet domain are determined according to the rules given below:

1. If coefficient (i, $j, k$ ) is located in the lowest frequency subband, its children coefficients consists of 4 coefficients $\{(i, j+R s, k),(i, j, k+C s),(i, j+R s, k+C s),(i+F s, j$, k)\}. 
2. If coefficient $(i, j, k)$ is located in the spatial lowest frequency subband except in the case 1 , it has 5 children coefficients with the following coordinates $\{(i, j+R s, k),(i, j$, $k+C s),(i, j+R s, k+C s),(2 i, j, k),(2 i+l, j, k)\}$.

3. If coefficient ( $i, j, k$ ) does not belong to any of the previous two categories, it has 4 children coefficients $\{(i, 2 j, 2 k),(i, 2 j+1,2 k),(i, 2 j, 2 k+1),(i, 2 j+1,2 k+1)\}$, as in $2 D$ wavelet transform.

Optimal 3D tree structure is not symmetric but it is consistent with 3D wavelet transform structure. Thus, the dependencies between coefficients in the optimal orientation tree are strong and coding method based on it outperforms the symmetric and symmetric-alike methods that employ conventional tree structure [6].

\subsection{Unbalanced Tree Algorithm for 3D ROI Coding}

In previous chapter, ROI coding algorithms for still images were presented. These coding methods can also be extended to coding of three-dimensional ROl of image sequences. In [1] and [25], a 2D unbalanced tree structure is introduced to construct the coefficient trees for ROI coding. Labels are used to differentiate ROI coefficients from background coefficients. Then, unbalanced orientation trees are constructed according to the label information. If the label of a coefficient in a tree does not agree with the label of its parent coefficient, parent coefficient is said to be pseudo-parent, and in this case, the 
coefficient is disconnected from its pseudo-parent and a new (real) parent coefficient is sought for this coefficient. The real parent is defined as one of the eight-connected neighbors of the pseudo-parent coefficient and has the same label as the corresponding coefficient. If such a parent coefficient is found, the coefficient is connected to its real parent coefficient; otherwise the coefficient itself is used as a zerotree root upon LIS initialization.

Similar to two-dimensional case, after the computation of 3D SA-DWT the spatiotemporal orientation trees are constructed. These coefficient trees are classified into three categories: 1) All nodes in the tree are inside the wavelet domain ROIs and they belong to the ROIs; 2) All nodes in the tree are outside the ROIs and contain no information about ROI; 3) Some nodes in the tree are outside the ROIs, and some inside the ROls. Thus, parts of the coefficient trees contain useful information to improve the R-D performance of the coding algorithm. First two types of trees are easily treated just by keeping the first and skipping the second as in conventional SPIHT method. For the $3^{\text {rd }}$ type of coefficient tree, if a coefficient in the tree is outside the ROIs and all of its descendant coefficients are outside the ROIs as well, the branch from this coefficient is pruned from the ROI coefficient tree. If a coefficient in the tree is inside the ROIs and its parent coefficient is outside the ROIs, called pseudo-parent, the coefficient is disconnected from its pseudoparent. A new parent, which is inside the ROIs, is searched within the subband of its parent. The searching process is performed from the nearest coefficients to its farthest coefficients of its pseudo-parent. If new parent is found, called the real-parent, that 
coefficient is connected to its real-parent. After reconnection, there are only two kinds of coefficient trees left. One is with all coefficients in the tree inside the ROls, the other with all coefficients in the tree outside ROIs. The coefficient trees are not balanced any longer. The algorithm to construct unbalineed coefficient trees is described in Figure 4.8.

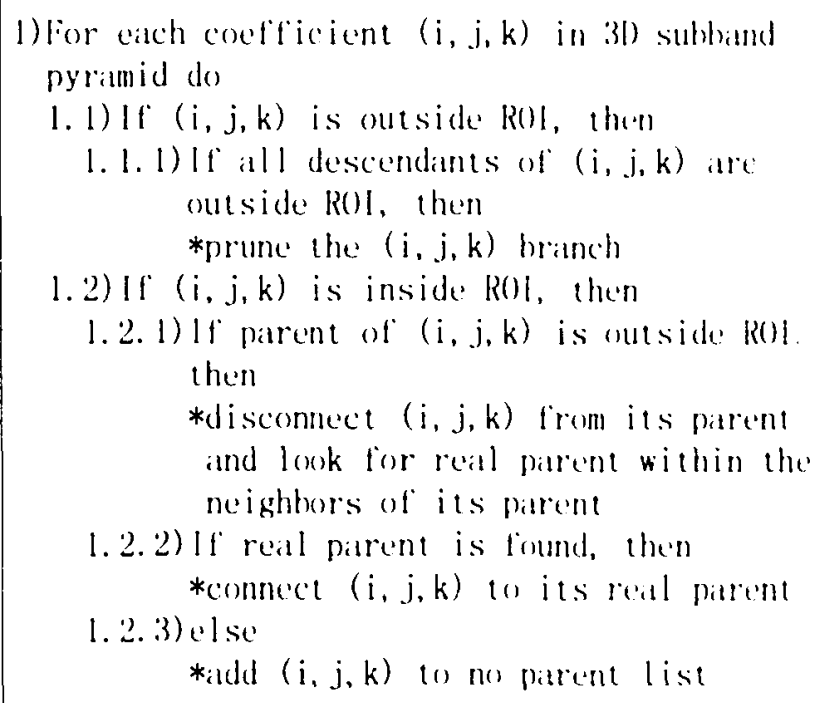

Figure 4.8 Algorithm to create unbalanced trees

Thus new tree structure is applied and access to the descendents of a coefficient should be modified along the unbalanced trees. A datta structure, a list named LNP, is used to collect the isolated coefficients for which no parents in the lowest subband are found. The modified ROI SPIHT algorithm places the coefficients both in the lowest subband and inside ROIs into LIP or LIS, and the coefficients in LNP are also appended to LIP or LIS. These coefficients, which are in the lowest subband but outside ROls, are skipped when SPIHT algorithm is initialized. 
The sorting pass is modified using unbalanced trees structure to get the descendents of a coefficient and refinement pass in our algorithm is the same as original SPIHT. With this method, coefficients not belonging to the ROIs are not included in the corresponding trees through the sorting pass procedure in SPIHT algorithm. The modified ROI 3D SPIHT coding algorithm based on unbalanced tree is given in Figure 4.9.

\subsection{Summary}

In this chapter, we reviewed three-dimensional wavelet transforms: dyadic and packet wavelet transforms. Conventional 3D spatio-temporal orientation tree structure and optimal coefficient tree structure are presented. Finally, a modified 3D SPIHT ROI coding method, based on unbalanced tree structure, extended from its 2D version is proposed. 


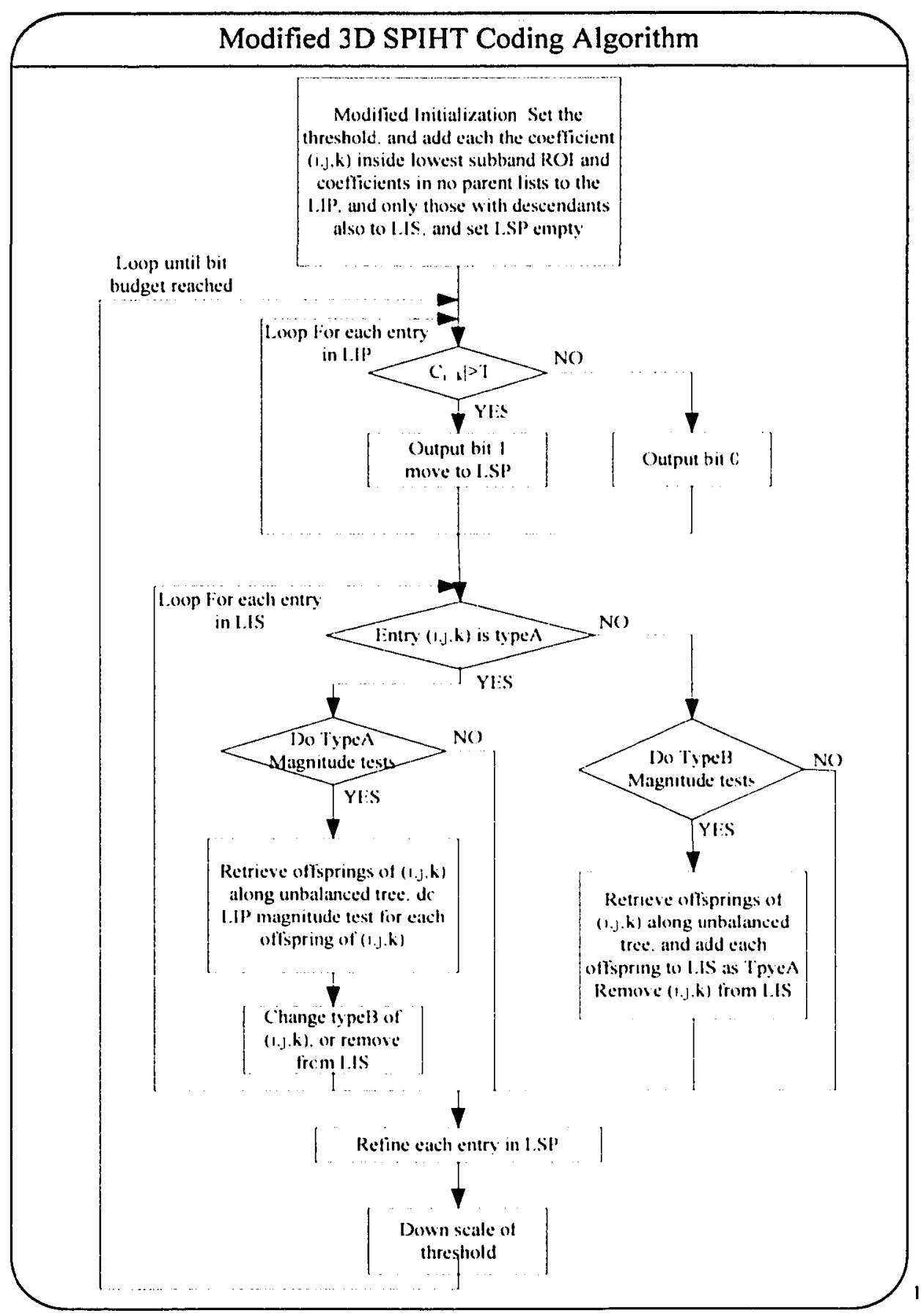

Figure 4.9 Modified 3D SPIHT algorithm based on unbalanced trees

' TypeA and TypeB are defined in Chapter 3, on page 34. 


\section{Chapter 5}

\section{Three-Dimensional MRMQ Coding}

In previous chapter, 3D ROI SPIHT coding algorithm based on unbalanced tree structure was presented. For compression of volumetric datasets, multiple ROI's selected by the user are to be encoded in 3-D with higher quality than the rest of the image. In this chapter, we present a 3D extension of a previously proposed ROI-based coding technique, called Multiple-region Multiple-quality coding algorithm.

The content of this chapter is organized as follows. In section 5.1, the wavelet representation for multiple ROls is presented. Then, 3D MRMQ coding algorithm is presented and 3D MRMQ-SPIHT coding method based on unbalanced tree structure is described in section 5.2. Finally, in section 5.3, we present the construction of a bit stream structure for coding of multiple ROIs at multiple quality levels.

\subsection{Wavelet Representation for Multiple ROIs}

In MRMQ coding, the goal is to compress the different regions of image sequence according to different specified quality constraints. Typically, a number of frames or slices of image sequence are grouped into a volume, $\mathrm{V}$ and this volume is partitioned into 
$K$ disjoint sub-volumes, $V_{i}\{i=0, \ldots, K-1\}$. Clearly the union of these subvolumes constitutes the entire group of image sequence, $V$. When a wavelet transform is applied on the partitioned image volume, each sub-volume is decomposed separately into wavelet coefficients in the transform domain. In other words, the pixels within each subvolume $V_{i}$ are mapped onto a set of volumes of wavelet coefficients defined over $\Delta_{i}$ by wavelet decomposition. Also all pixels of entire volume are transformed to wavelet coefficients over a volume $\Delta$ in transform domain. Thus, we can express this relation as:

$$
\Delta_{1} \subseteq \Delta
$$

When multiple regions of image sequence are decomposed by conventional wavelet transform, it is highly likely that [25]

$$
\Delta_{1} \cap \Delta, \neq \varnothing \text {, if } i \neq j
$$

That is, conventional wavelet transform does not ensure that wavelet representations of multiple disjoint regions will be completely separated in the transform domain. Some coefficients may contribute to several different regions during the reconstruction process. In this case, it is difficult to encode these coeflicients of multiple regions separately at different specified quality levels. However, recall the discussion in chapter 3, SA-DWT can keep the number of coefficients in transformed ROI the same as the number of pixels in image ROI. Using SA-DWT, it is possible to make volumetric subsets $\Delta_{i}$ 's mutually 
disjoint. All volumetric subsets $\Delta_{i}$ 's that make up the entire transform volume is expressed as:

$$
\Delta_{i} \cap \Delta_{j}=\varnothing \text { and } \bigcup_{i=0}^{K-1} \Delta_{i}=\Delta
$$

As mentioned in chapter 3, odd symmetric biorthogonal wavelet (OS-BW) basis will be used in 3D SA-DWT for decomposition of multiple volumetric ROIs with symmetric extension. For odd symmetric biorthogonal wavelet, we use lowpass filter centered at even positions and highpass filter centered at odd positions. To perform 3D SA-DWT, OS-BW is used to filter each dimension of image sequence. After filtering ID signals with OS-BW, the output is subsampled by a factor of 2 . For signal segment starting from even positions, even subsampling method is used for lowpass-filtered coefficients and odd subsampling method is applied to highpass-filtered coefficients. Odd subsampling for lowpass-filtered coefficients and even subsampling for highpass-filtered coefficients are chosen if the signal segment starts from odd positions. These subsampling strategies help us to obtain transform representations of multiple regions that satisfy the conditions given in equation 5.3. The aformentioned coefficient subsampling process illustrated in Figure 5.1. 


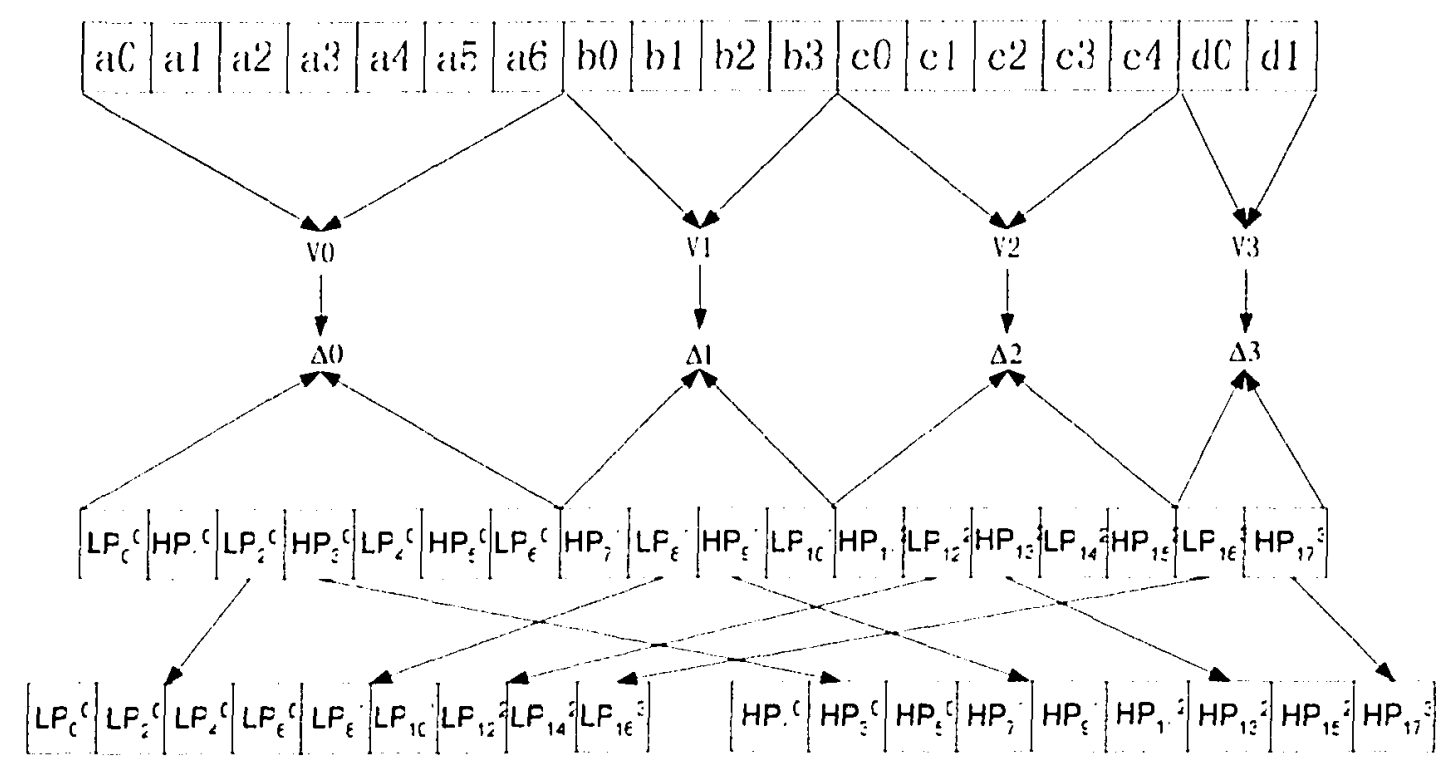

Figure 5.1 Subsampling strategy for multiple regions [25]

First, one dimensional signal of 18 pixels is partitioned into four regions, denoted by $\mathrm{j}, j=0, \ldots, 3$. An individual segment of coefficients is produced for the representation of each region. The wavelet coefficients are denoted by $L P_{i}^{\prime}$ and $H P_{i}^{\prime}$, where $I$ is the label of the region and $i$ is the index of the coefficients. For example, first signal segment starts with even-index, so its lowpass coefficients are chosen at even-position coefficients, $\left\{L P_{0}^{0}, L P_{2}^{0}, L P_{4}^{0}, L P_{\circ}^{0}\right\}$, and highpass filtered representations are selected from oddposition coefficients, $\left\{H P_{1}^{0}, H P_{3}^{0}, H P_{5}^{0}\right\}$. However, second segment starts with odd positions and alternative subsampling method is used for its representation, odd subsampling for lowpass coefficients and even subsampling for highpass coefficients. After subssampling, all lowpass coefficients from multiple regions are placed in corresponding positions in lowpass band and all highpass coefficients from multiple 
regions are placed in corresponding positions in highpass band. Note that the constraints imposed by equation 5.1 are satisfied with the presented multiple regions decomposition strategy.

We can represent partitioned subvolumes (ROIs) of image sequence using the volumetric subsets of wavelet coefficients constructed with the method explained above. These volumetric subsets of wavelet coeflicients consist of an entire transform volume of same size as that of a particular ROI. This property facilitates the use of conventional wavelet coefficient plane coders for multiple, arbitrary-shape volumetric coding.

\subsection{D MRMQ Coding Based on 3D SPIHT Algorithm}

Based on the representation for multiple volumetric ROIs, 3D MRMQ-SPIHT coding algorithm is implemented. First, we describe a coding method derived from 3D SPIHT.

\subsubsection{D MRMQ-SPIHT Algorithm with Sets of Descendant Labels}

As described in previous section, the representations for multiple volumetric regions constitute the conventional wavelet coefficient volume. Thus, the spatio-temporal orientation trees can be constructed by using the conventional SPIHT coding algorithm. However, when coefficients are to be encoded, an important issue is that there exist 
coefficients from different volumetric regions within the same spatio-temporal orientation tree. This problem can be solved by using different labels introduced in [1] [25], which help to distinguish the coefficients from different volumetric regions. For encoding of the transform coefficients pertaining to region 1, we partition all the spatiotemporal orientation trees using the conventional method and encode the information about wavelet coefficients in a particular volumetric region in LIP and LIS sorting stages.

To describe this approach, the notion of sets of descendent labels is defined for multiple regions of image compression in [1] [25] to help reader understand the method. This can be directly extended to 3D case as:

$$
\Psi(\mathbf{i})=\bigcup_{\mathbf{j}=(\mathbf{i})}\{l(\mathbf{j}) \cup \Psi(\mathbf{j})\}
$$

Where $O(\mathbf{i})$ are the direct descendants of coefficient at index $\mathbf{i}$ and $l(\mathbf{j})$ denotes the label of volumetric region which coefficient with index $\mathbf{j}$ belongs to. If $\mathbf{j} \in \Delta_{k}$, then $l(\mathbf{j})=k$. Therefore, $\Psi(\mathbf{i})$ denotes the set of descendent labels of the coefficient with index $\mathbf{i}$. It is obviously that $\Psi(\mathbf{j})=\varnothing$ if coefficient with index $\mathbf{j}$ has no offsprings. In conventional SPIHT algorithm, list of insignificant pixels (LIP) and list of insignificant sets (LIS) are initialized by using the set of wavelet coefficients within the lowest resolution subband, $H$. However, in case volumetric region $k$ is to be encoded, these lists should be initialized by using 


$$
\begin{gathered}
H_{k}^{L . P P}=\left\{\mathbf{j} \mid \mathbf{j} \in\left(H \cap \Delta_{k}\right)\right\} \\
H_{k}^{L I S}=\{\mathbf{j} \mid k \in \Psi(\mathbf{j}), \mathbf{j} \in H\}
\end{gathered}
$$

In other words, LIP is initialized using the coordinates, $\mathbf{j} \in H_{k}^{L / P}$ and LIS is initialized by the root coefficients with coordinates $\mathbf{j} \in H_{k}^{l . l s}$. In the sorting phases, only the spatiotemporal orientation trees having coefficients in volumetric region $k$, i.e., $k \in \Psi(\mathbf{j})$, are partitioned and encoded. For other coefficient trees, we just skip them and no bits are used for encoding them because of their irrelevancy to the volumetric region. During the sorting process, if subtrees having no coefficients in volumetric region $\mathrm{k}$ are encountered, these branches are pruned. After finishing encoding one volumetric region, the algorithm performs the same sorting operation for the next volumetric region until all volumetric regions are processed in this way. This scheme directly extends the original algorithm to encode multiple regions at multiple quality constraints or at a specified quality constraint. Thus, independent bitstreams for each volumetric region are produced and a context based arithmetic coding algorithm can then be used to improve coding efficiency by exploiting the dependencies between significant information of wavelet coefficients.

\subsubsection{D MRMQ-SPIHT Algorithm with Unbalanced Tree}

To further improve the performance of multiple volumetric regions coding, we will adopt unbalanced spatio-temporal orientation tree structure, which is extended from unbalanced 
spatial orientation tree proposed in [1] [25], to construct homogeneous trees where all transformed coefficients in a particular tree belong to the same volumetric region. Recall that in section 4.3 , in case of a single volumetric ROI, the transform coefficients are classified into three categories. By reorganizing the parent-children relations of coefficients in heterogeneous trees, an unbalanced tree structure with coefficients belonging only to the ROIs is created. In case of multiple volumetric regions, we modify the method to construct spatio-temporal orientation tree with the coefficients having same region label. Figure 5.2 illustrates the aforementioned method to construct unbalanced trees for multiple volumetric ROls.

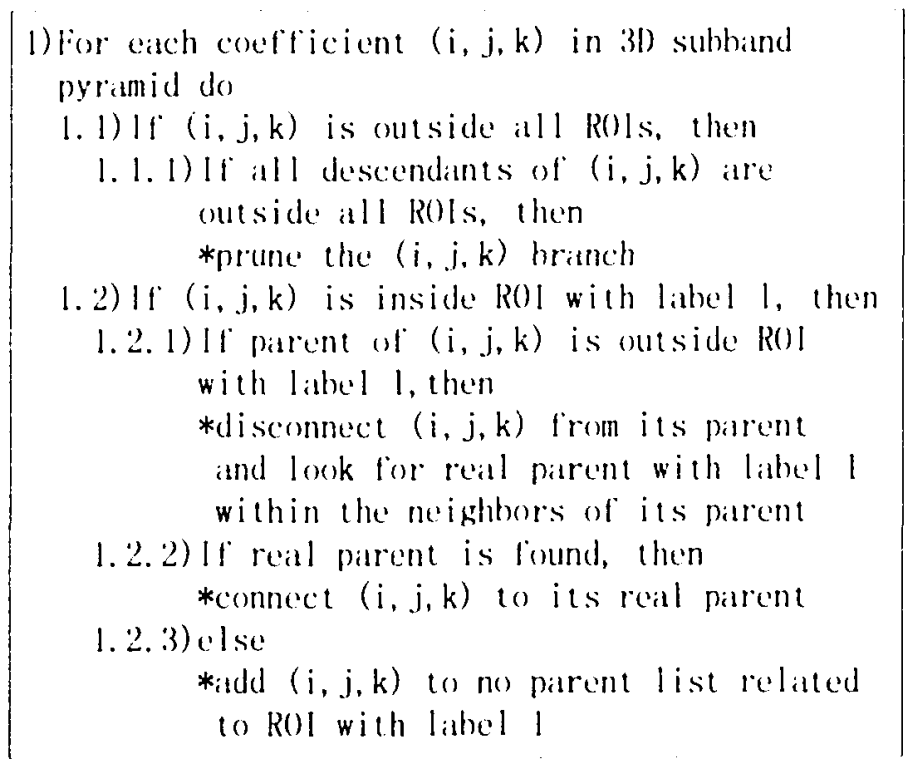

Figure 5.2 Algorithm to construct MRMQ unbalanced trees

When a child coefficient and its parent belong to different volumetric ROIs, the label of child coefficient is used to look for the real parent within the neighbors of its current 
parent. If such a real parent is found, the child coefficient is reconnected to its real parent. After the process of reconnection of coefficients having different label with their parent coefficients to their real parent coefficients, all coefficients in each spatio-temporal coefficient tree will be of the same label.

In coding the coefficients of multiple volumetric ROIs, unbalanced tree structure is applied and the way to access descendents along the coefficient trees is modified. In each round of sorting pass, LIP is initialized with only the coefficients within $H_{k}^{L . t \prime}$ and LIS is initialized by using the root coefficients belonging to $H_{k}^{L J S}$. The way that gets the descendents of a coefficient is along the unbalanced tree, to which the coefficient is belong, instead of normal tree in sorting pass. This method prevents including coefficients that do not belong to the ROIs through a sorting pass as opposed to conventional SPIHT algorithm. The flowchart of 3D MRMQ-SPIHT algorithm based on unbalanced tree structure is illustrated in Figure 5.3. 


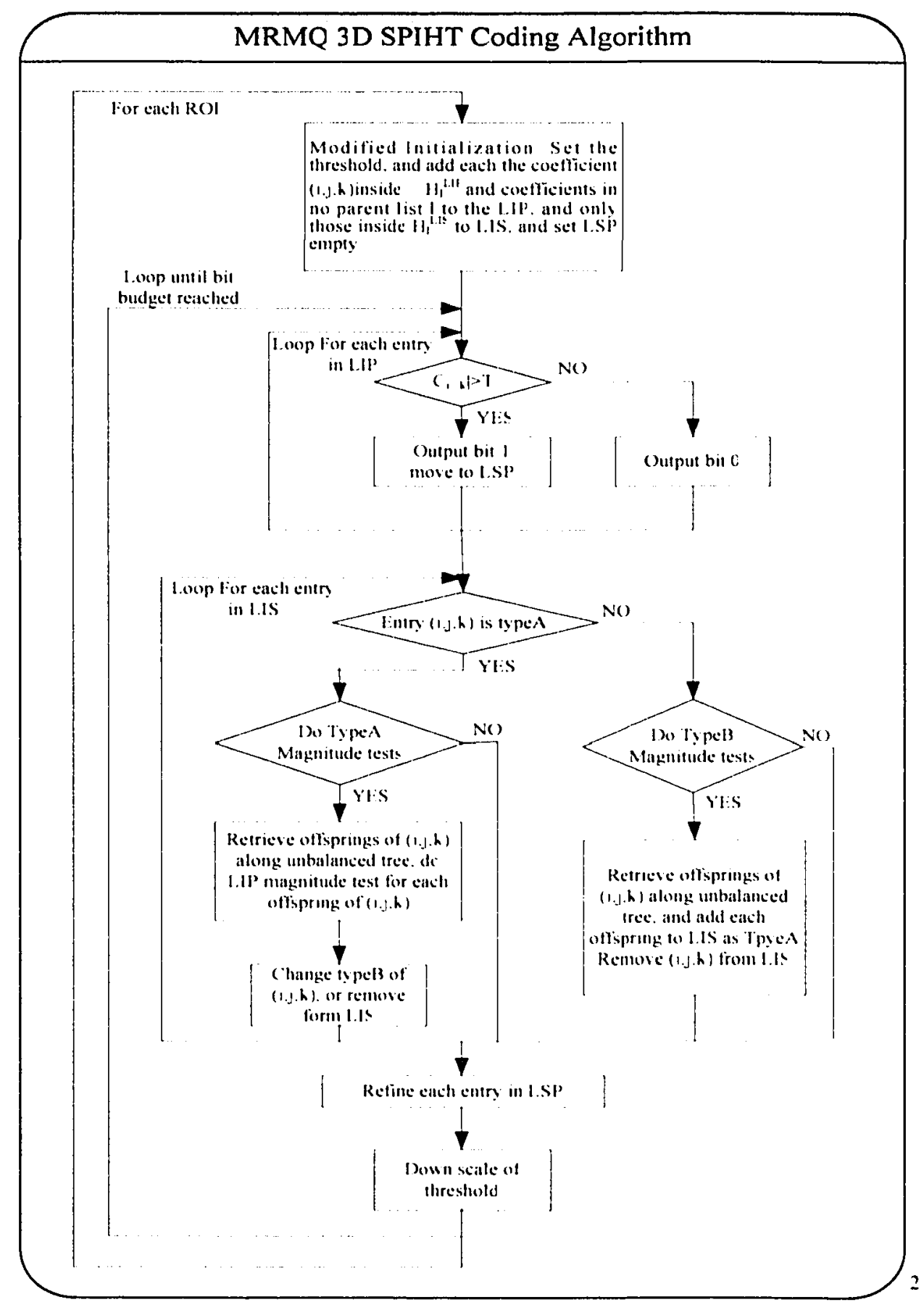

Figure 5.3 Flowchart of 3D MRMQ-SPIHT algorithm

${ }^{2}$ TypeA and TypeB are defined in Chapter 3, on page 34. 


\subsection{Bit Stream Structure for Multiple ROIs}

When the bitstreams for all multiple volumetric ROIs are produced, they should be put together to form a single bitstream for applications of storage and transmission. Note that all substreams are independent each other, so a ROI header is used for indicating the information of each substream. These headers are important for the decoder to reconstruct image sequence correctly. To minimize the overhead of header information, a global header is adopted to hold common information for the image sequence. The bitstream structure with four substreams is shown in Figure 5.4.

In ROI header,

- Mean: Mean of the coefficients within ROI

- Max_coef_bits: Maximal number of bit of the coefficients within ROI

- Num_lotal_bits: Total number of bits used to encode ROI

In Global header,

- Temp_levels: Number of temporal transform levels

- Spatio_levels: Number of spatial transform levels

- Size_frames: The size of frames in the group of frames (GOF)

- Size_rows: The size of rows in each frame

- Size_columns: The size of columns in each frame 
Using this structure, decoder can reconstruct the image sequence with multiple ROIs at the desired qualities.

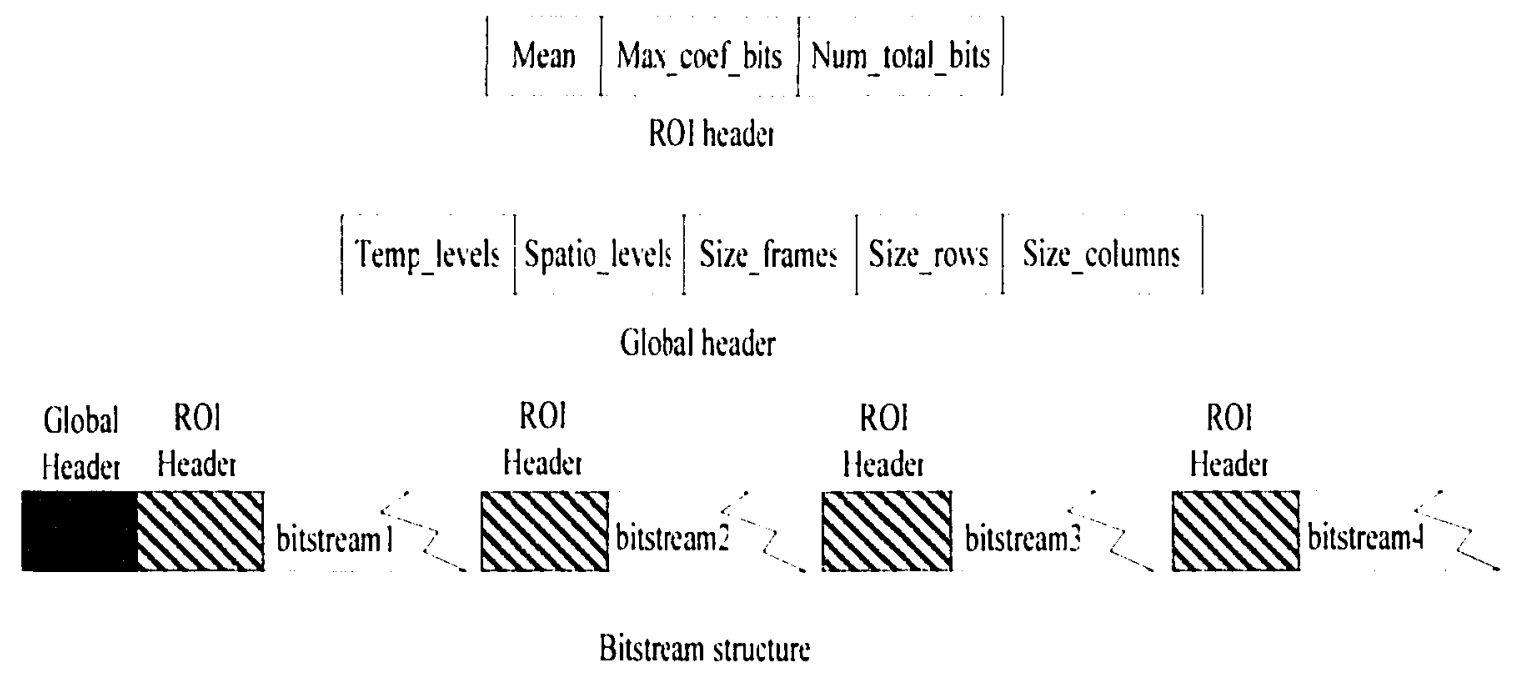

Figure 5.4 Bitstream structure in 3D MRMQ coding

\subsection{Summary}

In this chapter, we discussed multiple coding of 3D ROIs. Wavelet representation for multiple regions is presented and 3D MRMQ-SPIHT algorithm based on unbalanced tree structure is proposed. In the following chapter, we present experimental results obtained by the proposed coding technique and provide a comparison of its R-D performance with state-of-the-art coding algorithms [6]-[8]. 


\section{Chapter 6}

\section{Experimental Results}

In this chapter, we evaluate the rate-distortion performance of the unbalanced tree algorithm (UT-SPIHT) based on 3D SPIHT and perform comparisons with other methods including dyadic-wavelet SPIHT (DY-SPIHT), packet-wavelet SPIHT (PK-SPIHT) and optimal tree SPIHT algorithms (OT-SPIHT) for two cases: i) compression of MR volumetric datasets and ii) compression of video data.

\subsection{Selection of Wavelet Basis}

For wavelet basis used in our experiments, some constraints should be taken into consideration. As discussed in chapter 2, smoothness, orthonormality and linear phase properties are desired when choosing a wavelet basis for compression of images. In practice, orthonormality and linear phase properties are not compatible, i.e. a wavelet can not have both of these properties simultaneously. In general, biorthogonal wavelet basis are selected for decomposition of images because they are approximately unitary and of linear phase. By combining biorthogonal wavelet basis and SA-DWT, the distortion for 
each ROI resulting from the quantization of coefficients in the transform domain can be used in place of the distortion calculated in the image domain [25], that is

$$
D^{\left(w_{1}^{\prime}\right)} \approx D^{\left(\lambda_{1}\right)}
$$

Where $D^{\left(v_{1}\right)}$ and $D^{\left(a_{1}\right)}$ are the distortions of region i measured in image domain and transform domain respectively. Thus, we can control the distortion without introducing additional computational cost in the transform domain using odd length symmetric biorthogonal wavelet basis for image sequences with multiple ROls.

The well-known Daubechies $9 / 7$ and $5 / 3$ biorthogonal wavelets are used in our experiments due to their excellent performance in image coding [13] [33]. Furthermore, these wavelets also satisfy the desired properties mentioned above.

\subsection{Compression of Volumetric Medical Datasets}

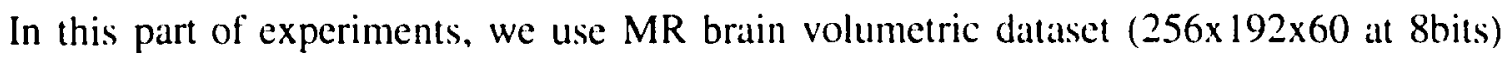
obtained from the Ottawa General Hospital. Some of the slices from the original sequence are shown in Figure 6.1. In order to perform wavelet decomposition, the last slice of the MRI sequence is duplicated four times and copied slices are padded at the end of the sequence to make the number of whole sequence power of 2, (i.e. 64). A ROI mask is created by generating a contour of the part of the brain. Figure 6.2 illustrates the ROI 
masks for slice number 10 and slice number 20 of the image sequence. For all experiments in this thesis, rate is computed from actual (compressed) file sizes.
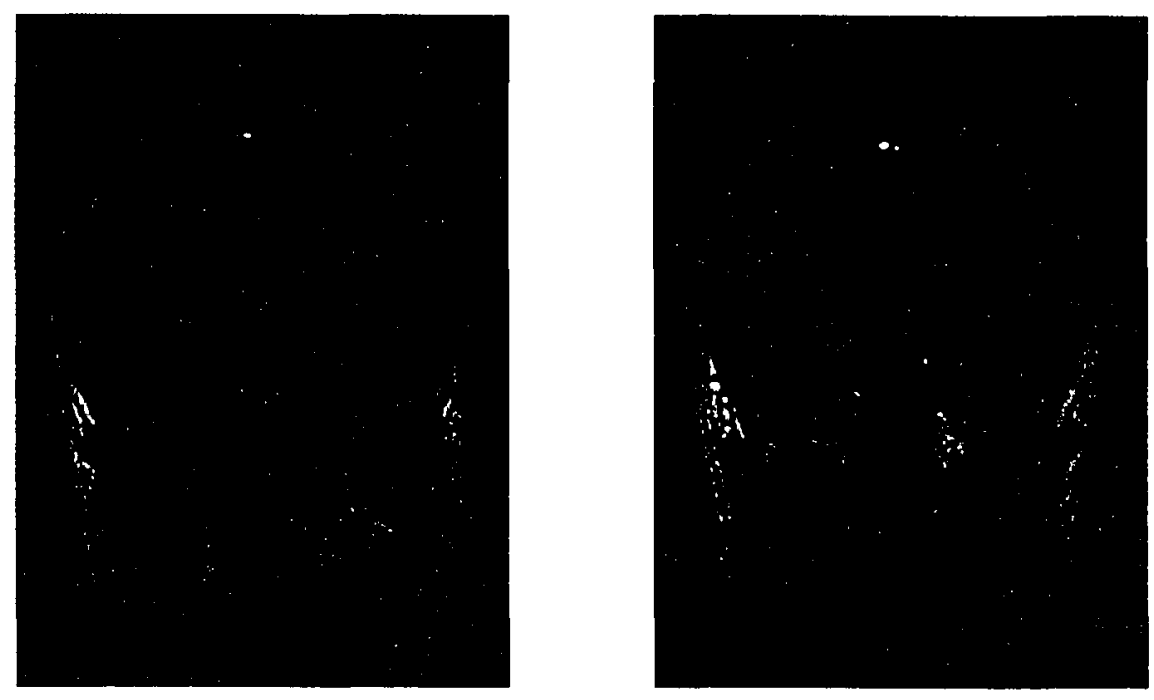

Figure 6.1 Original slice 10 (left) and slice 20 (right) of image sequence
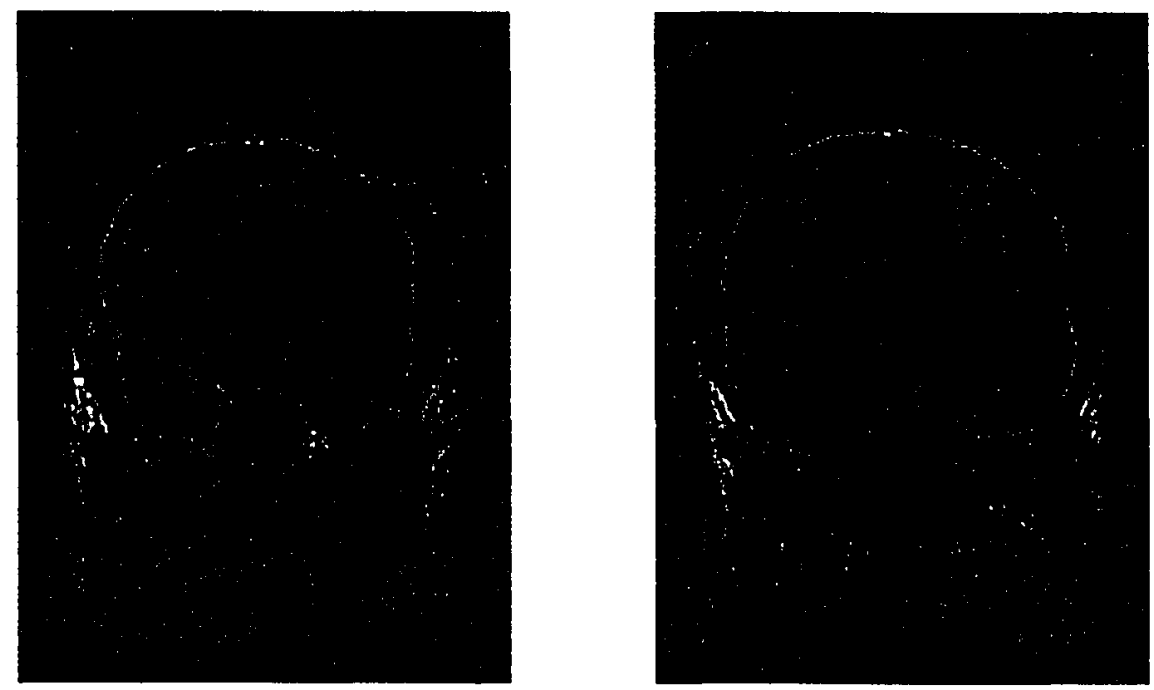

Figure 6.2 Pre-segmented slice number 10 (left) and slice number 20 (right) of image sequence 
The experiment is first performed with different interslice transform levels and fixing the GOS size at 64 . In Table 6.1 and table 6.2, the PSNR results by biorthogonal $9 / 7$ and biorthogonal $5 / 3$ at rate $0.1 \mathrm{bpp}$ and $0.4 \mathrm{bpp}$ are compared.

\begin{tabular}{|c|c|c|}
\hline $\begin{array}{l}\text { Interslice } \\
\text { level }\end{array}$ & $\begin{array}{l}\text { Average ROI PSNR (dB) using } \\
9 / 7 \text { wavelet transform }\end{array}$ & $\begin{array}{l}\text { Average ROI PSNR (dB) using } \\
5 / 3 \text { wavelet transform }\end{array}$ \\
\hline 2 & 36.39 & 35.93 \\
\hline 3 & 36.24 & 35.70 \\
\hline 4 & 36.11 & 35.47 \\
\hline 5 & 36.04 & 35.33 \\
\hline
\end{tabular}

Table 6.1 Average ROI PSNR (dB) at rate 0.1 bpp with different interslice transform levels (GOS size is 64)

\begin{tabular}{|c|c|c|}
\hline $\begin{array}{l}\text { Interslice } \\
\text { level }\end{array}$ & $\begin{array}{l}\text { Average ROI PSNR (dB) using } \\
9 / 7 \text { wavelet transform }\end{array}$ & $\begin{array}{l}\text { Average ROI PSNR (dB) using } \\
5 / 3 \text { wavelet transform }\end{array}$ \\
\hline 2 & 43.65 & 43.21 \\
\hline 3 & 43.47 & 42.89 \\
\hline 4 & 4.3 .36 & 42.72 \\
\hline 5 & 43.29 & 42.60 \\
\hline
\end{tabular}

Table 6.2 Average ROI PSNR (dB) at rate 0.4 bpp with different interslice transform levels (GOS size is 64)

The results show that $9 / 7$ wavelet transform has better $R-D$ performance than $5 / 3$ wavelet. However, increasing the number of interslice decomposition levels has little effect on compression performance.

Moreover, we investigated the effects of different GOS sizes on compression performance. Again $9 / 7$ and $5 / 3$ wavelets are used to perform the experiments. The number of spatial decomposition levels is chosen as 5 and interslice decomposition level 
is chosen as 2 . Here, to form a $3 \mathrm{D}$ dataset, the whole image sequence is divided into groups of 8, 16, 32 and 64 slices to form different GOS sizes. ROI PSNRs are averaged over 64 slices. Table 6.3 and Table 6.4 provide the PSNR results for different GOS sizes at rates of $0.1 \mathrm{bpp}$ and $0.4 \mathrm{bpp}$, respectively.

\begin{tabular}{|c|c|c|}
\hline $\begin{array}{c}\text { G(S } \\
\text { size }\end{array}$ & $\begin{array}{c}\text { Average } \mathrm{ROI} \text { PSNR (dB) using } \\
9 / 7 \text { wavelet transform }\end{array}$ & $\begin{array}{l}\text { Average R(I PSNR (dB) using } \\
5 / 3 \text { wavelet transform }\end{array}$ \\
\hline 8 & 36.01 & 35.53 \\
\hline 16 & 36.20 & 35.73 \\
\hline 32 & 36.32 & 35.88 \\
\hline 64 & 36.39 & 35.93 \\
\hline
\end{tabular}

Table 6.3 Average ROI PSNR $(\mathrm{dB})$ at $0.1 \mathrm{bpp}$ for different GOS sizes

\begin{tabular}{|c|c|c|}
\hline $\begin{array}{c}\text { GOS } \\
\text { size }\end{array}$ & $\begin{array}{l}\text { Average R()I PSNR (dB) using } \\
9 / 7 \text { wavelet transform }\end{array}$ & $\begin{array}{l}\text { Average R( I PSNR (dB) using } \\
5 / 3 \text { wavelet transform }\end{array}$ \\
\hline 8 & 43.24 & 42.95 \\
\hline 16 & 43.47 & 4.3 .14 \\
\hline 32 & 43.59 & 4.3 .11 \\
\hline 64 & 4.3 .65 & 43.21 \\
\hline
\end{tabular}

Table 6.4 Average ROI PSNR (dB) at 0.4hpp for different GOS sizes

The results in Table 6.3 and Table 6.4 show that average PSNR of the ROI increase with larger GOS size. However, one must note that increasing GOS size increases the memory requirements of the algorithm. If we assume that 8 bytes are required to store the wavelet coefficients, an 8 -slice $256 \times 192$ pixel GOS requires about 3MB of memory, 
while a 64 -slice GOS requires $24 \mathrm{MBs}$. Once again, the R-D performance using $9 / 7$ wavelet is slightly better than that of $5 / 3$ wavelet for different GOS sizes.

To compare the proposed UT-SPIHT coding algorithm with DY-SPIHT, PK-SPIHT, and OT-SPIHT algorithms, we perform experiments by setting the number of decomposition levels in the spatial domain to 5 except for DY-SPIHT, in which spatial transform levels are equal to interslice transform levels. The number of decomposition levels between the slices is set to 2, 3,4, and 5 according to the GOS size of the image sequence, i.e. 8, 16, 32, and 64 respectively. Results of average PSNRs for the ROI at bit rate of $0.4 \mathrm{bpp}$ are given in Table 6.5 .

\begin{tabular}{|c|c|c|c|c|}
\hline GOS size & UT-SPIHT & OT-SPIHT & PK-SPIHT & DY-SPIHT \\
\hline 8 & 4.3 .24 & 42.46 & 41.27 & 41.34 \\
\hline 16 & 4.3 .25 & 42.39 & 41.99 & 42.20 \\
\hline 32 & 43.21 & +2.46 & 41.93 & 42.26 \\
\hline 64 & 43.29 & +2.38 & 41.97 & 42.30 \\
\hline
\end{tabular}

Table 6.5 Comparison of average ROIs' PSNR (dB) with different 3D coding methods at $0.4 \mathrm{bpp}$

As can be seen from Table 6.5, the proposed UT-SPIHT algorithm performs better than the other three algorithms for different GOS sizes. For GOS size of 16, it performs on average $0.78 \mathrm{~dB}$ better than the OT-SPIHT algorithm. Figures 6.3 and 6.4 show the RD performance of the proposed algorithm at average rates of 0.1 bpp and $0.4 \mathrm{bpp}$. respectively for GOS size of 64 using $9 / 7$ wavelet with 5 transform levels for all 
dimensions. For reference, results obtained by the other three algorithms are also included.

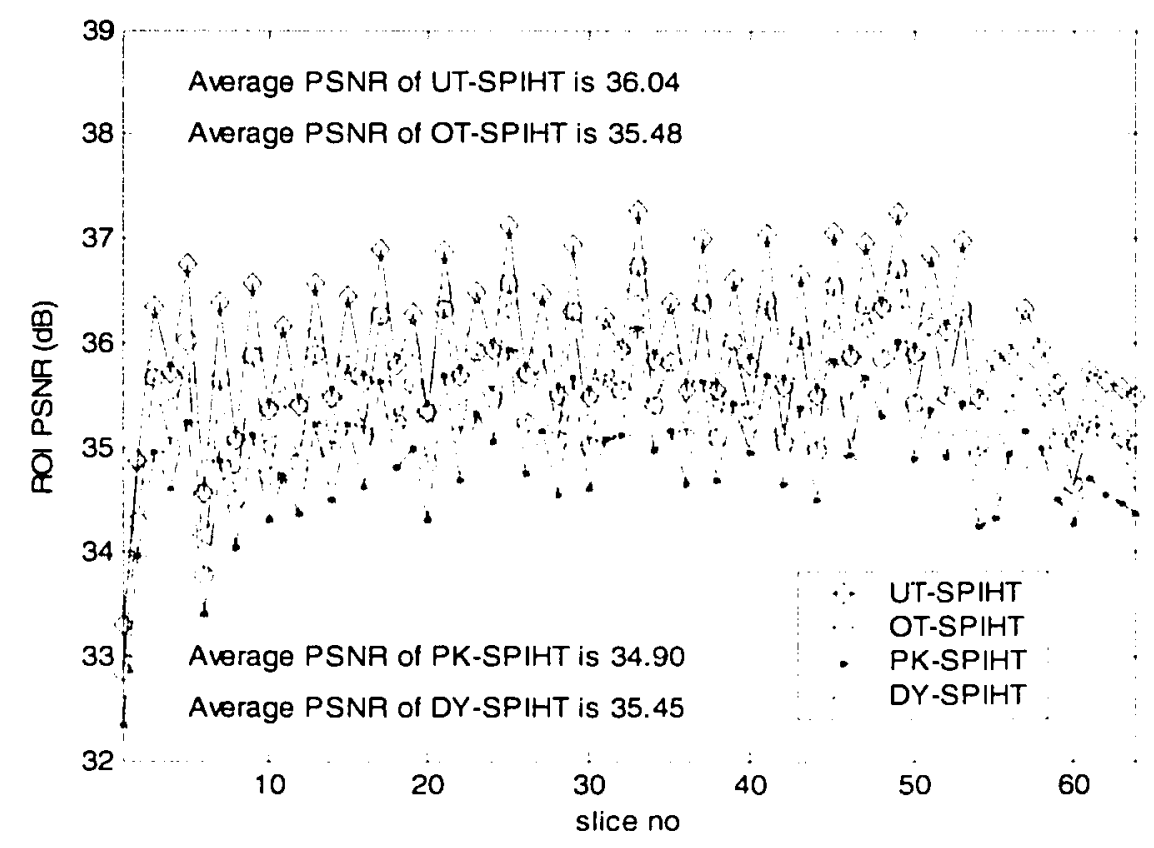

Figure 6.3 Comparison of ROI PSNRs at rate $0.1 \mathrm{bpp}$ 


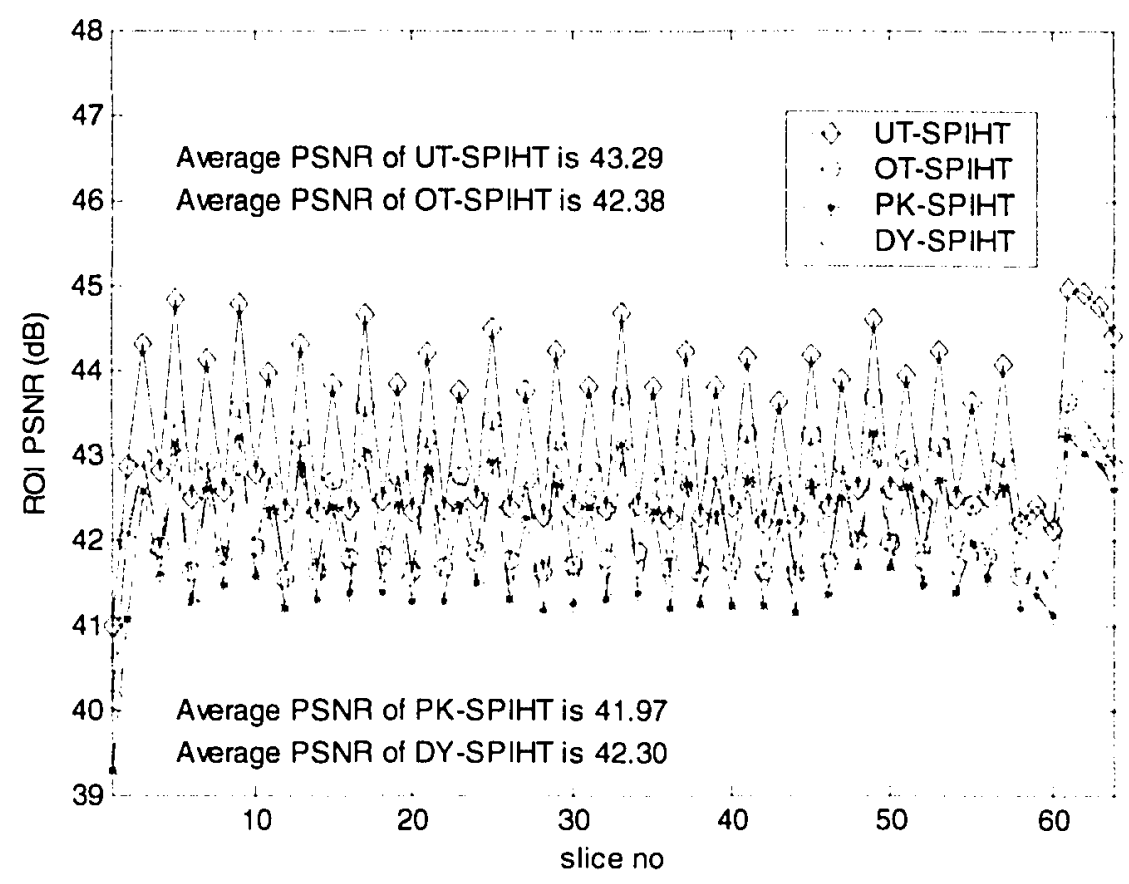

Figure 6.4 Comparison of ROI PSNRs at rate $0.4 \mathrm{bpp}$

The two figures show that the ROI PSNR of each slice using UT-SPIHT algorithm is better than that of other algorithms at both bit rates. The improvement is up to $1.14 \mathrm{~dB}$ at rate $0.1 \mathrm{bpp}$ and up to $1.32 \mathrm{~dB}$ at rate $0.4 \mathrm{bpp}$. The average ROI PSNRs for the four algorithms using the same settings in Figure 6.3 and 6.4 at rates changing from 0.1 to 1.0 bpp are shown in Figure 6.5 . 


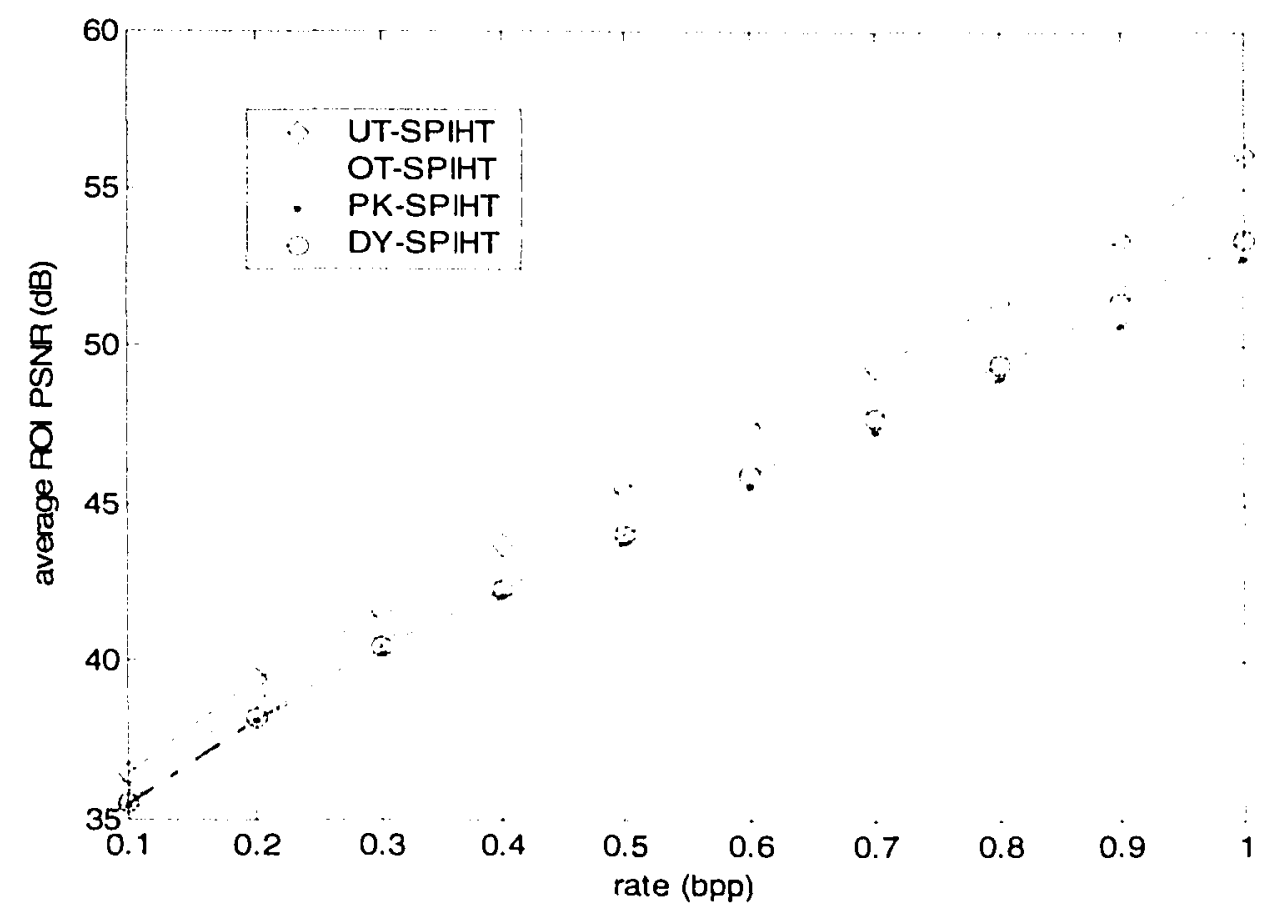

Figure 6.5 Average ROIs' PSNR of different algorithms at rate from 0.1 to $1.0 \mathrm{bpp}$

On average, UT-SPIHT algorithm outperforms the OT-SPIHT algorithm by $0.5 \mathrm{~dB}$, DY-SPIHT algorithm by 1.0 , and PK-SPIHT algorithm by $1.1 \mathrm{~dB}$. The reconstructed image slice 32 using UT-SPIHT with GOS of 64 and 16 at bit rate 0.1 and 0.5 bpp are shown in Figure 6.6 and 6.7. Note that slice 32 is at the boundary of GOS in one coding unit. 

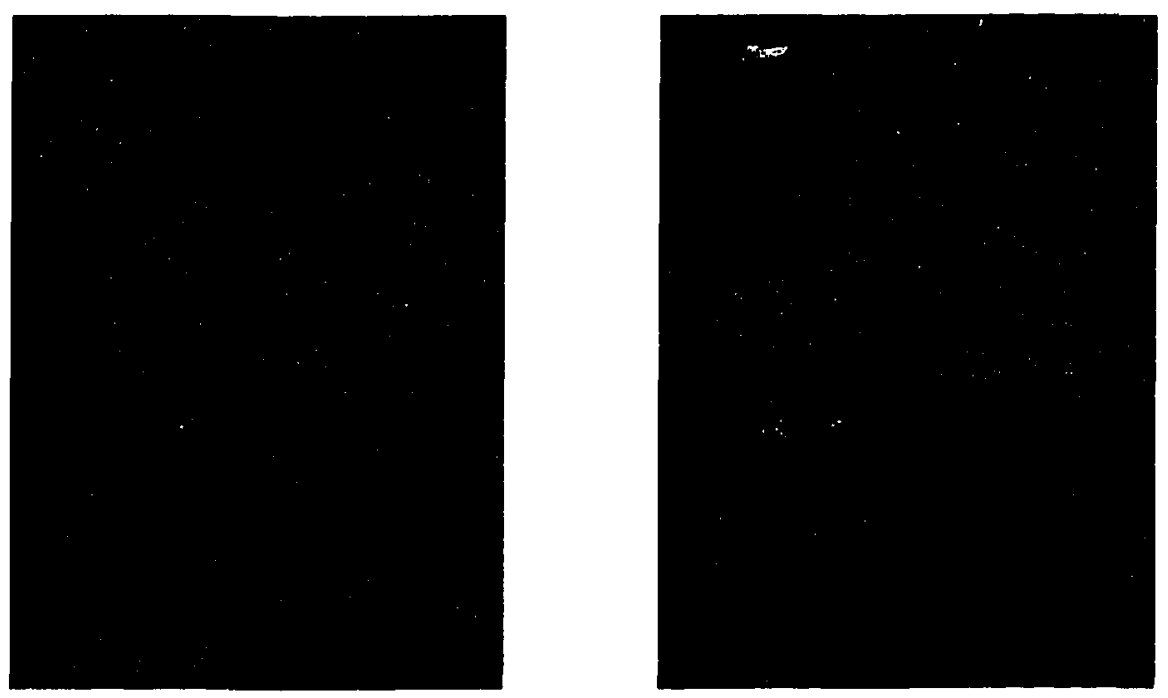

Figure 6.6 Reconstructed slice 32 at rate $0.1 \mathrm{bpp}$ (left, ROI PSNR is $36.03 \mathrm{~dB}$ ) and $0.5 \mathrm{bpp}$ (right, ROI PSNR is $44.06 \mathrm{~dB}$ ) with GOF $=64$ using UT-SPIHT
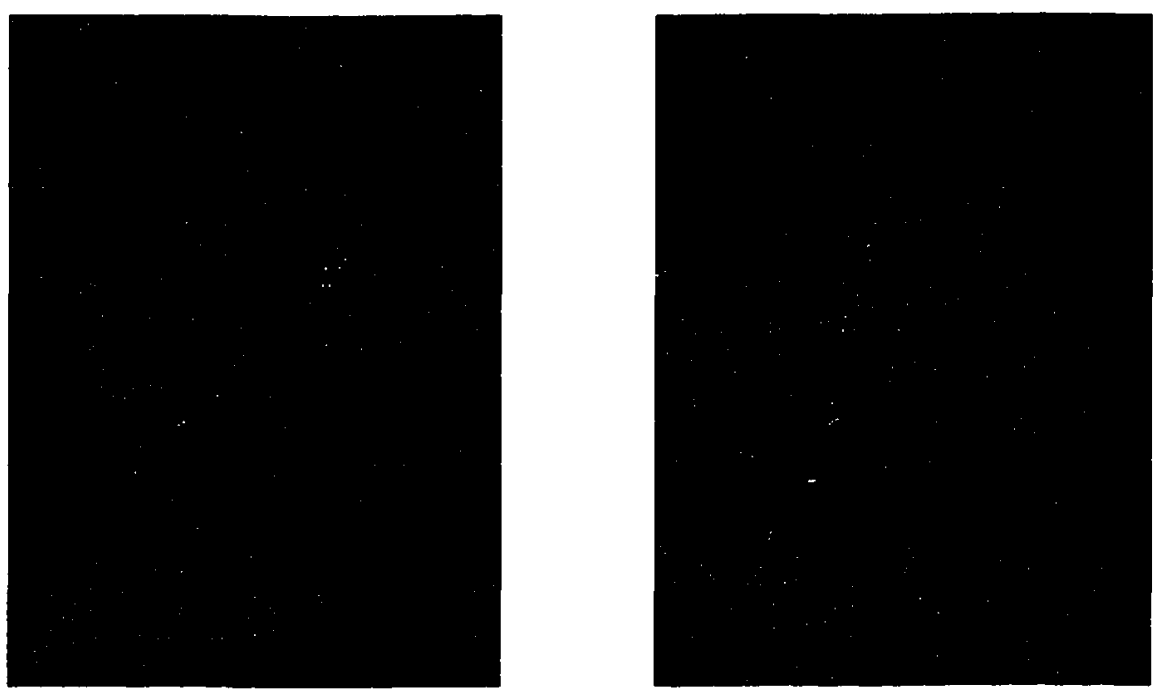

Figure 6.7 Reconstructed slice 32 at rate $0.1 \mathrm{bpp}$ (left, ROI PSNR is $34.06 \mathrm{~dB}$ ) and $0.5 \mathrm{bpp}$ (right, ROI PSNR is $41.76 \mathrm{~dB}$ ) with GOF $=16$ using UT-SPIHT 
The $128 \times 96$ rectangular regions, which have the left corner at position $(40,45)$, of slices in Figure 6.6 and Figure 6.7 are zoomed and shown in Figure 6.8 and Figure 6.9.
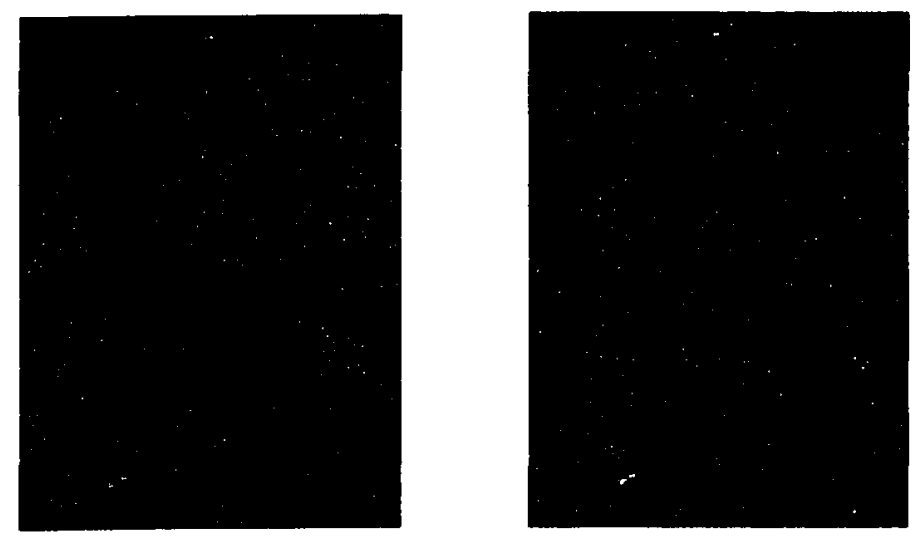

Figure 6.8 Zoomed rectangle of reconstructed slice 32 at rate $0.1 \mathrm{bpp}$ (left) and $0.5 \mathrm{hpp}$ (right) with GOF=64 using UT-SPIHT
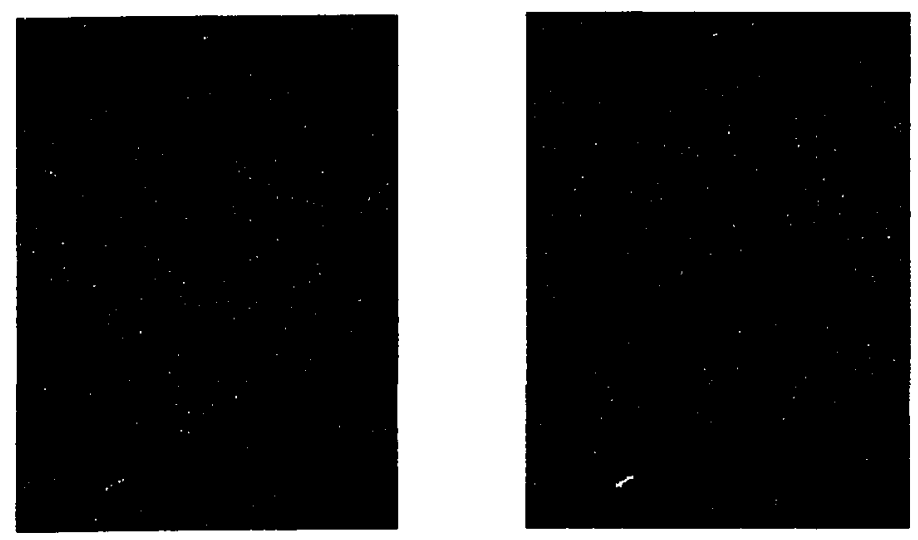

Figure 6.9 Zoomed rectangle of reconstructed slice 32 at rate $0.1 \mathrm{bpp}$ (left) and $0.5 \mathrm{bpp}$ (right) with GOF=16 using UT-SPIHT 


\subsection{MRMQ Coding Performance}

A significant feature of the unbalanced tree structure is that it can be used to implement a multiple-region multiple-quality coding algorithm (MRMQ-SPIHT) to support compression of multiple ROIs at different qualities. Since other 3D coding algorithms do not support encoding of multiple ROIs, we compare the R-D performance of the MRMQSPIHT algorithm with conventional dyadic 3D SPIHT in this section. First, we consider that the image sequence is partitioned into two parts, the region that encloses the brain and the background. Conventional 3D SPIHT is used to compress the entire image sequence and MRMQ-SPIHT algorithm is used to compress the ROI and background at different qualities.

In this experiment, $90 \%$ of the bit budget is used to code the ROI and the remaining $10 \%$ is used for coding the background. The GOS size is 64 and the spatial and interslice decomposition levels is chosen as 5 and 2. The same wavelet basis, 9/7, is used in all directions. Figure 6.8 shows the R-D performance of the two coding algorithms. 


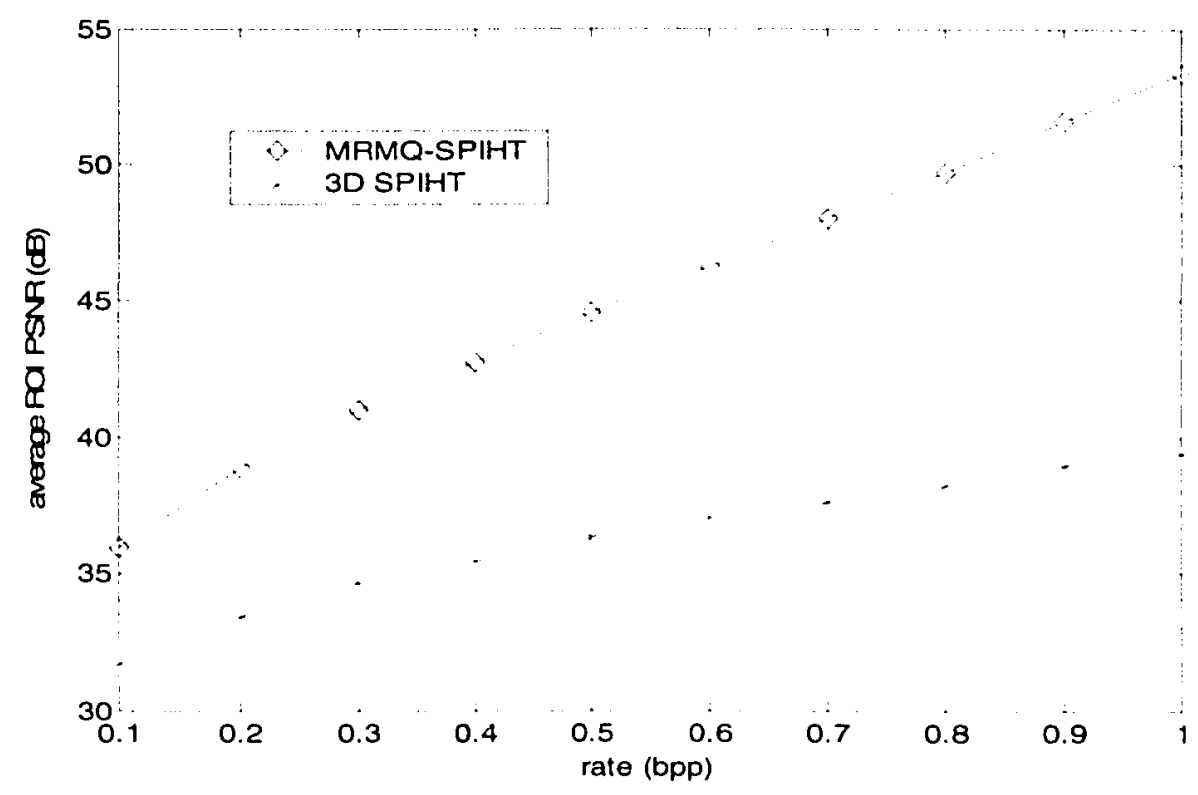

Figure 6.10 Comparison of ROI PSNR

From the Figure 6.8, as expected the ROI PSNR of MRMQ-SPIHT algorithm is better than that of conventional 3D SPIHT algorithm and it quickly becomes nearly lossless after 0.8bpp. Of course, the background PSNR of the proposed algorithm is lower than that of conventional 3D SPIHT, e.g. 30.21 dB with MRMQ and $39.62 \mathrm{~dB}$ with conventional 3D SPIHT at rate 1.0bpp. Assuming that diagnostically significant information is included within the ROI, degradation in the remaining part of the image slices can be tolerated.

Figure 6.9 shows the reconstructed image slices using MRMQ-SPIHT algorithm by bit allocation among the ROI and the background at a total bit rate of $0.2 \mathrm{bpp}$. For 
comparison, reconstructed image slices by conventional 3D SPIHT at the same bit rate are also shown in Figure 6.10.
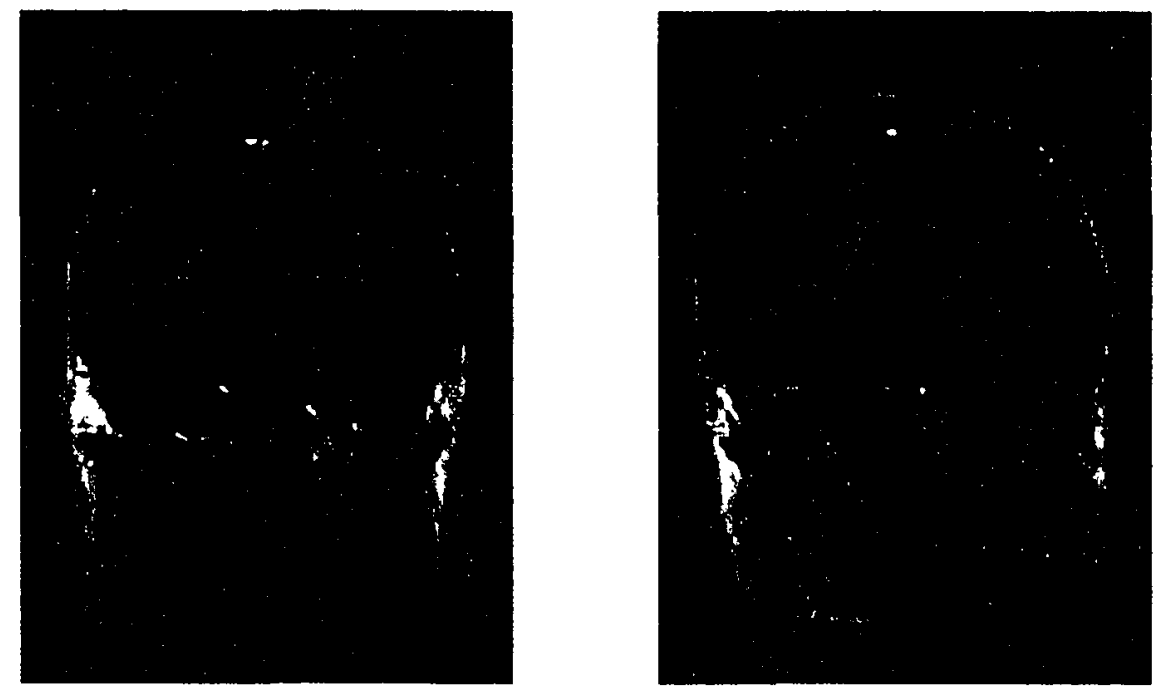

Figure 6.11 Reconstructed image slices using MRMQ-SPIHT algorithm by bit allocation among ROI and background at rate 0.2 bpp
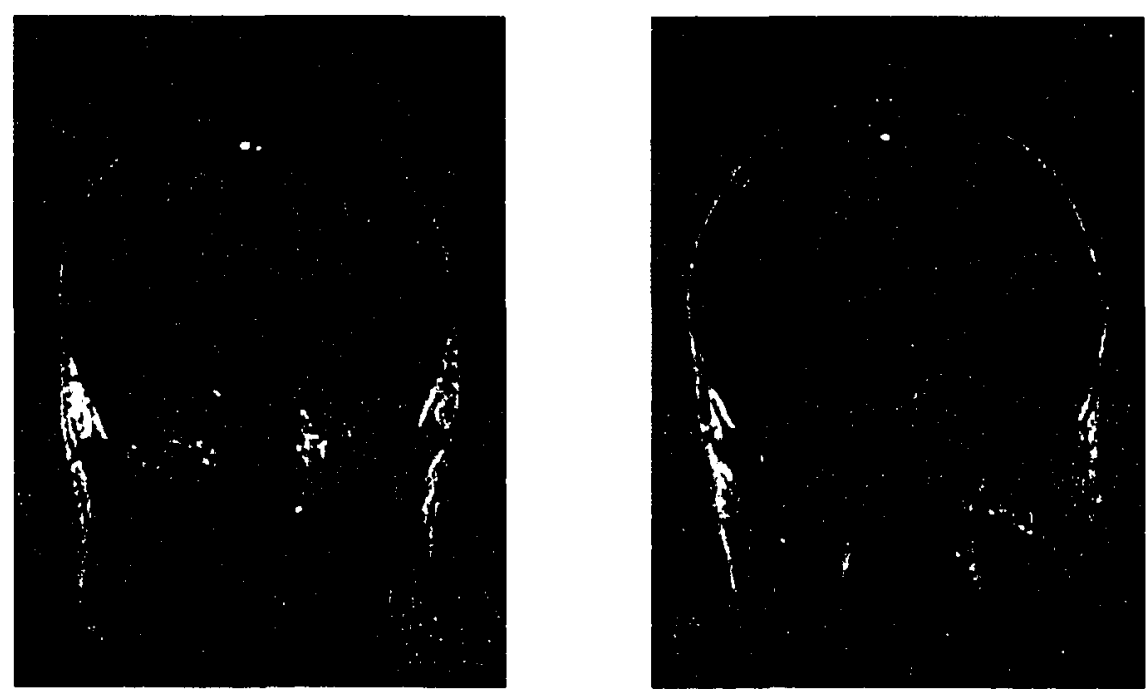

Figure 6.12 Reconstructed image slices by conventional 3D SPIHT at rate $0.2 \mathrm{bpp}$ 
Compared to the UT-SPIHT method presented in section 6.2, we obtain a background at a certain quality instead of a black background, which may be more preferable depending on the application. The advantage of MRMQ coding is that it supports multiple ROIs coding at different rates.

Comparing Figure 6.9 with Figure 6.10, the ROI quality of reconstructed image slices by our proposed algorithm is much better than that using conventional SPIHT, while the background is degraded as only $10 \%$ of the total bit rate has been allocated for encoding the coefficients belonging to the background. At rate $0.2 \mathrm{bpp}$, the average ROI PSNR is $38.88 \mathrm{~dB}$ with compression ratio of $40: 1$ using the MRMQ-SPIHT algorithm. If we want to achieve similar PSNR in ROI using conventional SPIHT, we have to encode this image sequence with rate $0.9 \mathrm{bpp}$. This corresponds to compression ratio of about 9:1. The compressed data file using MRMQ method at rate $0.2 \mathrm{bpp}$ is 4.5 times smaller than that of conventional SPIHT.

\subsection{Compression of Video Sequences}

From the experimental results given in the previous sections, the proposed ROI-based SPIHT coding algorithm based on unbalanced tree is shown to be effective in compression of volumetric datasets. In this section, we evaluate the performance of the proposed algorithm for the compression of video data. Although, conventional video compression using motion compensation provides high compression ratios, such coding 
algorithms have drawbacks due to the following. Motion compensation involves a computationally intensive searching process and it is applied at a fixed resolution which is not easily scalable [6]. 3D wavelet transform applied along spatial and temporal directions has a much lower computation complexity than motion compensation because of no searching operation involved. Using 3D wavelet coding scheme without motion compensation is shown to achieve better coding performance than MPEG-2 [6] [9]. Also, it is shown in [4] that 3D zerotree based methods are comparable with H.263 in terms of R-D performance with the added benefits of scalability and computational simplicity.

The test video is QCIF “Akiyo" sequence which contains 300 frames with each frame at $176 \times 144$ pixels. The frame rate of "Akiyo" video is 30fps. Figure 6.9 shows the example frames of pre-segmented Akiyo sequence. The wavelet used in all the experiments in this section is $9 / 7$ wavelet.
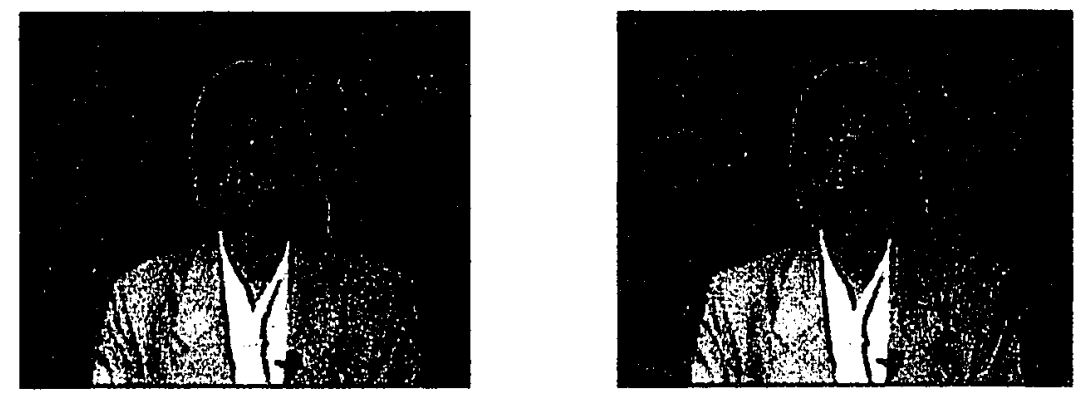

Figure 6.13 Pre-segmented frame 10 and frame 20 of "Akiyo"

In our experiments, without loss of reliability of result and making experiment simple, we use first consecutive 64 frames to do our experiments. First 64 frames are grouped 
together as a GOF for performing 3D wavelet transform. First, to compare UT-SPIHT with other algorithms, an experiment is performed with GOF of 64 and 3 transform levels along all dimensions. Figure 6.12 shows the compression results using different 3D compression methods at bit rate $0.5 \mathrm{bpp}$ or $380.16 \mathrm{~kb} / \mathrm{s}$. The performance of UT-SPIHT is the best among compared algorithms, and it achieves $0.46 \mathrm{~dB}, 1.98 \mathrm{~dB}$ and $5.7 \mathrm{~dB}$ improvement over OT-SPIHT, PK-SPIHT and DY-SPIHT respectively on average over 64 frames.

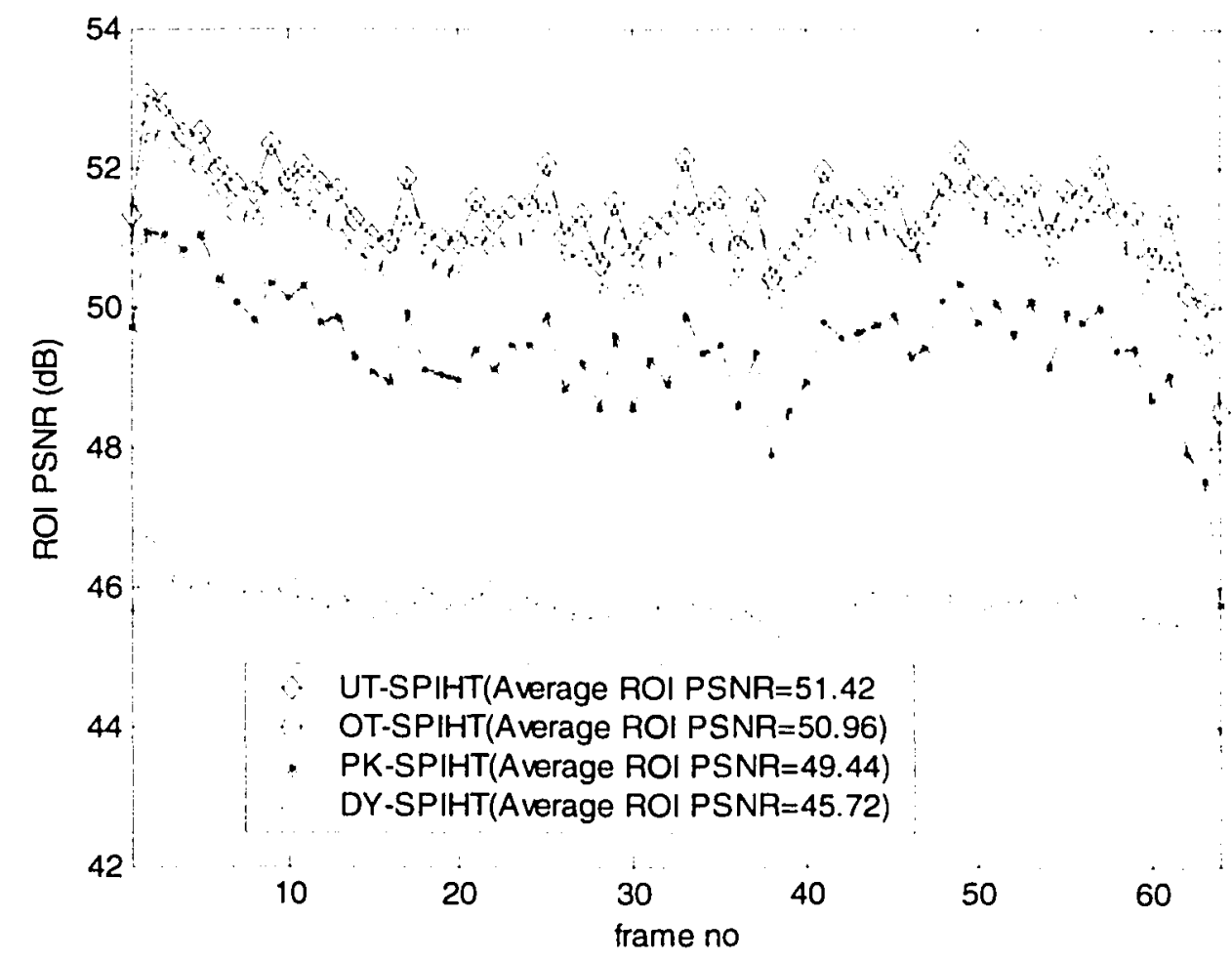

Figure 6.14 Results by different 3D compression methods at bit rate 0.5 bpp or $380.16 \mathrm{~kb} / \mathrm{s}$. 
Figure 6.13 shows the average ROI PSNR curves using different 3D SPIHT algorithms at rates from $76.032 \mathrm{~kb} / \mathrm{s}$ to $760.32 \mathrm{~kb} / \mathrm{s}$. As can be seen the two algorithms UT-SPIHT and OT-SPIHT perform better than the PK-SPIHT and DY-SPIHT algorithms with UT-SPIHT performing slightly better than OT-SPIHT. From the results in the figure, UT-SPIHT produces a PSNR of $51.42 \mathrm{~dB}$ at $380 \mathrm{~kb} / \mathrm{s}$ or $0.5 \mathrm{bpp}$, whereas conventional 3D SPIHT using dyadic 3D transform has a PSNR of $45.72 \mathrm{~dB}$. On average, it achieves $49.36 \mathrm{~dB}$ at $0.4 \mathrm{bpp}$ or $304.128 \mathrm{~kb} / \mathrm{s}$ and achieves compression ratio of $20: 1$. To obtain the same quality, the dyadic SPIHT should encode the sequence at about $0.7 \mathrm{bpp}$ or $530 \mathrm{kbps}$, at which compression ratio is about 11:1.

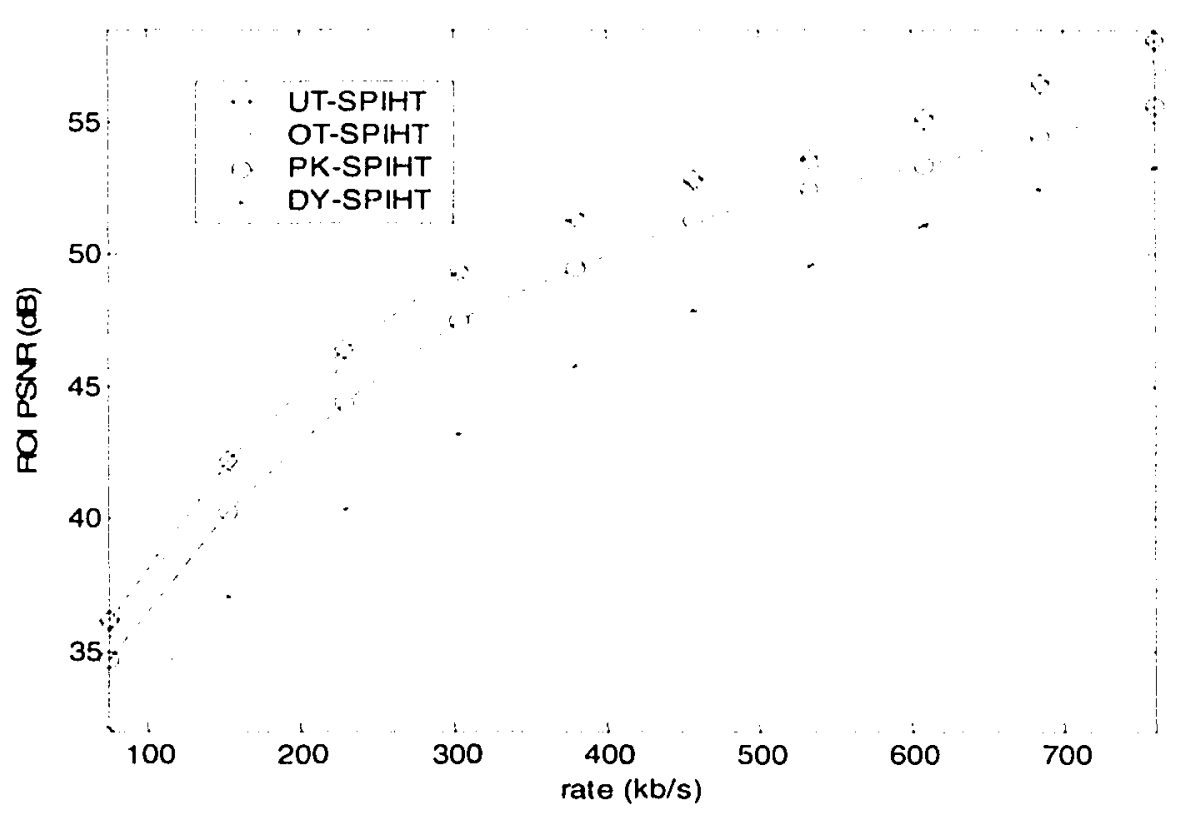

Figure 6.15 Comparison of performance with different 3D coding methods 
Next, we evaluate the performance of UT-SPIHT with different GOF sizes $(64,32$, and 16) for 'Akiyo' video sequence as shown in Figure 6.14. From the figure, increasing the GOF size improves the ROI PSNR slightly, but the improvement is not noticable after the GOF size is increased beyond 32. One must note that larger GOF sizes require more memory and can result in coding delays [6] [9].

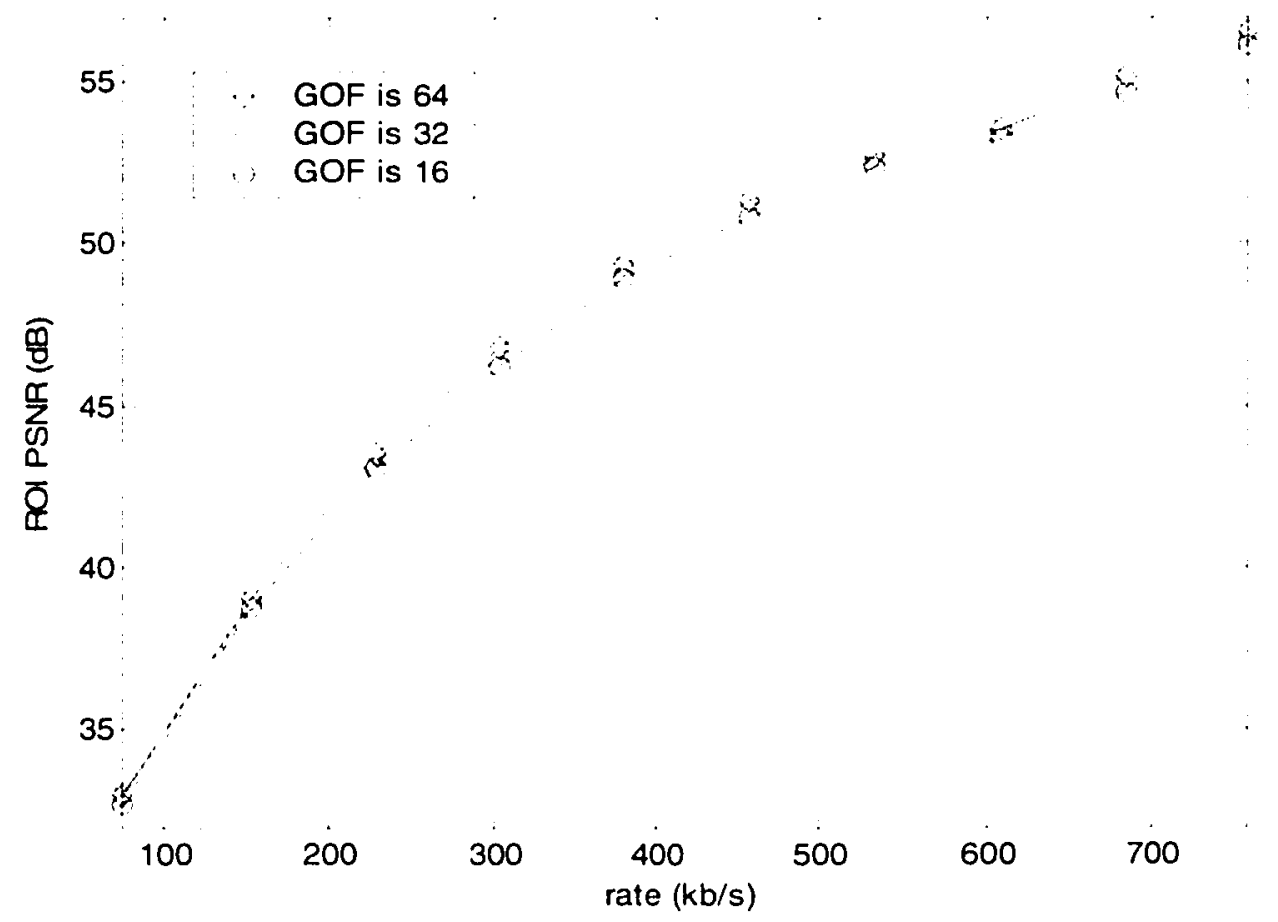

Figure 6.16 Performance of different GOF sizes for 'Akiyo" using UT-SPIHT algorithm

We also performed experiments to compare the performance of UT-SPIHT algorithm with different temporal transform levels. The experiments are carried out with GOF=16, 32 and 64 and 3 levels of spatial transform. The R-D performances for different temporal levels are shown in Figure 6.15, 6.16 and 6.17. We can see that increasing temporal 
transform levels can improve the coding performance. The reason is that more energy compaction in lower subbands is achieved with more levels of temporal transform and UT-SPIHT coding algorithm takes advantage of such energy compaction to achieve better performance.

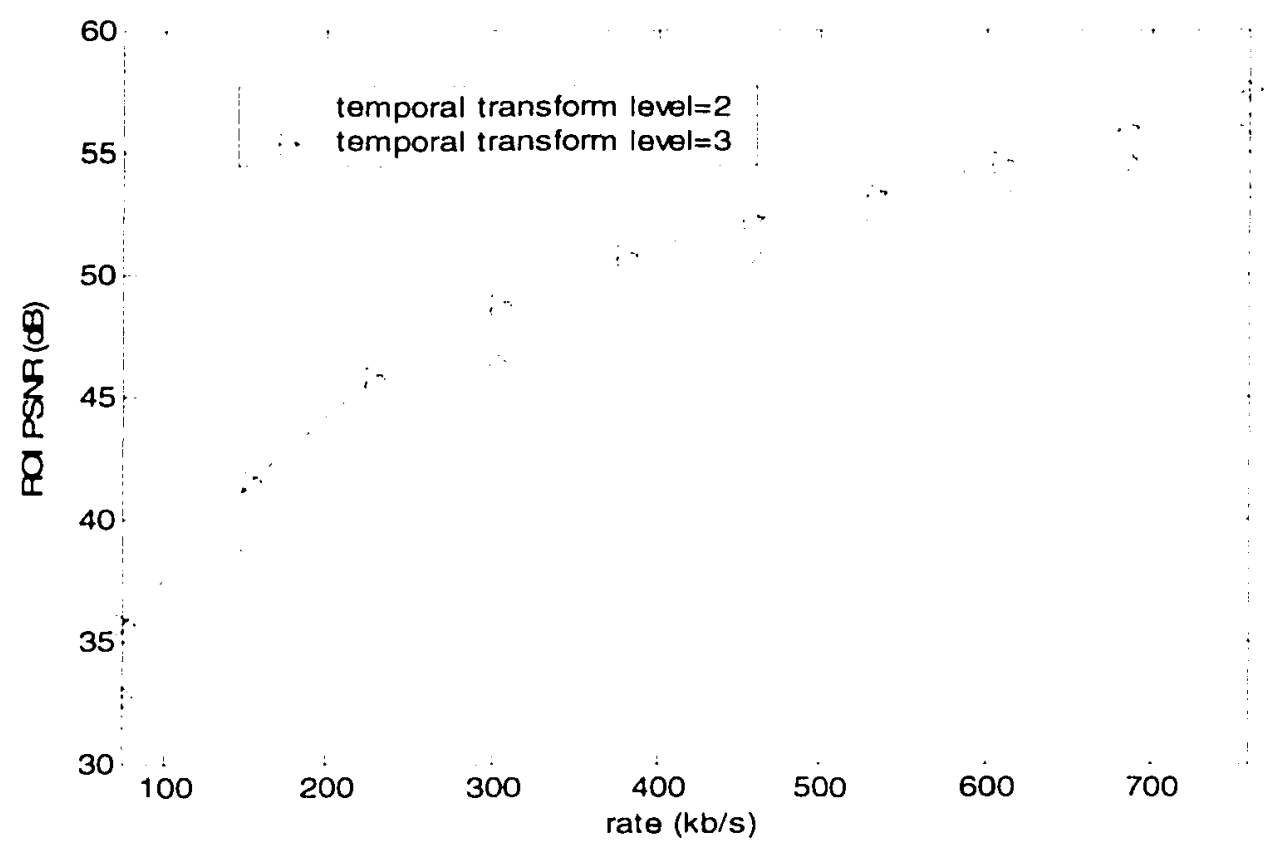

Figure 6.17 Comparison of performance for 'Akiyo' at different temporal transform levels $(\mathrm{GOF}=16)$ 


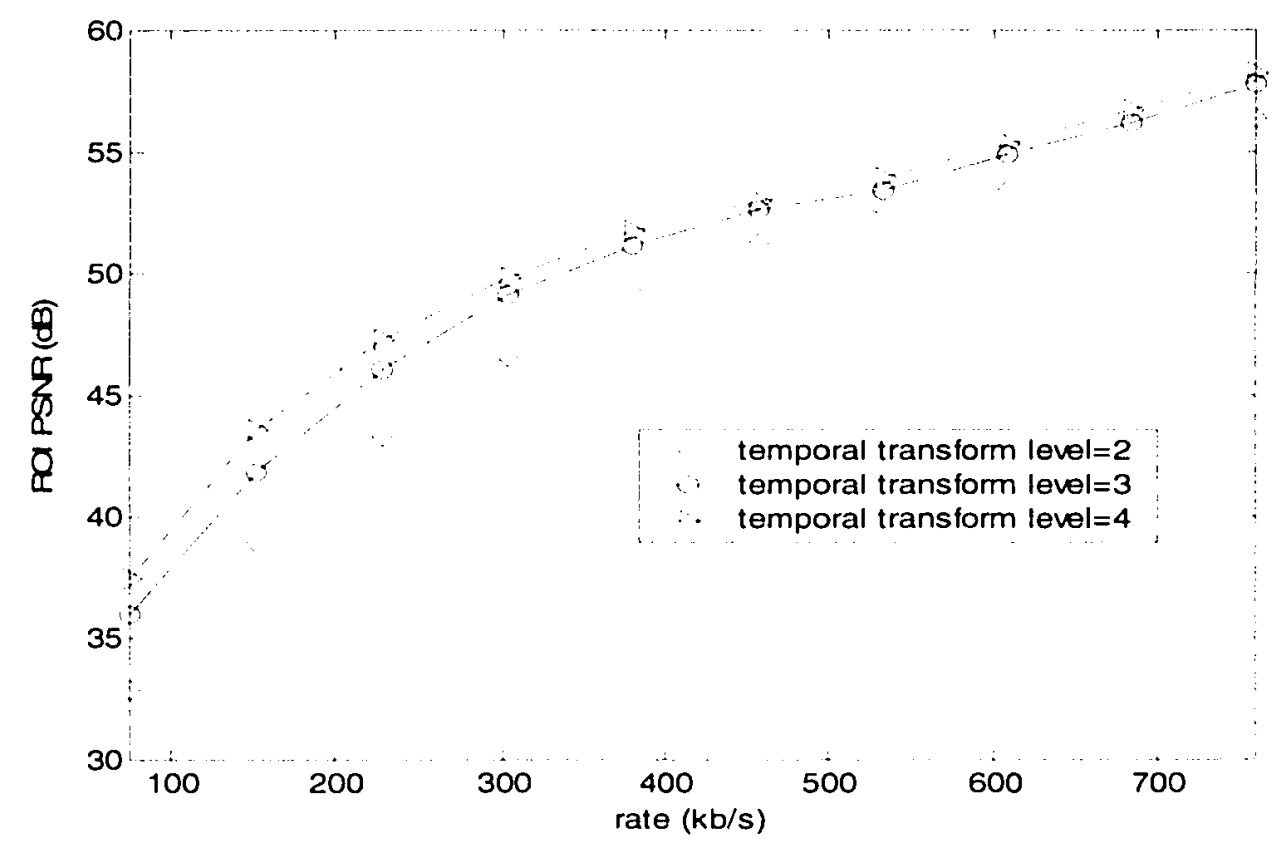

Figure 6.18 Comparison of performance for 'Akiyo' at different temporal transform levels $(\mathrm{GOF}=32)$

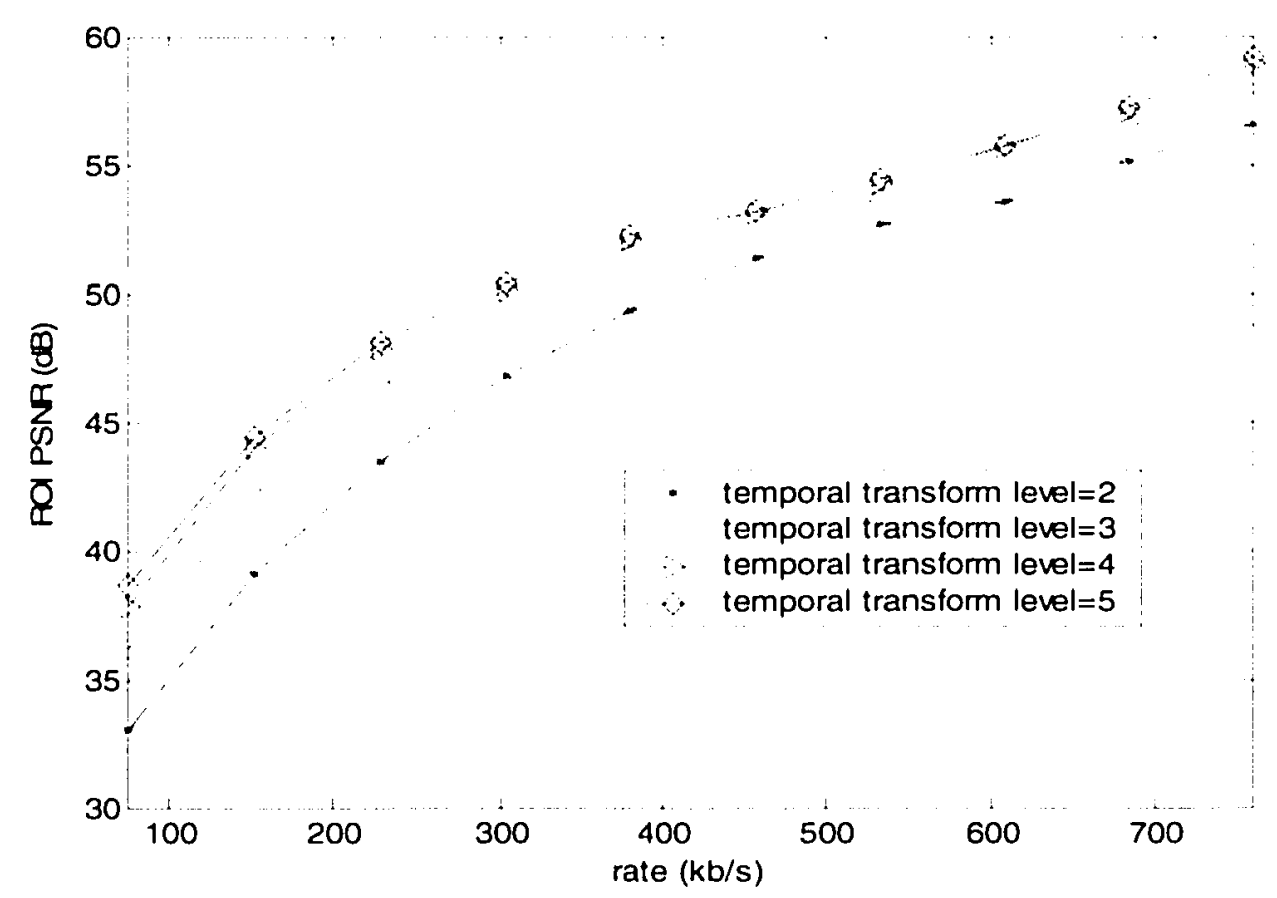

Figure 5.19 Comparison of performance for 'Akiyo' at different temporal transform levels $(\mathrm{GOF}=64)$ 
We also used QCIF 'carphone' video sequence at $30 \mathrm{fps}$ to compare our algorithm with the other algorithms. In this case, the GOF size is 16 and 3 transform levels are used in all dimensions. Figure 6.18 illustrates the performance of UT-SPIHT algorithm is still better than other compared algorithms.

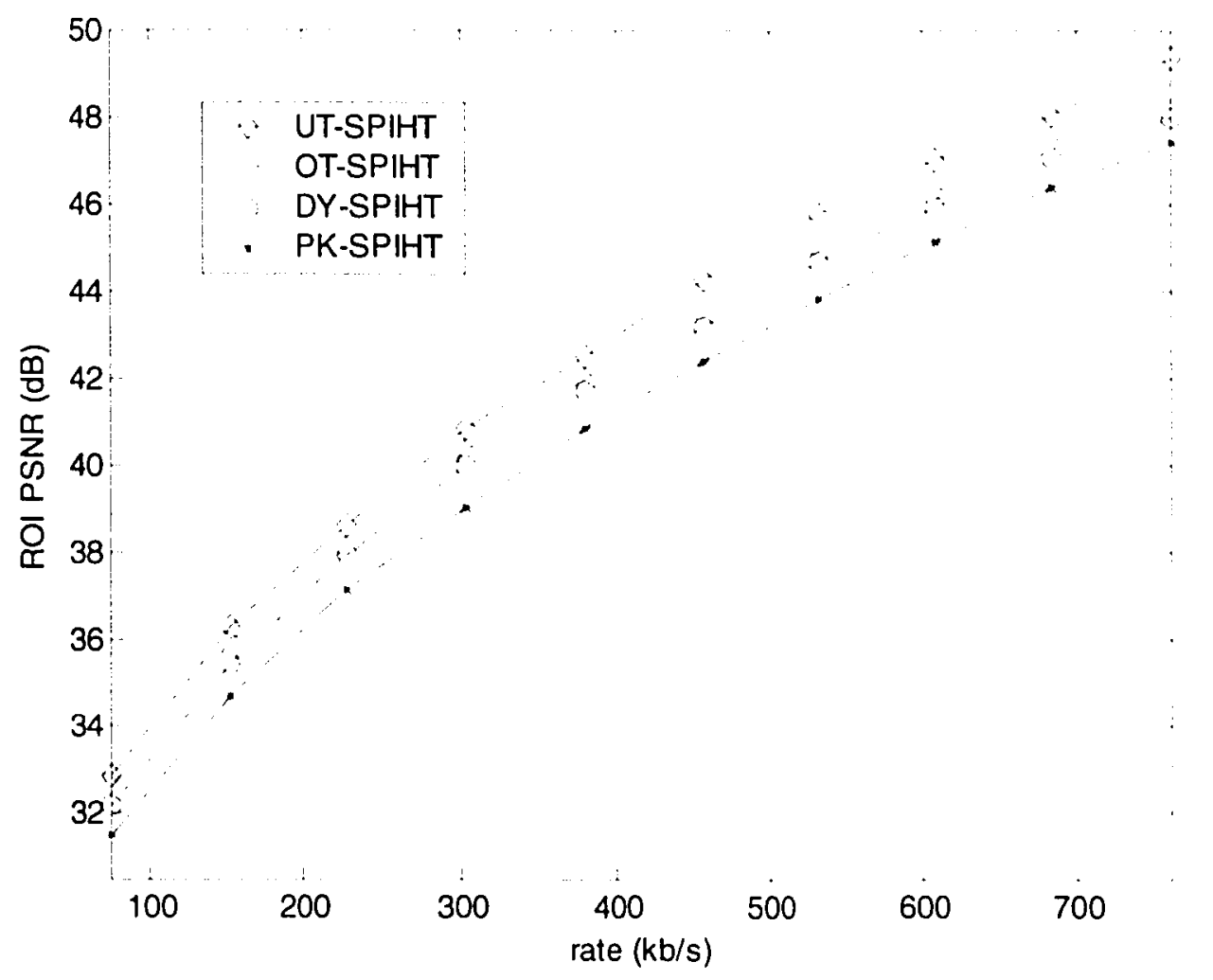

Figure 6.20 Rate-distortion performance for 'Carphone' video using different algorithms As to the performance of MRMQ-SPIHT algorithm on video coding, the results are similar to those in the case of volumetric datasets. We compare the MRMQ-SPIHT with conventional dyadic 3D SPIHT algorithm on 'Akiyo' video with GOF of 64 using 3 transform levels along all directions. The results are shown in Figure 6.19. On average 
UT-SPIHT achieves about $5 \mathrm{~dB}$ improvement over conventional dyadic 3D SPIHT. Example figures of reconstructed video frames are also in Figure 6.20. Figure 6.21 give the reconstructed frames in the case of applying MRMQ-SPIHT on 'Carphone' video sequence with GOF 16.

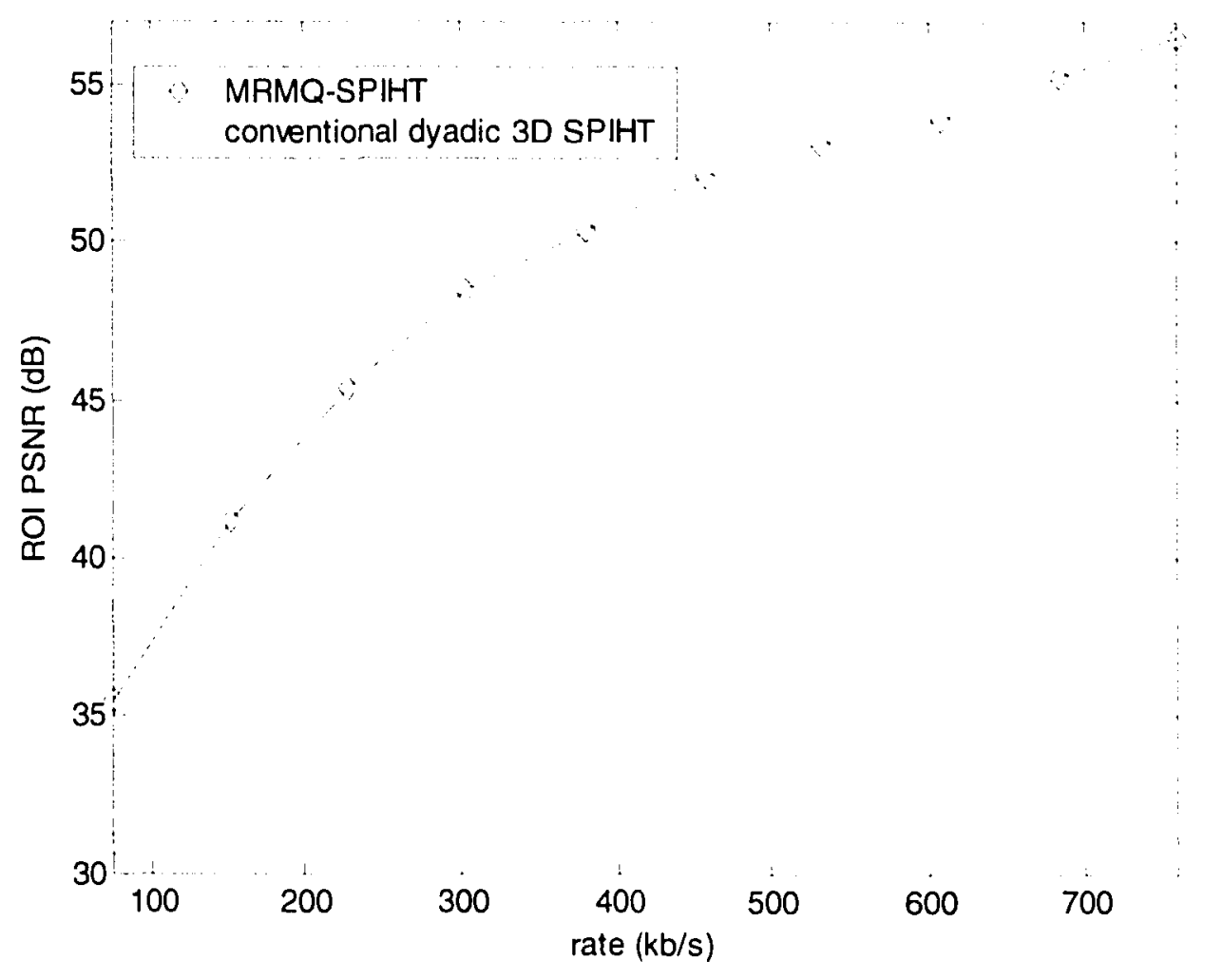

Figure 6.21 Comparison of ROI PSNR by MRMQ-SPIHT and conventional dyadic 3D SPIHT on Akiyo video 

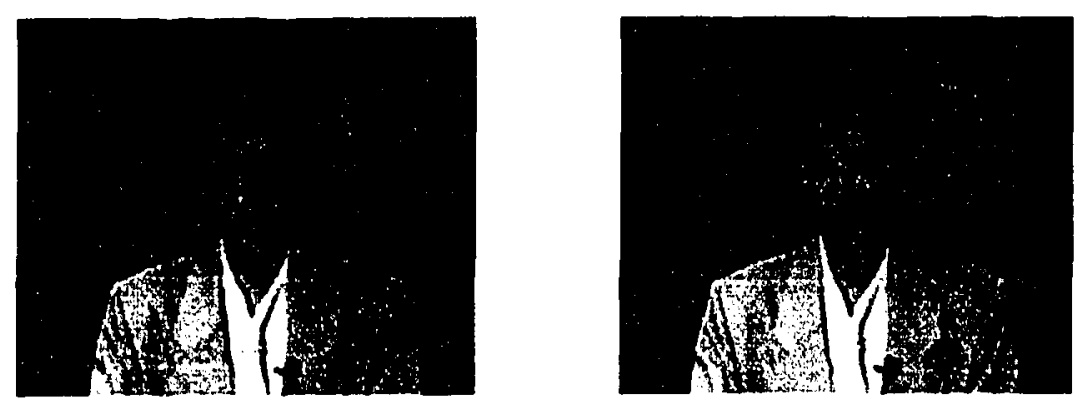

Figure 6.22 Reconstructed "Akiyo" frames of number 10 and 20 at rate $228.096 \mathrm{~kb} / \mathrm{s}$ with $90 \%$ bits for ROI and 10\% bits for background
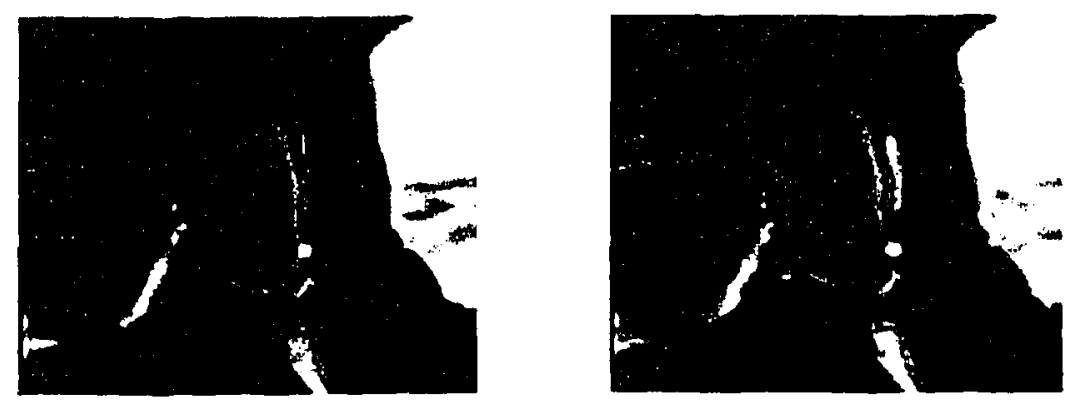

Figure 6.23 Reconstructed "Carphone" frames of number 5 and 15 at rate $228.096 \mathrm{~kb} / \mathrm{s}$ with $90 \%$ bits for ROI and 10\% bits for background

\subsection{Summary}

1. OT-SPIHT and UT-SPIHT exhibit better R-D performance than DY-SPIHT and PKSPIHT on both volumetric medical datasets and video sequences. UT-SPIHT achieves the best coding performance among all compared algorithms. The reason is that the UT-SPIHT algorithm adopts optimal tree as base tree structure to construct the unbalanced spatio-temporal (interslice) orientation tree, which creates longer coefficient trees and exploits the dependency of subbands in packet decomposition

2. The proposed UT-SPIHT algorithm shows promise in ROI-based coding. It exhibits 
better R-D performance compared to state-of-the-art compression algorithms. It avoids the heterogeneity of coefficient trees by reorganizing the parent and children coefficients that belong to different regions. Therefore, coding bits for coefficients unrelated to the ROI are not required as in conventional zerotree structure.

3. Another advantage is that it offers multiple ROI coding with different qualities. By using the separate transform representations of ROIs and region labels to identify coefficients inside the ROIs or outside the ROIs, MRMQ-SPIHT algorithm implements multiple ROI coding. For medical applications, where multiple regions of interest determined as of diagnostic significance, or the video sequence having multiple objects, this coding method can encode these regions separately at different qualities based on the requirement. 


\section{Chapter 7}

\section{Conclusions}

\subsection{Summary}

Large sets of images generated by current medical imaging technologies, such as MRI and $\mathrm{CT}$, require an efficient image compression scheme to be used to reduce cost of image storage and transmission time in the applications, such as PACS and telemedicine. Furthermore, there is a growing demand for efficient compression of video data for applications such as video streaming over internet and video database browsing. Conventional video compression using motion compensation and discrete cosine transform (DCT), which offers the advantages of reasonably high compression ratio and availability of standards for implementation does not support the features required by today's applications including low computational complexity, scalability and good ratedistortion performance. To satisfy these requirements for compression of volumetric datasets and video sequences, a compression method combining three-dimensional wavelet transform and a tree coding algorithm has been studied to take advantage of the dependencies of data in both spatial and temporal/interslice dimensions. 
In some applications, only certain regions in the volumetric data or video are of interest. For example, in MR image of the skull, the brain region can be of particular interest to the physician for diagnostic purposes. In a videoconferencing application, the speaker's head and shoulders are of main interest and need to be coded at higher quality whereas the background can be either discarded or encoded at a much lower rate. High compression ratios can be achicved by allocating more bit rate for region(s) of interest and less bit rate for the remaining regions. Region-based image coding schemes using heterogeneous (multiple) quality constraints are especially attractive because they not only can well preserve the diagnostic features in region(s) of interest, but also meet the requirements of less storage and shorter transmission time for medical imaging applications and multimedia systems.

Shape-adaptive discrete wavelet transform (SA-DWT) with symmetric odd length biorthogonal wavelet basis is used to decompose each ROI independently. SA-DWT offers important features, such as preserving spatial correlation, locality and selfsimilarity across subbands, which facilitate effective coding of coefficients belonging to the ROI. In 3D case, the proposed multiple region decomposition strategy creates a partitioned wavelet coefficient cube where coefficients associated to different regions are completely separated. The extended unbalanced tree structure in three dimensions is employed to establish the homogeneous coefficient trees. The UT-SPIHT is proposed to compress the volumetric images with one ROI; and MRMQ-SPIHT coding algorithm is used to encode the multiple partitioned coefficient cubes by allocating a portion of the 
total bit budget for each region. The results on volumetric datasets and video sequences show that the proposed algorithms provides better R-D performance to that of recently proposed 3D ROI coders.

The most important advantage of MRMQ-SPIHT coding is that it is flexible in encoding coefficients of different regions at different fidelities altogether, which enables a distortion scalable bit stream with efficient ordering of contributions of different regions. Experimental results verify the strength of the proposed tree structure when 3D image data are partitioned into multiple regions. UT-SPIHT obtained better coding performance than other ROI coding algorithms in different settings both on volumetric medical images and video sequence. 


\subsection{Future Work}

- In this thesis, we were focused on lossy coding of volumetric datasets and video sequences. Lossless ROI coding of 3D medical data or video is a future research topic. Three-dimensional integer wavelet transforms using lifting map integer pixels to integer coefficients so that lossless coding can be achieved without information loss. Therefore, combining with 3D integer wavelet transform and SA-DWT, the proposed MRMQ algorithm can be used to compress ROIs from lossy to lossless.

- 3D compression algorithms currently presented in the literature represent each GOS (GOF) of volumetric image data or video in the form of a single bit stream. When a single slice or some slices are needed, the whole volume of image slices has to be decoded from the encoded bit stream. A future research direction is to decode 2-D image data through 3-D encoded bit stream, to provide random accessibility [36] [37]. Combination of random accessibility and MRMQ coding technologies can be useful features in various imaging applications.

- 3D SPIHT coding algorithm without motion compensation provides good coding performance compared to existing video coding standard, such as MPEG-2 [4] with the added features of scalability and simplicity. In video compression, due to the motion of object in the sequence of frames, motion compensation can be considered as an option in coding algorithm to improve compression performance although motion compensation will increase the complexity of algorithm and amount of 
computation.

102

Reproduced with permission of the copyright owner. Further reproduction prohibited without permission. 


\section{Bibliography}

[1] S. Tasdoken and A. Cuhadar, "ROI Coding with Integer Wavelet Transforms and Unbalanced Spatial Orientation Trees", Proceedings of the $25^{\text {th }}$ Annual International Conference of IEEE EMBS, vol. 1, pp. 841-844, September 17-21, 2003.

[2] Ali Bilgin, G. Zweig, and M. W. Marcellin, "Three-dimensional image compression with integer wavelet transforms", Applied Optics, vol. 39, pp. 1799-1814, April 2000

[3] S. Li and W. Li, "Shape-Adaptive Discrete Wavelet Transforms for Arbitrarily Shaped Visual Object Coding", IEEE Trans. On Circuits and Systems for Video Technology, vol. 10, no. 5, pp. 725-743, Aug. 2000.

[4] Z. Xiong, X.Wu, S. Cheng and J. Hua, "Lossy-to-Lossless Compression of Medical Volumetric Data Using Three dimensional Integer Wavelet Transforms," IEEE Trans. On Medical Imaging, vol. 22, No. 3, pp. 459-470, March 2003

[5] P. Schelkens, A. Munteanu, J. Barbarien, M. Galca, X. Giro-Nieto, J. Cornelis, "Wavelet Coding of Volumetric Medical Datasets", IEEE Trans. On Medical Imaging, vol. 22, no. 3, pp. 441-458, March 2003

[6] C. He, J. Dong, Y. F. Zheng, Z. Gao, "Optimal 3-D Coefficient Tree Structure for 3D Wavelet Video Coding", IEEE Trans. On Circuits and Systems for Video Technology, vol. 13, no. 10, pp. 961-972, October 2003 
[7] G. Ginesu, D. Giusto, W. A. Pearlman, "Lossy to Lossless SPIHT-based Volumetric Image Compression", Proceedings of IEEE International Conference on Acoustics, Speech, and Signal Processing, vol. 3, 17-21, pp. 693-696, May 2004

[8] D. Agrafiotis, D. R. Bull and N. Canagarajah, "Region of Interest Coding of Volumetric Medical Images", Proceedings of International Conference on Image Processing, vol. 3, 14-17, pp. 217-220, Sept. 2003

[9] B. Kim, Z. Xiong, and W. A. Pearlman, "Low Bit-rate Scalable Video Coding with 3-D SPIHT", IEEE Trans. On Circuits and Systems for Video Technology, vol. 10, no. 8, pp. 1374-1387, December 2000

[10] G. Minami, Z. Xiong, A. Wang, and S. Mehrotra, "3-D Wavelet Coding of Video with Arbitrary Regions of Support", IEEE Trans. On Circuits and Systems for Video Technology, vol. 11, no. 9, pp. 1063-1068, September 2001

[11] Yun Q. Shi, Huifang Sun, "Image and Video Compression for Multimedia Engineering, Fundamentals, Algorithms, and Standards", CRC Press, 2000

[12] R.J. Clarke, "Digital compression of still images and video", Academic Press, 1995

[13] David S. Taubman, Michael W. Marcellin, "JPEG2000: image compression fundamentals, standards and practice", Kluwer Academic Publishers, 2002

[14] David S. Taubman, "High Performance Scalable Image Compression with EBCOT", IEEE Trans. On Image Processing, vol. 9, no. 7, pp. 1158-1170, July 2000

[15] Mohammed Ghanbari, "Standard Codecs: Image Compression to Advanced Video Coding", The Institution of Electrical Engineers, 2003 
[16] Khalid Sayood, "Introduction to Data Compression", Second Edition, Morgan Kaufmann, 2000

[17] M. Vetterli, J. Kovacevic, "Wavelets and Subband Coding", Prentice Hall, 1995

[18] Lokenath Debnath, "Wavelet transforms and time-frequency signal analysis", Birkhäuser, 2001

[19] R.M. Rao and A. S. Bopardikar. "Wavelet transforms: introduction to theory and applications", Addison-Wesley, 1998

[20] L. Prasad, S.S. Iyengar, "Wavelet analysis with applications to image processing", CRC Press, 1997

[21] J. C. Goswami, A. K. Chan, "Fundamentals of Wavelets, theory, algorithms, and applications", Wiley Inter-science, 1999

[22] Randy K. Young. "Wavelet theory and its applications", Kluwer Academic Publishers, 1993

[23] M. Penedo, W. A. Pearlman "Region-Based Wavelet Coding methods for Digital Mammography", IEEE Trans. on Medical Imaging, vol. 22, no. 10, pp. 1288-1296, October 2003

[24] A. Said and W. A. Pearlman, "A New, Fast, and Efficient Image Codec Based on Set Partitioning in Hierarchical Trees", IEEE Trans. On Circuits and Systems for Video Technology, vol. 6, no. 3, pp. 24.3-250, June 1996

[25] Sinan Tasdoken, "Multiple-Region Multiple-Quality Coding Schemes for Medical Image Compression", Master Thesis, 2002 
[26] Zh. Liu, J. Hua, Z. Xiong, "Lossy-to-Lossless ROI coding chromosome images using modified SPIHT and EBCOT", Proceedings of IEEE International Symposium on Biomedical Imaging, 7-10, pp. 317-320, July 2002

[27] K. Park, and H. Park, "Region-of-Interest Coding Based on SPIHT", IEEE Trans. On Circuits and Systems for Video Technology, vol. 12, no. 2, pp. 106-113, February 2002

[28] L. Liu, and G. Fan, "A New JPEG2000 Region-of-Interest Image Coding Method: Partial Significant Bitplanes Shift”, IEEE Signal Processing Letters, vol. 10, no. 2 , pp. 35-38, February 2003

[29] I. Ueno, and W. A. Pearlman, "Region-of-Interest Coding in volumetric images with shape-adaptive wavelet transform". Proceedings of SPIE, pp. 1048-1055, May 2003

[30] G. Menegaz, and J. Thiran, "Lossy to Lossless Object-Based Coding of 3-D MRI Datta", IEEE Trans. on Image Processing, vol. 11, no. 9, pp. 1053-1061, September 2002

[31] P. Schelkens, C Brislawn, J Barbarien, A Munteanu, "JPEG2000-Part 10: Volumetric Imaging", Proceedings of SPIE, pp. 296-305, Aug. 2003

[32] S. Cho, W. A. Pearlman, "Region-Based SPIHT Coding and Multiresolution Decoding of Image Sequences". Picture Coding Symposium (PCS-2001), Seoul, Korea, 25-27 pp. 283-286, April, 2001 
[33] M. Antonini, M. Barlaud, P. Mathieu, and I. Daubechies, "Image coding using wavelet transform”, IEEE Trans. Image Processing, vol. 1, pp. 205-220, Apr. 1992

[34] B. Kim and W. A. Pearlman. (1998, Marr) Fast color-embedded video coding using SPIHT. [online]. Available: http://www.cipr.rpi.edu/ pealman/

[35] J. Xu, Z. Xiong, S. Li and Y. Zhang, "Memory-Constrained 3D Wavelet Transform for Video Coding Without Boundary Effects", IEEE Trans. on Circuits and Systems for Video Technology, vol. 12, no. 9, pp. 812-818, SEPT. 2002

[36] G. Menegaz, J. Thiran, "Three-Dimensional Encoding/Two-Dimensional Decoding of Medical Data”, IEEE Trans. On Medical Imaging, vol. 22, no. 3, pp. 424-440. March 2003

[37] R. Leung, D. Taubman, "Transform and Embedded Coding Techniques for Maximum Efficiency and Random Accessibility in 3D Scalable Compression", [online]. Available: http://www.ec.unsw.edu.au/ Laubman/publications_files/3dmodeling-access-tip2004.pdf 